\title{
ESTUDO DE MÁXIMO CARREGAMENTO EM SISTEMAS DE ENERGIA ELÉTRICA
}

\section{Cristiano da Silva Silveira}

Dissertação apresentada à Escola de Engenharia de São Carlos, da Universidade de São Paulo, como parte dos requisitos para obtenção do título de Mestre em Engenharia Elétrica.

ORIENTADOR: Prof. Dr. Geraldo R. M. da Costa

São Carlos

2003 
“Se você abre uma porta, você pode ou não entrar em uma nova sala.

Você pode não entrar e ficar observando a vida.

Mas se você vence a dúvida, o temor, e entra, dá um grande passo: nesta sala vive-se! Mas, também, tem um preço... São inúmeras outras portas que você descobre. Às vezes curte-se mil e uma.

O grande segredo é saber quando e qual porta deve ser aberta.

$A$ vida não é rigorosa, ela propicia erros e acertos.

Os erros podem ser transformados em acertos quando com eles se aprende.

Não existe a segurança do acerto eterno.

A vida é generosa, a cada sala que se vive, descobrem-se tantas outras portas.

$E$ a vida enriquece quem se arrisca a abrir novas portas.

Ela privilegia quem descobre seus segredos e, generosamente, oferece afortunadas portas. Mas a vida também pode ser dura e severa.

Se você não ultrapassar a porta, terá sempre a mesma porta pela frente.

É a repetição perante a criação, é a monotonia monocromática perante a multiplicidade das cores, é a estagnação da vida...

Para a vida, as portas não são obstáculos, mas diferentes passagens!"” 
Deus está em tudo, e devemos buscá-lo onde Ele está mais próximo...

...dentro de nós. 
Aos meus pais, Acyr e Maria Apparecida.

Pelo amor, compreensão e apoio. 


\section{AGRADECIMENTOS}

Ao Professor Dr. Geraldo Roberto Martins da Costa, pela orientação, participação e, sobretudo, companheirismo durante a elaboração deste trabalho.

Aos professores e funcionários do Departamento de Engenharia Elétrica da Escola de Engenharia de São Carlos, pela atenção e colaboração.

A todos os colegas da pós-graduação, pelo apoio e amizade.

À minha namorada Giovana, pelo carinho, compreensão e presença nos momentos mais difíceis.

Aos amigos do LOSEP: Alessandra, Edmarcio, Thales e Vanusa, pela acolhida, troca de idéias e, principalmente, pela amizade.

À CAPES - Coordenadoria de Aperfeiçoamento de Pessoal de Nível Superior, pelo apoio financeiro concedido durante a realização deste trabalho.

A TODOS que estiveram ao meu lado durante mais esta etapa da minha vida, muito obrigado! Vocês foram fundamentais! 


\section{SUMÁRIO}

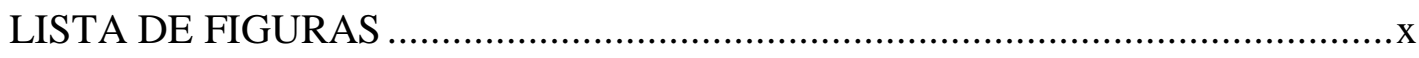

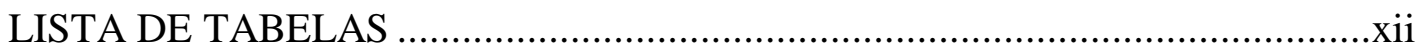

LISTA DE ABREVIATURAS E SIGLAS …............................................. xiii

LISTA DE SÍMBOLOS ............................................................................

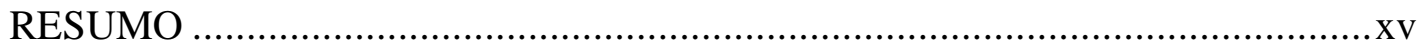

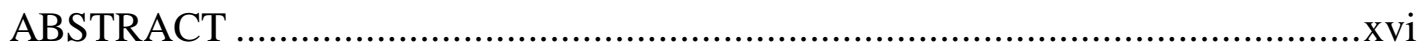

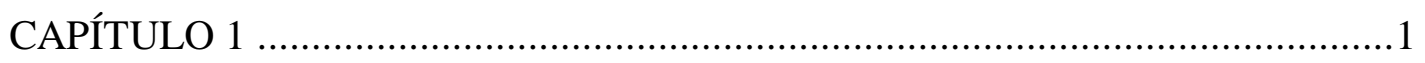

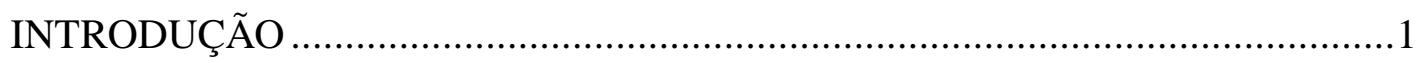

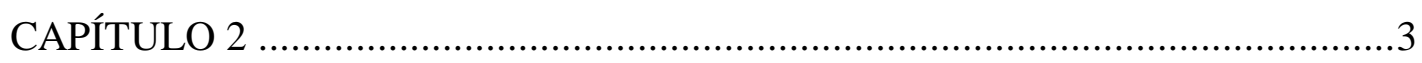

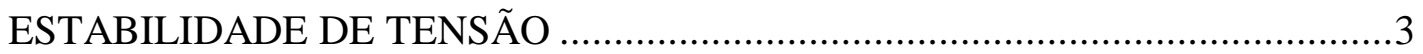

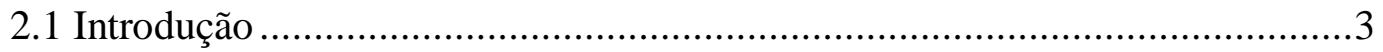

2.2 Definições e Conceitos Básicos ......................................................................3

$2.3 \mathrm{O}$ que se deseja em um Estudo de Estabilidade de Tensão?............................5

2.4 Análise Estática versus Análise Dinâmica ..................................................5

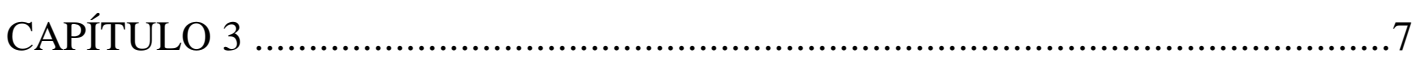

ALGUNS ASPECTOS TEÓRICOS RELEVANTES .........................................

3.1 Transmissão de Potência Reativa ..........................................................

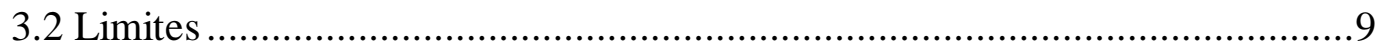

3.3 Manutenção dos Níveis Viáveis de Tensão ................................................ 11

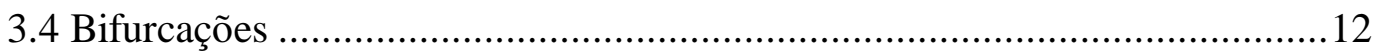


3.4.1 Bifurcações Sela-Nó e o Colapso de Tensão ......................................... 12

3.4.1.1 Bifurcações Sela-Nó das Soluções de uma Equação Quadrática ......12

3.4.1.2 Equilíbrios Estável e Instável ...................................................... 14

3.4.1.3 Autovalores no Ponto de Bifurcação Sela-Nó.................................. 15

3.4.1.4 Diagramas de Bifurcação .............................................................. 16

3.5 Modelos de Carga e Características PV da Rede ........................................ 17

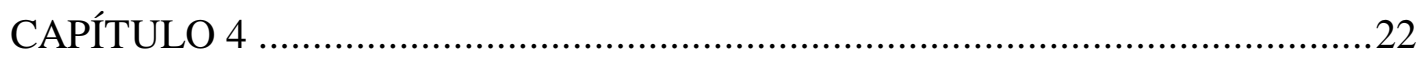

ÍNDICES DE ESTABILIDADE DE TENSÃO ..............................................22

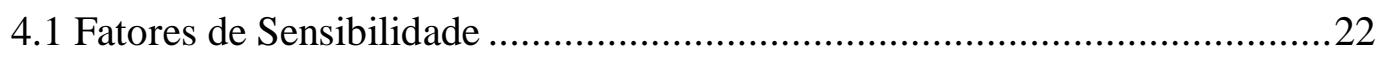

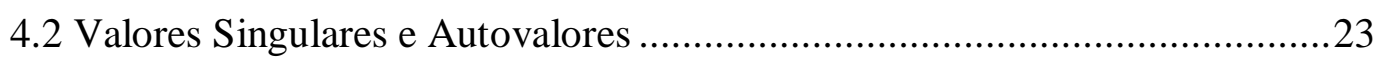

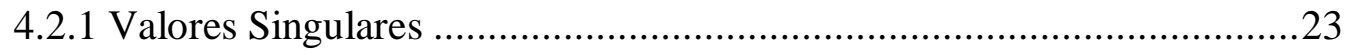

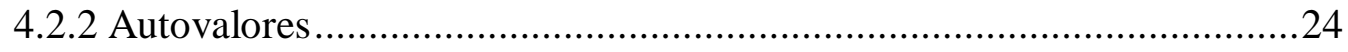

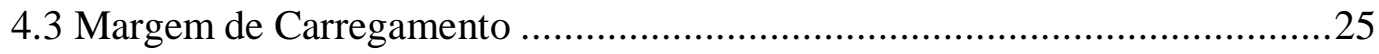

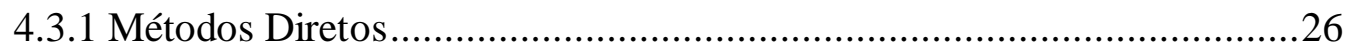

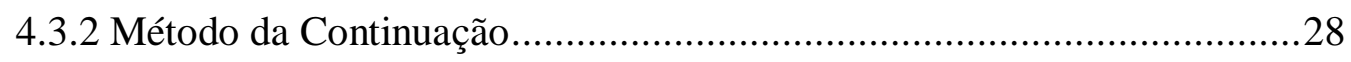

4.3.2.1 Passo Preditor e Parametrização .....................................................29

4.3.2.1.1 Alguns Detalhes sobre Parametrização .................................. 32

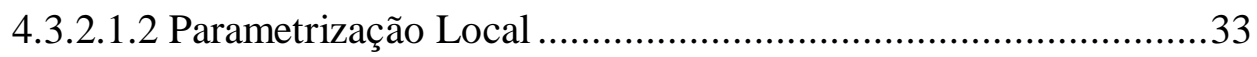

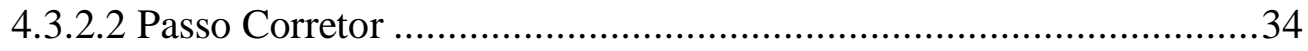

4.4 Par de Soluções do Fluxo de Potência............................................................. 35

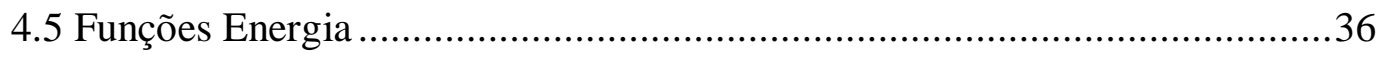

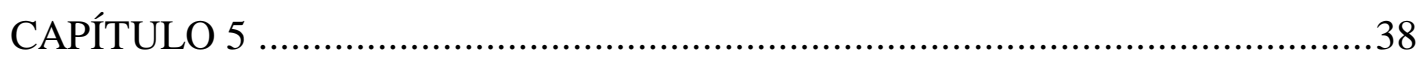

MÉTODO DA CONTINUAÇÃO APLICADO AO PROBLEMA DE FLUXO DE

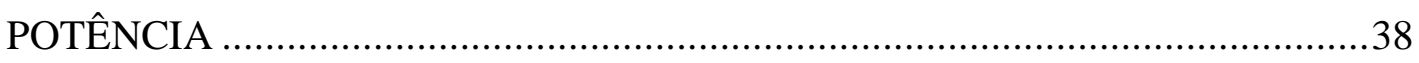

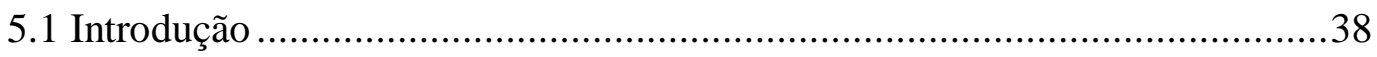

5.2 Reformulação das Equações do Fluxo de Potência....................................... 38

5.3 Aplicação do Método da Continuação ........................................................39 
5.4 Fluxograma do Método da Continuação Aplicado ao Problema de Fluxo de

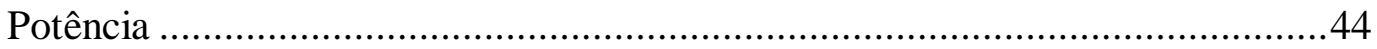

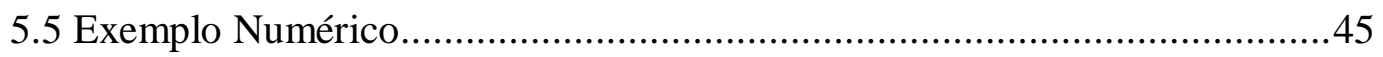

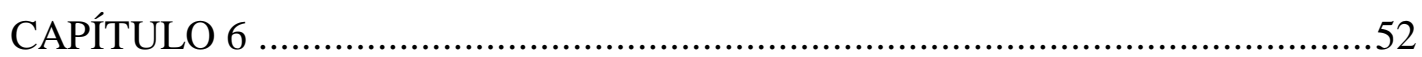

TÉCNICA DE CONTROLE DO TAMANHO DO PASSO ...................................52

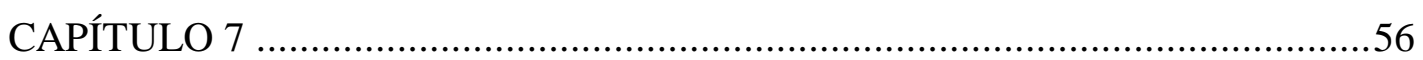

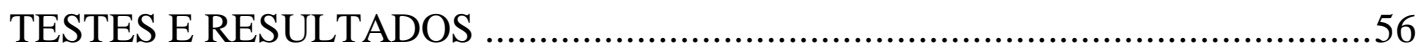

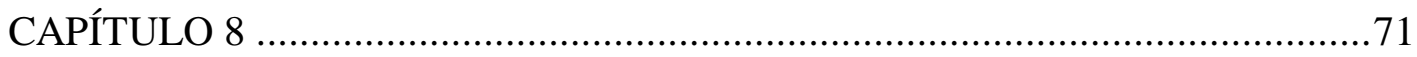

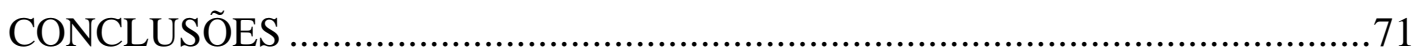

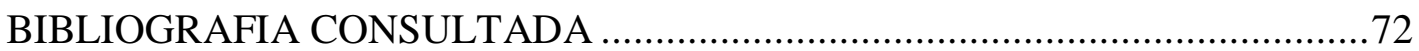

APÊNDICE

GLOSSÁRIO 


\section{LISTA DE FIGURAS}

Figura 1 - Modelo simplificado de um sistema elétrico de potência com duas barras7

Figura 2 - Limite de potência reativa atingido resultando em um ponto de operação estável

Figura 3 - Limite de potência reativa atingido resultando em um ponto de operação instável

Figura 4 - Consumo de potência ativa 13

Figura 5 - Variação da magnitude de tensão com o carregamento 13

Figura 6 - Equilíbrios estável e instável para um carregamento moderado. 14

Figura 7 - Equilíbrio instável para um carregamento máximo 15

Figura 8 - Equilíbrio inexistente para um carregamento maior que o máximo 15

Figura 9 - Característica PV para um sistema sem perdas, com fator de potência unitário e sem a ação de dispositivos de controle e proteção

Figura 10 - Família de curvas PV's para diferentes valores de fator de potência.....19

Figura 11 - Pontos de operação possíveis no plano PV ..........................................21

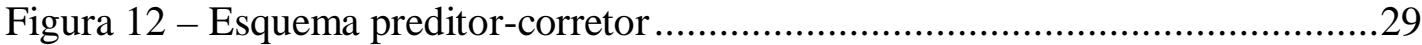

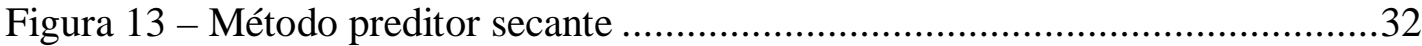

Figura 14 -Problema associado ao método preditor secante ................................32

Figura 15 - Técnica de correção baseada em um vetor perpendicular .....................34

Figura 16 - Técnica de correção baseada em uma parametrização local .................35

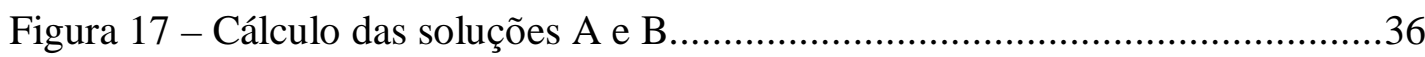

Figura 18 - Representação geométrica das funções energia .................................37

Figura 19 - Método da continuação aplicado às equações do fluxo de potência ......44

Figura 20 - Extremidade da parábola que representa $f(x)$..................................46

Figura 21 - Método da continuação parametrizado por $\lambda$.....................................49

Figura 22 - Método da continuação parametrizado localmente ...............................50

Figura 23 - Trajetória de soluções para diferentes tamanhos de passos..................51

Figura 24 - Controle do tamanho do passo .......................................................54

Figura 25 - Diagrama unifilar de um sistema de três barras ...................................57

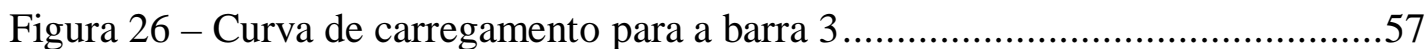




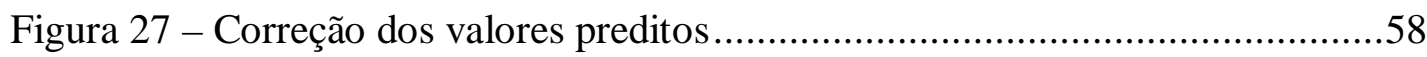

Figura 28 - Comportamento do ângulo de fase da barra 3 durante o carregamento .59

Figura 29 - Curva de carregamento, com e sem limites de reativos, para a barra 14 (IEEE-14 barras) .........................................................................61

Figura 30 - Comportamento do ângulo de fase da barra 14 com e sem limites de reativos (IEEE-14 barras)..............................................................62

Figura 31 - Curva de carregamento, com e sem limites de reativos, para a barra 30

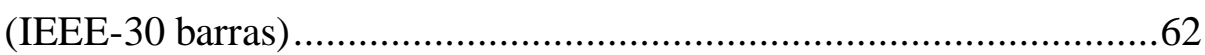

Figura 32 - Comportamento do ângulo de fase da barra 30 com e sem limites de reativos (IEEE-30 barras).

Figura 33 - Curva de carregamento, com e sem limites de reativos, para a barra 31 (IEEE-57 barras). 63

Figura 34 - Comportamento do ângulo de fase da barra 31 com e sem limites de reativos (IEEE-57 barras) .64

Figura 35 - Curva de carregamento, com e sem limites de reativos, para as barras 76 e 44 respectivamente (IEEE-118 barras) .64

Figura 36 - Comportamento do módulo da tensão da barra 76 sem e com limites de reativos (IEEE-118 barras). .65

Figura 37 - Curva de carregamento para as barras 45 e 81 (IEEE-118 barras) ........65

Figura 38 - Cálculo do PMC via controle de passo (barra 14; IEEE-14 barras) ......66

Figura 39 - Cálculo do PMC via controle de passo (barra 30; IEEE-30 barras) ......67

Figura 40 - Cálculo do PMC via controle de passo (barra 31; IEEE-57 barras) ......68

Figura 41 - Cálculo do PMC via controle de passo (barra 44; IEEE-118 barras).....69 


\section{LISTA DE TABELAS}

Tabela 1 - Características do sistema de 3 barras no PMC ...................................59

Tabela 2 - Características do sistema IEEE-14 barras no PMC ...............................60

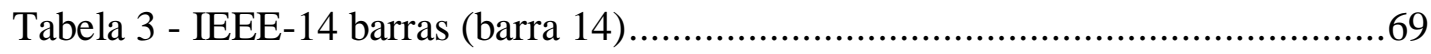

Tabela 4 - IEEE-30 barras (barra 30) .................................................................. 70

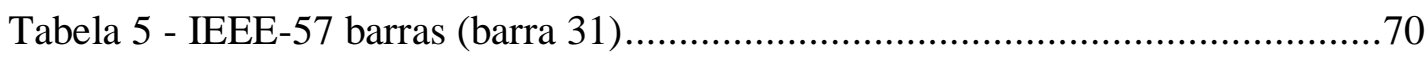

Tabela 6 - IEEE-118 barras (barra 44) ...................................................... 70 


\section{LISTA DE ABREVIATURAS E SIGLAS}

$\mathrm{C}++-$ linguagem de programação

CPU - Central Processing Unit

IEEE - Institute of Electrical and Electronics Engineers

MATLAB - Matrix Laboratory

MW - mega watts

MVAr - mega volt-ampère reativo

PMC - ponto de máximo carregamento

p.u. - por unidade 


\section{LISTA DE SÍMBOLOS}

$\mathrm{V}$ - magnitude de tensão

$\delta$ - ângulo de tensão

$\mathrm{S}$ - potência aparente

$\mathrm{P}$ - potência ativa

$\mathrm{Q}$ - potência reativa

$P Q$ - barra de carga

PV - barra de carga com controle de reativos ou barra de geração

$\mathrm{w}$ - autovetor à direita

$\lambda$ - parâmetro de carga ou parâmetro de carregamento

$\boldsymbol{\omega}$ - multiplicadores de Lagrange

J - matriz Jacobiana

$\sigma$ - tamanho do passo a ser dado na direção do vetor tangente

$\sigma_{0}$ - valor inicial para o tamanho do passo a ser dado na direção do vetor tangente

$\sigma_{1}$ - tamanho do passo baseado na norma do vetor tangente

$\varepsilon$ - tolerância de convergência

at - alta tensão

bt - baixa tensão 


\section{RESUMO}

\section{SILVEIRA, C. S.. Estudo de máximo carregamento em sistemas de energia} elétrica. 2003. 76 p. Dissertação Mestrado - Escola de Engenharia de São Carlos, Universidade de São Paulo, São Carlos.

Este trabalho apresenta um estudo sobre o método da continuação aplicado ao problema de fluxo de potência. Definições e conceitos de estabilidade de tensão são descritos de forma a explicitar as diferenças e semelhanças existentes com relação ao estudo de máximo carregamento em sistemas de energia elétrica. Uma síntese da teoria da bifurcação aborda sua importância em estudos de colapso de tensão. É proposta uma técnica de controle do tamanho do passo para o método da continuação com o objetivo de determinar o ponto de máximo carregamento (PMC) sem a necessidade de especificar, por meio do usuário, um valor para o tamanho inicial do passo. Os resultados dos estudos realizados em sistemas testes do IEEE (14, 30, 57 e 118 barras) mostram a aplicação do método da continuação convencional e de sua associação à técnica de controle do tamanho do passo.

Palavras-chave: método da continuação, fluxo de potência, sistemas de energia elétrica, máximo carregamento, teoria da bifurcação, estabilidade de tensão, tamanho do passo. 


\section{ABSTRACT}

SILVEIRA, C. S. (2003). A study about maximum loading in electrical power systems. São Carlos, 2003. 76p. Dissertação (Mestrado) - Escola de Engenharia de São Carlos, Universidade de São Paulo.

This work presents a research about the continuation method applied to the power flow problem. Voltage stability definitions and concepts are described in a way to highlight and point out the differences and the similarities among several methods used to determine the maximum loading of electrical power systems. A short description of the bifurcation theory is also presented in order to show its importance to the voltage collapse studies. A technique based on automatically controlling the step size is proposed as an innovation of the continuation method. The objective of this technique is to determine the maximum loading point without the traditional need of asking the user for the initial step size. The results compare the performance between the conventional and the new method. These methods are analyzed using IEEE test systems (14, 30, 57 and 118-bus).

Key-words: continuation method, power flow, electrical power systems, maximum loading, bifurcation theory, voltage stability, step size. 


\section{CAPÍTULO 1}

\section{INTRODUÇÃO}

$\mathrm{O}$ crescente aumento da demanda dos sistemas de energia elétrica associado à falta de investimentos na expansão e manutenção do setor, tem elevado as dificuldades em atender as cargas deixando a operação do sistema próxima de seus limites de carregamento. Restrições ecológicas e/ou econômicas e a tendência de melhoria da utilização dos recursos e equipamentos dos sistemas de potência também contribuem, de forma decisiva, na consolidação deste cenário. Além do mais, com o advento de um ambiente desregulamentado, a malha de transmissão, interconectada e extremamente complexa, passa a ser utilizada para fins aos quais não foi, a princípio, projetada. Surgem, portanto, dificuldades no seu monitoramento e controle, uma vez que parte da potência produzida pelas empresas geradoras é vendida a outras empresas responsáveis por sua transmissão e distribuição.

Este acréscimo de carga dos sistemas gera um aumento das perdas reativas nas linhas de transmissão. Variações dos módulos das tensões entre os terminais de uma linha de transmissão aliadas a grandes diferenças angulares são responsáveis por possíveis alterações nas perdas de potência reativa. Estas são supridas, em parte, pela geração de reativos proveniente das barras PV's a partir do controle da tensão nos terminais. Nesta tentativa de manutenção de um perfil adequado de tensão (tensões próximas a 1 p.u.), as reservas de reativos podem se exaurir e provocar a perda de controle da tensão por parte dos geradores. Como conseqüência, tem-se uma queda de tensão que ocasiona um aumento na diferença angular entre os terminais da linha de transmissão. $\mathrm{O}$ intuito deste aumento é manter a transmissão de potência ativa. Logo, o aumento da diferença angular somado à queda de tensão e a uma redução imposta do suporte de reativos por parte dos bancos de capacitores causará um novo aumento nas perdas reativas das linhas de transmissão levando a operação do sistema a uma região na qual não há mais o controle de tensão (ALVES, 2000). 
Esta queda gradual das magnitudes de tensão é o estágio inicial de um colapso. Em situações de um decaimento rápido da tensão em sistemas carregados, as ações dos controles ou dos operadores pouco ou nada podem fazer para preveni-lo.

Este trabalho tem como objetivo gerar conhecimentos a respeito da importância do estudo do máximo carregamento e, assim, fornecer condições para o entendimento do problema em si e das técnicas utilizadas em sua análise.

Inicialmente, o Capítulo 2 deste texto apresenta definições e conceitos de estabilidade de tensão, bem como a necessidade de sua análise e as linhas de estudos seguidas para sua compreensão.

O Capítulo 3 descreve características importantes com relação aos sistemas de potência. A consideração ou não destas características interfere significativamente nos resultados. Um outro aspecto abordado diz respeito à ligação entre o fenômeno de colapso de tensão e a teoria da bifurcação.

No Capítulo 4, diversos índices de estabilidade de tensão utilizados para medir a proximidade do colapso de tensão são descritos. Dentre estes índices, aquele de maior interesse é o obtido a partir do método da continuação, enfocado neste trabalho.

O Capítulo 5 apresenta a metodologia alvo do estudo e sua aplicação em um exemplo numérico com o intuito de validá-la.

No Capítulo 6, apresenta-se uma técnica desenvolvida para o controle do tamanho e direção do passo.

O Capítulo 7 mostra resultados da aplicação do método da continuação convencional e de sua associação à técnica de controle do tamanho do passo. São utilizados os sistemas testes do $\operatorname{IEEE}(14,30,57$ e 118 barras).

E, finalmente, o Capítulo 8 apresenta as conclusões deste trabalho de mestrado. 


\section{CAPÍTULO 2}

\section{ESTABILIDADE DE TENSÃO}

\subsection{Introdução}

A possibilidade de ocorrência de problemas ligados à estabilidade de tensão vem se tornando, na atualidade, um assunto de grande preocupação nas empresas de energia elétrica do mundo inteiro. Os fenômenos de estabilidade de tensão estão intrinsecamente ligados ao fluxo de potência reativa sobre a rede, ao comportamento das cargas face às variações de tensão, à ação de dispositivos automáticos de controle de tensão e limitação de sobre-excitação de geradores, entre outros.

A perda de estabilidade de tensão ocorrerá quando houver um declínio progressivo e descontrolado da tensão do sistema após ter sido submetido a um distúrbio, aumento da carga ou mudanças nas suas condições, como a saída de um gerador.

\subsection{Definições e Conceitos Básicos}

Segundo SIMÕES COSTA (2002), os termos instabilidade de tensão e colapso de tensão são freqüentemente usados, indistintamente, para designar o mesmo tipo de fenômeno. A análise do problema se baseia tanto em métodos estáticos (fluxo de potência), quanto em abordagens dinâmicas, que levam em conta a resposta no tempo dos diversos componentes e dispositivos envolvidos. Um dos aspectos fundamentais para a estabilidade de tensão é a capacidade (ou as limitações) de transferir potência reativa das fontes de produção até os centros de consumo. Colapsos de tensão ocorrem, tipicamente, em sistemas de potência extremamente carregados, com escassez de potência reativa e/ou após contingências. Freqüentemente, o colapso de tensão envolve todo o sistema de potência, apesar de áreas particulares deste terem maior envolvimento. As seguintes definições têm sido associadas à estabilidade de tensão: 
"Um sistema de potência operando em uma dada condição de operação e sujeito a uma dada perturbação é estável, do ponto de vista de tensão, se as tensões próximas às cargas assumirem valores de equilíbrio após a perturbação”.

"Um sistema de potência operando em uma dada condição de operação e sujeito a uma dada perturbação sofre um colapso de tensão se as tensões, após a perturbação, estiverem abaixo de limites aceitáveis. Um colapso de tensão pode ser total ou parcial”.

"Um sistema de potência operando em uma dada condição de operação é estável, do ponto de vista de tensão se, para toda barra do sistema, a sua magnitude de tensão aumenta quando a injeção de potência reativa na mesma barra é aumentada; um sistema de potência operando em uma dada condição de operação é instável do ponto de vista de tensão quando, no mínimo, uma barra do sistema tem sua magnitude de tensão reduzida quando a injeção de potência reativa na mesma é aumentada” (KUNDUR et al., 1992).

Em outras palavras:

- Sistema estável $\rightarrow$ sensibilidade V-Q positiva para toda barra;

- Sistema instável $\rightarrow$ sensibilidade V-Q negativa para, no mínimo, uma barra.

Entretanto, uma das definições mais proveitosas é:

“Um ponto de operação de um sistema de potência é estável a um pequeno distúrbio se, seguido de qualquer pequeno distúrbio, o sistema de potência retorna ou fica próximo do mesmo ponto de operação pré-distúrbio" (CAÑIZARES, 2001).

Existem várias alterações nas condições de operação de um sistema de potência que contribuem para o colapso de tensão. A maioria destas alterações tem um efeito significativo na geração, transmissão e consumo de potência reativa: 
- acréscimo no carregamento;

- geradores, condensadores síncronos e controladores estáticos de reativos atingindo seus limites de potência reativa;

- saídas de linhas ou geradores;

- comportamento das cargas face às variações de tensão;

- variação automática de taps de transformadores;

- sistemas de potência estressados, devido à falta de reforço de transmissão e falta de investimentos em geração;

- uso excessivo de bancos de capacitores para compensação de potência reativa que, embora estendam os limites de transferência de potência, tornam o sistema mais inclinado a sofrer problemas de instabilidade de tensão.

O principal fator responsável pela instabilidade de tensão é a incapacidade do sistema de atender à demanda de potência reativa.

\subsection{O que se deseja em um Estudo de Estabilidade de Tensão?}

Existe uma necessidade de ferramentas analíticas capazes de predizer o colapso de tensão em redes complexas quantificando, exatamente, as margens de estabilidade e os limites de transferência de potência; identificando os pontos fracos de tensão do sistema e áreas suscetíveis à instabilidade; e identificando os fatores chave para sua contribuição e sensibilidade que forneçam características do sistema para a adoção de ações corretivas (KUNDUR et al., 1992).

\subsection{Análise Estática versus Análise Dinâmica}

Ocorrências de fenômenos de colapso de tensão foram constatadas em vários países do mundo, e suas razões são as mais variadas possíveis. A análise de cada evento ocorrido no passado pode ser de grande utilidade no entendimento de como o colapso de tensão pode ser identificado e prevenido nos sistemas elétricos de potência. A caracterização de um colapso de tensão como um evento lento ou rápido 
se faz necessária para definir a linha de estudos que será seguida na análise deste fenômeno (ZAMBRONI DE SOUZA, 1995).

Por exemplo, um evento considerado rápido seria a saída de uma linha de transmissão ou de um gerador do sistema. Se a dinâmica do sistema não é "forte" o suficiente para superar esta falta, o sistema perde estabilidade. Logo, a análise deste fenômeno requer um modelo detalhado do sistema, incluindo equações transitórias de geradores. Por outro lado, uma pequena perturbação pode ser a causa da perda da estabilidade do sistema. Nesta situação, a razão do colapso de tensão pode ser alguma linha de transmissão que atinge seu limite máximo de transferência de potência ou a falta de suporte de reativo em alguma barra de geração. Este tipo de colapso de tensão é definido como um problema estático de tensão. Sob estas condições, alguns estudos mostram que as equações do fluxo de potência podem ser usadas para analisar o problema. Tais equações são utilizadas neste trabalho, tendo como alvo o estudo estático do colapso de tensão.

Embora o colapso de tensão seja, essencialmente, um fenômeno dinâmico, sua análise tem sido realizada, intensamente, por meio de métodos estáticos. Isto porque as análises dinâmicas no domínio do tempo, além de consumirem tempos enormes de CPU e de engenharia (antes que as conclusões sejam tiradas a respeito da estabilidade, pode ser necessário monitorar e examinar muitos parâmetros cuidadosamente), não fornecem informações em relação à sensibilidade ou ao grau de estabilidade. Estas limitações tornam o uso da abordagem dinâmica inviável tanto para a monitoração on-line do sistema quanto para a análise de grandes sistemas para os quais se necessita avaliar um grande número de condições e de contingências. Desta forma, os métodos estáticos podem ser usados, efetivamente, para prover uma melhor compreensão do fenômeno como também para a aplicação on-line, objetivando a monitoração, a prevenção e o controle do fenômeno antes da ocorrência de distúrbios que possam levar à instabilidade de tensão e conduzir o sistema para o colapso (ALVES, 2000). 


\section{CAPÍTULO 3}

\section{ALGUNS ASPECTOS TEÓRICOS RELEVANTES}

A eficiência de um estudo de máximo carregamento depende da consideração de alguns conceitos.

\subsection{Transmissão de Potência Reativa}

Para o modelo simplificado de sistema de potência da Figura 1, verifica-se que a potência entregue ao terminal receptor é:

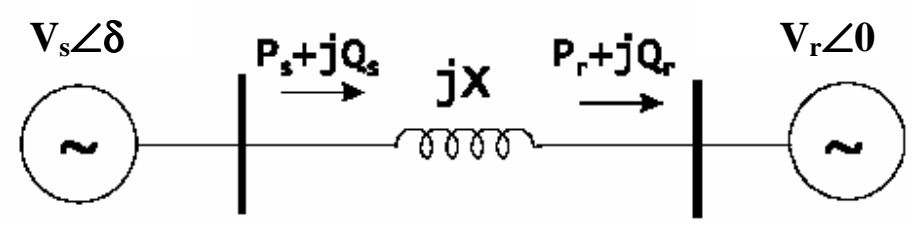

Figura 1 - Modelo simplificado de um sistema elétrico de potência com duas barras

$$
\begin{gathered}
S_{r}=P_{r}+j Q_{r}=\dot{V}_{r} \dot{I}^{*}=\dot{V}_{r}\left(\frac{V_{s} \angle \delta-V_{r}}{j X}\right)^{*} \\
S_{r}=\frac{V_{s} V_{r}}{X} \operatorname{sen} \delta+j\left(\frac{V_{s} V_{r}}{X} \cos \delta-\frac{V_{r}^{2}}{X}\right)
\end{gathered}
$$

Portanto:

$$
\begin{gathered}
P_{r}=\frac{V_{s} V_{r}}{X} \operatorname{sen} \delta \\
Q_{r}=\frac{V_{s} V_{r}}{X} \cos \delta-\frac{V_{r}^{2}}{X}
\end{gathered}
$$

Seguindo-se o mesmo procedimento para o terminal gerador, obtém-se:

$$
\begin{gathered}
P_{s}=\frac{V_{s} V_{r}}{X} \operatorname{sen} \delta \\
Q_{s}=\frac{V_{s}^{2}}{X}-\frac{V_{s} V_{r}}{X} \cos \delta
\end{gathered}
$$


Observa-se que a igualdade entre $P_{s}$ e $P_{r}$ se dá devido a que o sistema simplificado não apresenta perdas $(r=0)$. Para a potência reativa, verifica-se que $Q_{s} \neq Q_{r}$. Os sinais positivos para estas grandezas indicam o sentido representado na Figura 1.

Considerando defasagens pequenas entre $\dot{V}_{s}$ e $\dot{V}_{r}$, tem-se sen $\delta \approx \delta$. Portanto:

$$
P_{r}=P_{s} \approx P_{\max } \delta \text { onde } P_{\max } \stackrel{\Delta}{=} \frac{V_{s} V_{r}}{X},
$$

o que confirma o fato de que a transferência de potência ativa depende, principalmente, da diferença angular entre $\dot{V}_{s}$ e $\dot{V}_{r}$. Para baixos valores de $\delta$ ( $\cos \delta \approx 1$ ), a potência reativa pode ser aproximada por:

$$
\begin{aligned}
& Q_{r} \approx \frac{V_{r}\left(V_{s}-V_{r}\right)}{X} \\
& Q_{s} \approx \frac{V_{r}\left(V_{r}-V_{s}\right)}{X}
\end{aligned}
$$

de onde se conclui que a transmissão de potência reativa depende, principalmente, da magnitude de tensão; e que flui da tensão mais alta para a tensão mais baixa. As observações acima apenas confirmam que:

$$
\begin{aligned}
& P \text { e } \delta \rightarrow \text { são fortemente acoplados, } \\
& Q \text { e } V \rightarrow \text { são fortemente acoplados }
\end{aligned}
$$

Contudo, estas relações falham durante situações em que o sistema é muito exigido. E são estas as relações de interesse, uma vez que os fenômenos de instabilidade de tensão as têm como base.

Uma das razões que limita o transporte de potência reativa sobre a rede é um ângulo de defasagem entre as tensões, $\delta$, elevado. Por exemplo, sejam $\delta=30^{\circ}$; $V_{s}=1,0$ p.u. e $V_{r}=0,9$ p.u.. Logo: 


$$
\begin{gathered}
Q_{s}=\frac{1,0^{2}-1,0 \cdot 0,9 \cdot 0,866}{X}=\frac{0,22}{X}, \\
Q_{r}=\frac{1,0 \cdot 0,9 \cdot 0,866-0,9^{2}}{X}=-\frac{0,03}{X},
\end{gathered}
$$

ou seja, uma grande quantidade de potência reativa é injetada na linha, porém, nada chega à carga. Pelo contrário, a linha solicita $Q=0,03 / X$ do terminal receptor. A linha de transmissão é um "dreno" de potência reativa e consome $0,25 / X$ p.u..

O problema é agravado porque não se pode ter diferenças muito grandes de magnitudes de tensão, já que se exige normalmente que $V=1,0 \pm 0,05$ p.u..

Porém, este não é o único exemplo de limitação de transporte de potência reativa. Do ponto de vista econômico, as perdas de transmissão são uma função direta do quadrado da potência reativa transmitida, e uma função inversa do quadrado da tensão. Portanto, para minimizar perdas deve-se minimizar o transporte de potência reativa sobre as linhas de transmissão, além de manter as tensões elevadas.

Finalmente, deve-se mencionar outra desvantagem do transporte de potência reativa ligado à ocorrência de sobretensões na rede: se a potência reativa transmitida é considerável e um disjuntor próximo à carga se abre, desenvolvem-se sobretensões no lado da carga cujas magnitudes são maiores quanto maior for a potência reativa transmitida. Tais sobretensões são indesejáveis, pois podem colocar em risco a integridade de equipamentos e levar a interrupções no suprimento de energia elétrica (SIMÕES COSTA, 2002).

\subsection{Limites}

Além dos fatores, descritos anteriormente, que limitam o transporte de potência reativa na rede, os limites de potência reativa em geradores e limites de taps em transformadores também causam efeitos significativos em relação ao colapso de tensão. Em geral, o sistema de equações se altera quando estes limites são atingidos. Em alguns casos, o efeito destes limites torna constantes as variáveis de estado do 
sistema. Estas mudanças podem ocasionar diferentes margens de estabilidade, sendo possível atingir um equilíbrio instável, o que causa o colapso de tensão.

O exame de uma curva PV, a ser detalhada no item 3.5, revela como estes limites podem influenciar nas margens de estabilidade de um sistema. É o caso das curvas mostradas na Figura 2, derivadas das equações de um sistema de potência. Em uma destas curvas o limite de potência reativa de um determinado gerador foi atingido, ao passo que na outra, isto não aconteceu. Assume-se que a porção superior de cada curva é estável e que a porção inferior é instável.

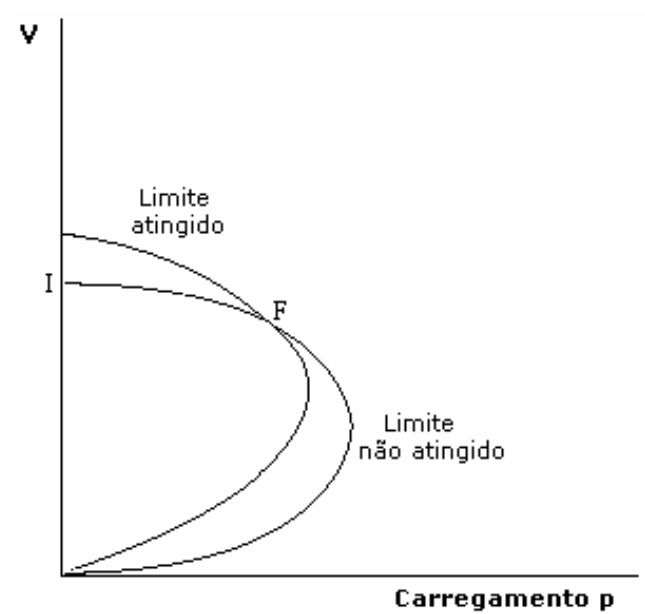

Figura 2 - Limite de potência reativa atingido resultando em um ponto de operação estável

Supõe-se que o sistema esteja operando em um ponto I, distante do limite de potência reativa daquele gerador. Com o acréscimo de carga, os valores das magnitudes das tensões sofrem uma redução. Em contrapartida, a potência reativa gerada aumenta. Em um determinado instante, aquele gerador atinge seu limite de potência reativa no ponto $\mathrm{F}$, alterando as equações do sistema de potência e, consequientemente, o traçado da curva PV. Uma vez que este novo ponto de operação para o sistema se encontra na porção superior da curva, o equilíbrio permanece estável. Porém, como esperado, a margem de estabilidade é reduzida, já que a extremidade da nova curva PV está mais próxima do ponto de operação F.

Na Figura 3, uma situação diferente pode ser observada. Neste caso, quando o limite de potência reativa é atingido, o ponto de operação $\mathrm{F}$ localiza-se na parte inferior da nova curva PV do sistema. Isto significa a existência de um equilíbrio instável, ou seja, tem-se o colapso de tensão. 


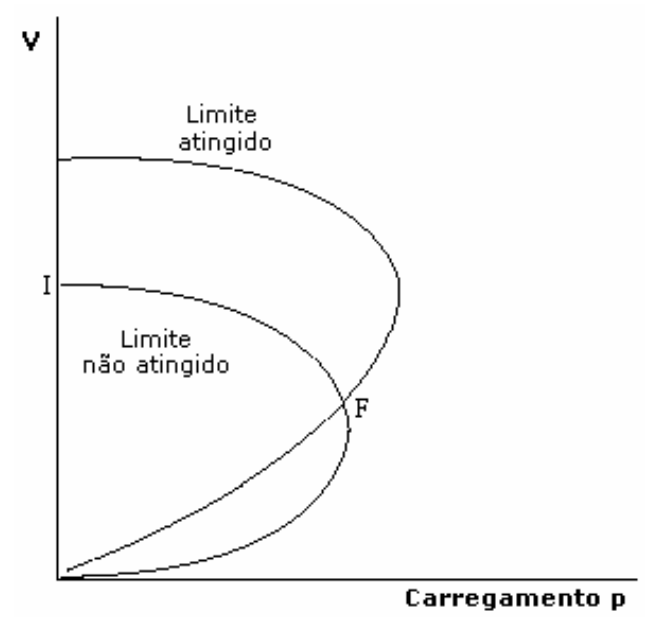

Figura 3 - Limite de potência reativa atingido resultando em um ponto de operação instável

\subsection{Manutenção dos Níveis Viáveis de Tensão}

Os níveis de tensão, regulados dentro de $5 \%$ dos seus valores nominais, são amplamente determinados pelo balanço de geração e consumo de potência reativa. Como as perdas indutivas nas linhas tornam ineficaz a geração de grandes quantidades de potência reativa em linhas longas, grande parte da potência reativa exigida pelas cargas deve ser suprida localmente. Além disso, as limitações de potência reativa que os geradores podem suprir têm uma forte influência nos níveis de tensão e, também, no colapso de tensão. São dispositivos para controle do nível de tensão:

- bancos de capacitores;

- controladores estáticos de reativos;

- transformadores com controle automático de tap sob carga;

- geradores.

O problema de tensão de baixa ou baixa tensão ocorre quando algumas tensões do sistema estão abaixo do limite inferior mas o sistema está operando de forma estável. Desde que um ponto de operação estável persista e nenhum distúrbio aconteça, o problema de baixa tensão pode ser considerado distinto do colapso de tensão. 


\subsection{Bifurcações}

A teoria da bifurcação prediz como um sistema se torna instável. A idéia principal é a de estudar o sistema de potência no limiar da instabilidade (CAÑIZARES, 2001). Há poucos modos pelos quais o sistema se torna instável; independente do tamanho ou da complexidade do modelo do sistema de potência. A teoria da bifurcação descreve esses modos e os respectivos cálculos associados. As análises de bifurcação requerem que o modelo do sistema de potência seja especificado na forma de equações que contenham variáveis de estado e parâmetros.

As bifurcações sela-nó e de Hopf (SEYDEL, 1994) são reconhecidas como as principais responsáveis pela instabilidade de tensão de sistemas de potência. Quando a matriz Jacobiana apresenta um autovalor simples igual a zero tem-se a bifurcação sela-nó. Esta é a mais comum em sistemas de potência correspondendo ao desaparecimento de um equilíbrio estável do sistema. Quando a matriz Jacobiana tem um simples par de autovalores complexos que atravessam o eixo imaginário do plano complexo, tem-se a bifurcação de Hopf. Neste caso, o comportamento dinâmico do sistema muda, passa do equilíbrio a uma oscilação.

\subsubsection{Bifurcações Sela-Nó e o Colapso de Tensão}

\subsubsection{Bifurcações Sela-Nó das Soluções de uma Equação Quadrática}

Bifurcação sela-nó é um fenômeno não-linear e não pode ocorrer em um modelo linear. Tal bifurcação está relacionada a um modelo não-linear como uma equação quadrática.

Seja uma equação quadrática com duas raízes reais (soluções de equilíbrio). Estas raízes deslocam-se gradualmente à medida que os coeficientes (parâmetros) desta equação quadrática sofrem variações também gradativas. A bifurcação ocorrerá quando raízes reais duplas forem a solução da equação. Este ponto solução estabelece uma fronteira entre a existência de duas raízes reais distintas e a inexistência de raízes reais. 
Existem, no modelo de um sistema elétrico de potência, variáveis que se relacionam através de equações quadráticas. Estas relações tornam o modelo nãolinear. Com isso, o sistema está sujeito à ocorrência de bifurcações selas-nós.

Seja o sistema mostrado na Figura 4:

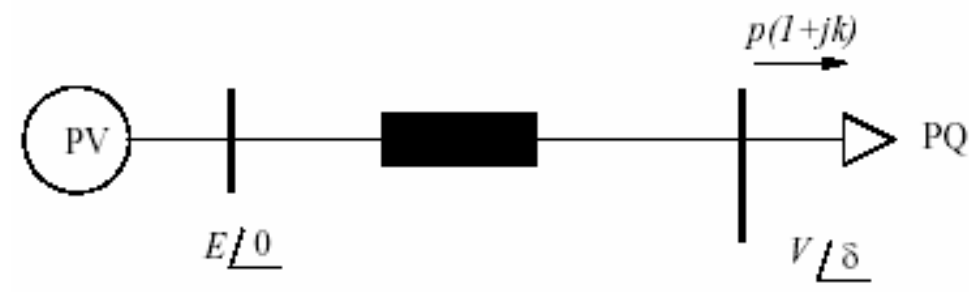

Figura 4 - Consumo de potência ativa

Foi escolhida a potência ativa $p$ como parâmetro para o sistema. $\mathrm{O}$ vetor de estados do sistema, $\mathbf{z}=(V, \delta)$, especifica o fasor de tensão para a carga. A variação da magnitude da tensão $V$ com o carregamento $p$ é mostrado na Figura 5:

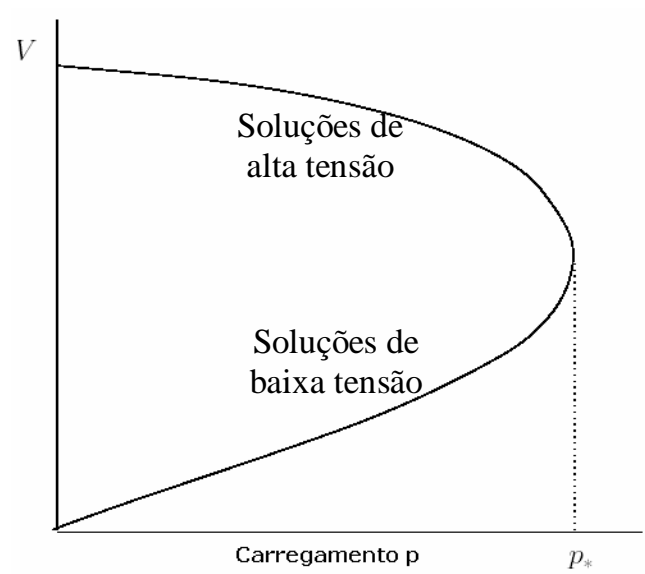

Figura 5 - Variação da magnitude de tensão com o carregamento

Para um baixo valor de carregamento existem duas soluções de equilíbrio, uma solução de alta tensão e outra de baixa tensão, que não apresenta nenhuma relação com o problema de baixa tensão descrito no item 3.3. Agregada à solução de alta tensão têm-se valores inferiores de corrente em comparação com a solução de baixa tensão. $\mathrm{Na}$ prática, as soluções de alta tensão são estáveis enquanto as de baixa tensão são instáveis. Com o acréscimo do carregamento, estas soluções se aproximam uma da outra até se tornarem uma única solução. Tem-se, portanto, o ponto de máximo carregamento (PMC) ou ponto de colapso de tensão. Acréscimos a 
partir deste ponto determinam a inexistência de soluções de equilíbrio. As soluções de equilíbrio desaparecem numa bifurcação sela-nó no ponto $p_{*}$.

Uma análise com relação às variáveis de estado que representam os ângulos de fase do sistema indica que a solução angular inferior corresponde à solução de alta tensão.

As extremidades das curvas $p V$ e $p \delta$ sinalizam o mesmo evento de equilíbrio instável e estável e, portanto, ocorrem para um mesmo carregamento $p_{*}$.

\subsubsection{Equilíbrios Estável e Instável}

É de grande utilidade visualizar o espaço de estados para várias condições de carregamento. As coordenadas para o espaço de estados são as variáveis $V$ e $\delta$.

A Figura 6 mostra ambos os equilíbrios para um carregamento moderado. As linhas indicam a dinâmica do sistema. Se um estado é perturbado levemente, em qualquer direção, a partir de um ponto de alta tensão, equilíbrio estável, as linhas mostram que o estado retornará a um ponto de equilíbrio estável. Por outro lado, quase todas as perturbações leves que ocorrerem a partir de um ponto de baixa tensão ocasionarão deslocamentos para pontos de equilíbrio instáveis.

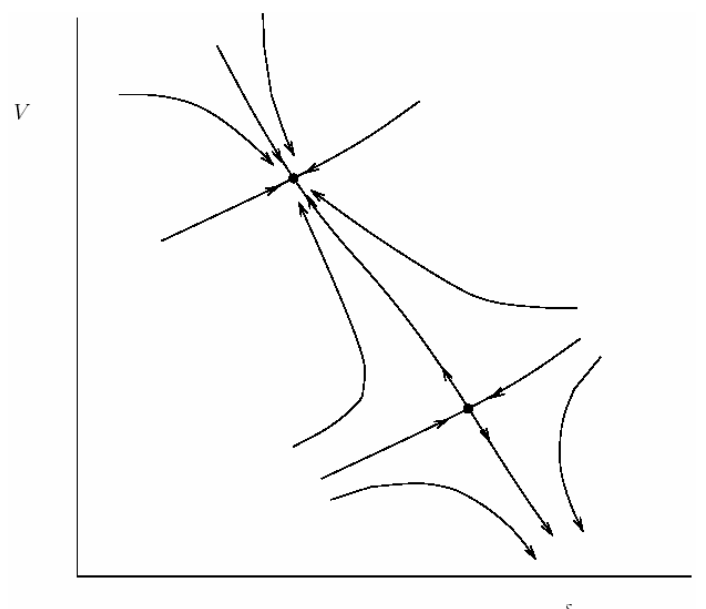

Figura 6 - Equilíbrios estável e instável para um carregamento moderado

Para o ponto de máximo carregamento mostrado na Figura 5, ponto de bifurcação $p_{*}$, as linhas mostram que este equilíbrio é instável. Além disso, tal instabilidade tende a mover o estado ao longo da seta em destaque na Figura 7. Este 
caminho implica em um declínio monotônico da magnitude da tensão e em um acréscimo do ângulo da tensão.

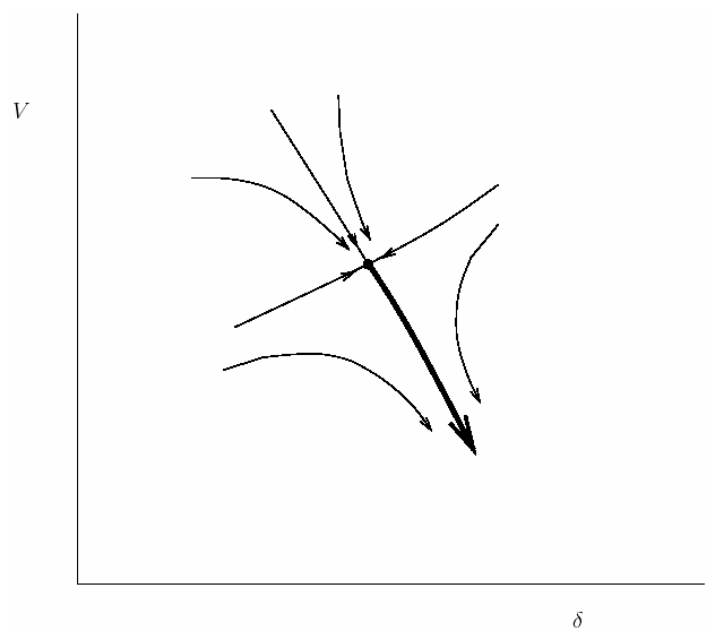

Figura 7 - Equilíbrio instável para um carregamento máximo

Em algumas situações de falta, o sistema de potência pode apresentar um carregamento maior que aquele do ponto de bifurcação. Neste caso, não existe um ponto de equilíbrio e a dinâmica do sistema está mostrada na Figura 8.

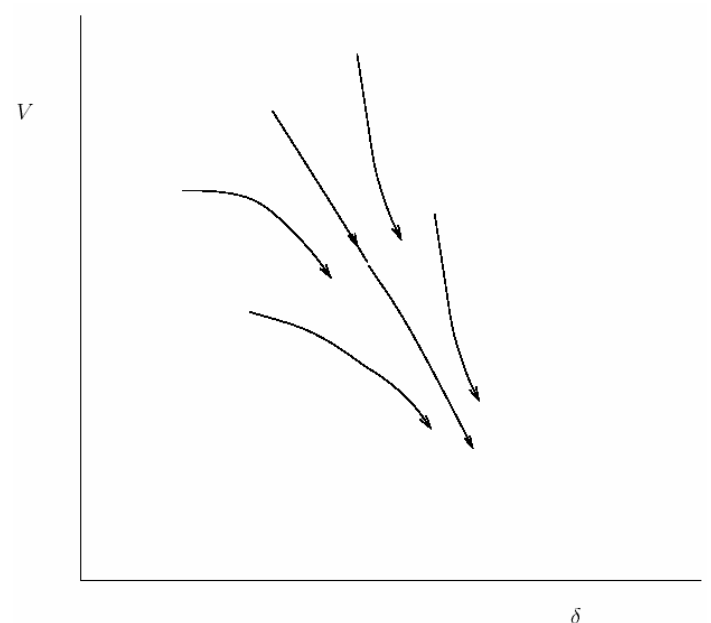

Figura 8 - Equilíbrio inexistente para um carregamento maior que o máximo

\subsubsection{Autovalores no Ponto de Bifurcação Sela-Nó}

Se a matriz Jacobiana de um sistema é estável assintoticamente, todos os autovalores têm parte real negativa. À medida que o sistema se aproxima do PMC, os autovalores da matriz Jacobiana aproximam-se do zero pelo lado esquerdo do plano 
complexo. A bifurcação ocorre quando o autovalor é zero. A matriz Jacobiana permite, portanto, a determinação da estabilidade de um sistema linearizado em relação ao seu equilíbrio. Logo, este equilíbrio deve existir. Se o PMC é ultrapassado, não existe equilíbrio nas proximidades e o uso da matriz Jacobiana perde seu significado.

Alguns Atributos de um Ponto de Bifurcação Sela-nó

Todas as indicações abaixo ocorrem em um ponto de bifurcação sela-nó e podem ser usadas para caracterizar ou detectar uma bifurcação sela-nó:

- dois pontos de equilíbrio unidos (um destes pontos deve ser instável);

- a sensibilidade em relação ao parâmetro de carregamento de uma variável de estado típica é infinita. Isto resulta em uma inclinação infinita na extremidade da curva como mostrado na Figura 5;

- a matriz Jacobiana do sistema tem um autovalor nulo;

- a matriz Jacobiana do sistema tem um valor singular nulo;

- o determinante da matriz Jacobiana é nulo;

- as dinâmicas do colapso, na bifurcação, são tais que o estado do sistema muda monotonicamente e a taxa de mudança é lenta inicialmente e rápida posteriormente.

\subsubsection{Diagramas de Bifurcação}

A extremidade de uma curva PV representará um ponto de bifurcação quando uma variável de estado estiver no eixo vertical e um parâmetro no eixo horizontal. Assim, este ponto corresponderá a uma bifurcação sela-nó, caracterizando a ocorrência de um colapso de tensão. Entretanto, nem todo parâmetro escolhido deve ser, necessariamente, um valor de potência de carga. Nesta situação, à medida que o parâmetro é variado, a potência de carga (no eixo horizontal) e a tensão na barra (no eixo vertical) também sofrem variações produzindo uma curva semelhante, mas que 
não representa um diagrama de bifurcação. Uma bifurcação sela-nó poderá ocorrer em qualquer ponto da curva, sem nenhuma relação à sua extremidade, que continuará sendo um ponto de máxima transferência de potência. Utilizando-se o "verdadeiro parâmetro", a curva PV gerada corresponderá a um diagrama de bifurcação cuja extremidade representará o ponto de bifurcação sela-nó.

\subsection{Modelos de Carga e Características PV da Rede}

A dependência das cargas em relação à tensão é um aspecto crítico na análise de estabilidade de tensão. Diferentes cargas são representadas por modelos que agregam comportamentos estáticos e dinâmicos para aproximar o efeito da sensibilidade destas em relação à tensão e à freqüência. Alguns modelos de cargas são apresentados a seguir, de acordo com ZAMBRONI DE SOUZA (1995).

Modelo Estático: um modelo que expressa as potências ativa e reativa, em qualquer instante de tempo, como funções da magnitude da tensão e da frequiência de uma barra naquele mesmo instante. Modelos estáticos de carga são usados para componentes estáticos como, por exemplo, cargas resistivas. Os modelos estáticos são divididos em:

- impedância constante: modelo em que a potência é diretamente proporcional ao quadrado da magnitude da tensão. Também pode ser denominado admitância constante;

- corrente constante: modelo em que a potência é diretamente proporcional à magnitude da tensão;

- potência constante: modelo em que a potência tem um valor constante frente a variações na magnitude da tensão;

- polinomial: modelo que apresenta uma equação polinomial relacionando potência e magnitude da tensão: 


$$
\begin{aligned}
& P=P_{0}\left[a_{1}\left(\frac{V}{V_{0}}\right)^{2}+a_{2}\left(\frac{V}{V_{0}}\right)+a_{3}\right] \\
& Q=Q_{0}\left[a_{4}\left(\frac{V}{V_{0}}\right)^{2}+a_{5}\left(\frac{V}{V_{0}}\right)+a_{6}\right]
\end{aligned}
$$

onde

- $P$ e $P_{0}$ são os valores atual e inicial de potência ativa, respectivamente.

- $a_{i}$ para $i=1, \ldots, 6$ são fatores multiplicadores.

- $V$ e $V_{0}$ são os valores atual e inicial do nível de tensão.

- $Q$ e $Q_{0}$ são os valores atual e inicial de potência reativa.

- exponencial: modelo que apresenta uma equação exponencial relacionando potência e magnitude da tensão:

$$
\begin{aligned}
& P=P_{0}\left(\frac{V}{V_{0}}\right)^{n p} \\
& Q=Q_{0}\left(\frac{V}{V_{0}}\right)^{n q}
\end{aligned}
$$

- $n p$ e $n q$ são fatores exponenciais.

Modelo Dinâmico: um modelo que expressa as potências ativa e reativa, em qualquer instante de tempo, como funções da magnitude da tensão e da freqüência em instantes passados de tempo e, usualmente, incluindo o instante atual. Diferenças ou equações diferenciais podem ser usadas para representar tais modelos.

A análise conceitual baseada nas curvas PV's considera as características da carga como função da tensão. Por exemplo, para uma carga que pode ser modelada como do tipo potência constante, a representação é ainda mais simples: a característica é uma reta vertical no plano PV. 
Em contraste com a característica da carga, trabalha-se também, com as características PV da rede elétrica, que dizem respeito à sua capacidade de manter o necessário equilíbrio entre tensão e potência ativa e reativa supridas (SIMÕES COSTA, 2002). Um exemplo de característica PV é mostrado, a seguir, para um sistema sem perdas, com fator de potência unitário $\left(Z_{L}=R_{L}\right)$ e sem a ação de dispositivos de controle e proteção.

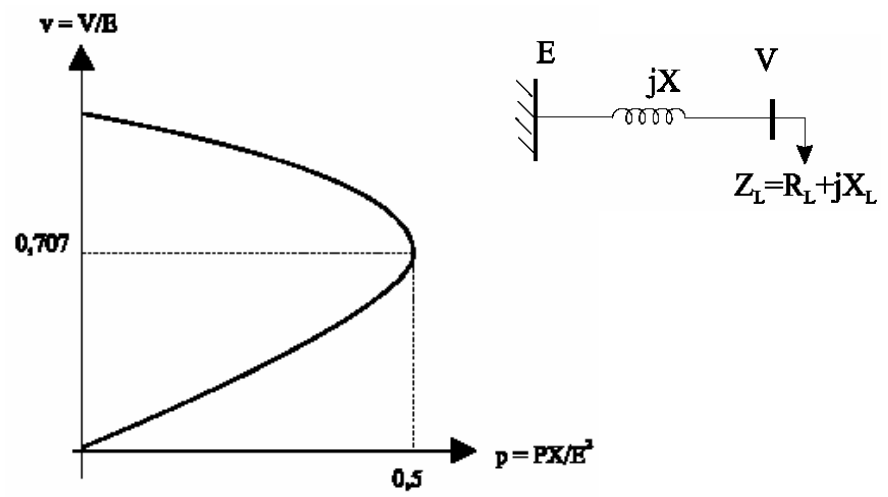

Figura 9 - Característica PV para um sistema sem perdas, com fator de potência unitário e sem a ação de dispositivos de controle e proteção

O desenvolvimento para fator de potência unitário pode ser generalizado para diferentes valores de fator de potência. Obtém-se, assim, a família de curvas PV's mostradas na Figura 10, onde o ângulo do fator de potência da carga é $\phi$.

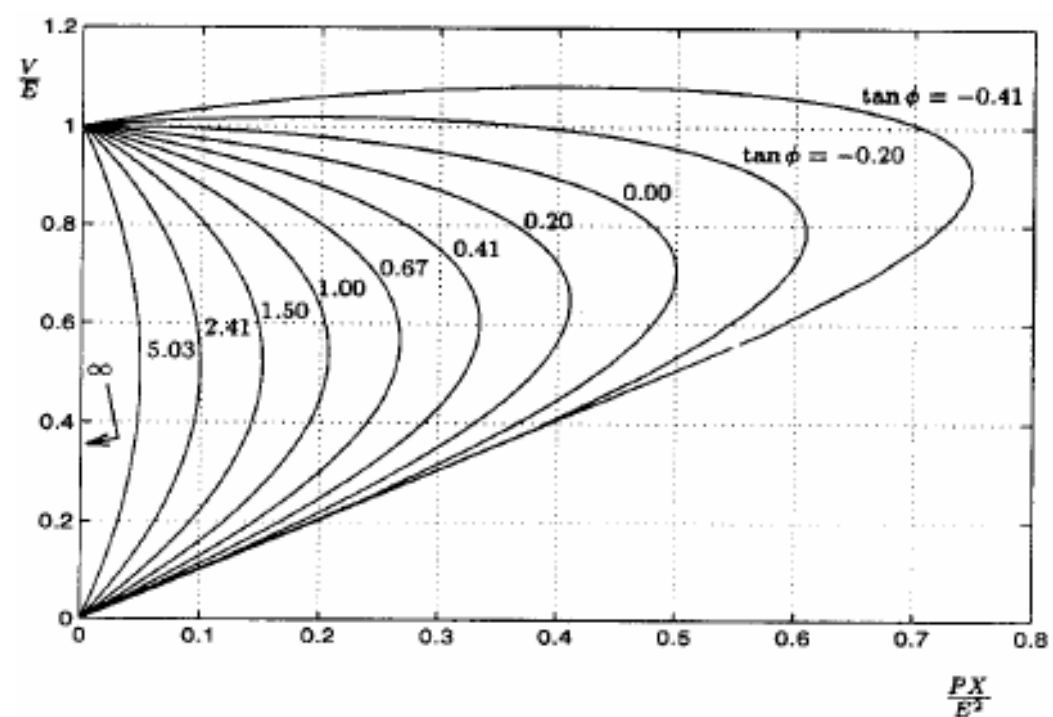

Figura 10 - Família de curvas PV's para diferentes valores de fator de potência 
Observa-se que:

- para uma dada carga abaixo do máximo carregamento possível, há duas soluções: uma delas para tensão mais alta e baixa corrente, e a outra para tensão mais baixa e alta corrente. A primeira corresponde às condições de operação normal com tensão mais próxima à tensão do gerador E. A operação permanente nas soluções do ramo inferior da curva é, em geral, inaceitável;

- à medida que a carga é compensada (o que corresponde a menores valores de $\operatorname{tg} \phi$ ) a potência máxima aumenta. Contudo, a tensão para a qual o máximo ocorre também aumenta. Esta situação indica um aumento de risco, já que a máxima capacidade de transferência de potência pode ser atingida para tensões próximas a valores normais de operação. Observa-se, também, que para um alto grau de compensação e uma potência ativa próxima do máximo, as duas soluções de tensão aproximam-se uma da outra. Torna-se mais difícil dizer, sem uma análise detalhada, qual das soluções é considerada "normal";

- para cargas sobre-compensadas $(\operatorname{tg} \phi<0)$, há uma parte do ramo superior da curva PV ao longo da qual a tensão aumenta com a potência da carga. A explicação é que, sob fator de potência negativo, quanto mais potência ativa é consumida, mais potência reativa é produzida pela carga. Em cargas baixas, a queda de tensão devido ao aumento de potência ativa é compensada pelo aumento de tensão devido ao aumento da potência reativa. Este efeito é mais pronunciado quanto mais negativo for $\operatorname{tg} \phi$.

Os pontos de operação possíveis no plano PV correspondem às interseções entre as características da rede e as características das cargas, como ilustrado na Figura 11. As características da rede se referem às características em regime permanente de geradores e sistemas de transmissão, enquanto que as cargas podem apresentar comportamentos distintos ao longo do tempo com respeito a variações de tensão. 


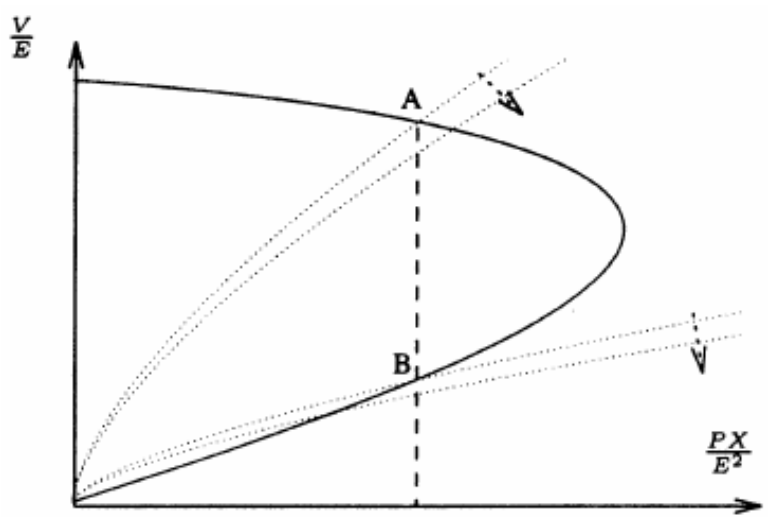

Figura 11 - Pontos de operação possíveis no plano PV

Um aspecto que deve ser ressaltado é que, dependendo do modelo estático utilizado para representar a carga agregada do sistema, a localização do ponto de sela-nó muda significativamente. No caso de um modelo de carga do tipo potência constante, a bifurcação sela-nó indicará o ponto de máxima transferência de potência ou de máximo carregamento, correspondendo à extremidade da curva. Em qualquer outro tipo de modelo de carga, o limite de carregamento não corresponderá ao limite de estabilidade (CAÑIZARES, 2001). Para [Pa192] apud ALVES (2000), cargas que exibem características de impedância ou corrente constantes não causam a instabilidade de tensão.

Algumas das aplicações para as curvas PV's são:

- levantar o perfil de tensão do sistema em diversas condições de carregamento;

- investigar a sensibilidade do estado do sistema com relação à variação do parâmetro;

- encontrar o PMC numa direção particular;

- encontrar pontos de múltiplas soluções. 


\section{CAPÍTULO 4}

\section{ÍNDICES DE ESTABILIDADE DE TENSÃO}

A obtenção de índices para prever a proximidade de um eventual colapso de tensão é de grande interesse entre pesquisadores e equipes técnicas na operação de sistemas de potência. Tais índices poderiam ser usados on-line ou off-line para auxiliar os operadores a determinar o grau de segurança de um sistema em relação a um possível colapso de tensão.

O objetivo destes índices é definir uma magnitude escalar que possa ser monitorada à medida que os parâmetros do sistema sofram variações. Estes índices deveriam apresentar um modelo previsível, sem sofrerem variações abruptas, para que previsões aceitáveis possam ser feitas. E além do mais, apresentar um baixo esforço computacional para um monitoramento on-line do sistema.

\subsection{Fatores de Sensibilidade}

Fatores de sensibilidade são índices bem conhecidos e usados em várias companhias de energia do mundo para detectar problemas de estabilidade de tensão (CAÑIZARES, 2001). Estes índices foram, primeiramente, utilizados para prever problemas no controle de tensão e podem ser definidos como:

$$
F S T_{i}=\max _{i}\left\{\frac{d V_{i}}{d Q_{i}}\right\},
$$

onde FST significa Fator de Sensibilidade de Tensão. Quando o gerador $i$ aproximase da porção inferior de sua curva $\mathrm{QV}$, o valor de $F S T$ torna-se grande e, ao trocar de sinal, indica uma instabilidade na condição de controle de tensão.

Baseados neste conceito, diversos índices têm sido propostos. A seguir, o índice FS, Fator de Sensibilidade, pode ser definido para um sistema, cuja representação matemática é feita através da equação $\mathbf{F}(\mathbf{z}, \lambda)$. 


$$
F S=\left\|\frac{d \mathbf{z}}{d \lambda}\right\|,
$$

onde um grande valor para $F S$ significa insegurança para o sistema em relação à possibilidade de ocorrer um colapso de tensão. Quando o sistema está próximo de um valor máximo para o parâmetro $\lambda(\Delta \lambda \rightarrow 0)$ tem-se $d z_{i} / d \lambda \rightarrow \pm \infty$. Se somente as tensões do sistema forem monitoradas, um equivalente $F S T$ pode ser definido:

$$
F S T=\left\|\frac{d \mathbf{V}}{d \lambda}\right\|
$$

Os índices FST e FS requerem muito pouco esforço computacional e, em sistemas de pequeno porte, permitem algumas previsões da proximidade do PMC. Porém, para sistemas maiores, tais índices não são muito sensíveis a variações no sistema. Deste modo, as informações fornecidas com relação à possibilidade de ocorrência do colapso de tensão não são confiáveis. Entretanto, medidas corretivas podem ser desenvolvidas.

\subsection{Valores Singulares e Autovalores}

\subsubsection{Valores Singulares}

A decomposição em valores singulares é, tipicamente, usada na determinação do posto da matriz, que é igual ao número de valores singulares não nulos da matriz Jacobiana. Os valores singulares têm sido usados em sistemas de potência devido ao grande uso da decomposição ortonormal da matriz Jacobiana (ZAMBRONI DE SOUZA, 1995). A aplicação destes valores em análise de colapso de tensão concentra-se no monitoramento do menor valor singular, o qual se torna zero no ponto de colapso de tensão.

Em geral, a matriz Jacobiana contém derivadas primeiras do resíduo de potência reativa $\mathbf{Q}(\mathbf{z}, \lambda)$ em relação às magnitudes de tensão $\mathbf{V} \in \mathbf{z}$. Logo, linearizando as equações em estado permanente $\mathbf{F}(\mathbf{z}, \lambda)=0$, no ponto de equilíbrio $\left(z_{0}, \lambda_{0}\right)$, tem-se: 


$$
\begin{gathered}
\Delta \mathbf{F}(\mathbf{z}, \lambda)=\mathbf{J} \Delta \mathbf{z} \\
\Rightarrow\left[\begin{array}{c}
\Delta \mathbf{P}(\boldsymbol{\delta}, \mathbf{V}, \lambda) \\
\Delta \mathbf{Q}(\boldsymbol{\delta}, \mathbf{V}, \lambda)
\end{array}\right]=\left[\begin{array}{ll}
\frac{\partial \mathbf{P}}{\partial \boldsymbol{\delta}}\left(z_{0}, \lambda_{0}\right) & \frac{\partial \mathbf{P}}{\partial \mathbf{V}}\left(z_{0}, \lambda_{0}\right) \\
\frac{\partial \mathbf{Q}}{\partial \boldsymbol{\delta}}\left(z_{0}, \lambda_{0}\right) & \frac{\partial \mathbf{Q}}{\partial \mathbf{V}}\left(z_{0}, \lambda_{0}\right)
\end{array}\right]\left[\begin{array}{c}
\Delta \boldsymbol{\delta} \\
\Delta \mathbf{V}
\end{array}\right]=\left[\begin{array}{ll}
\mathbf{J}_{1} & \mathbf{J}_{2} \\
\mathbf{J}_{3} & \mathbf{J}_{4}
\end{array}\right]\left[\begin{array}{c}
\Delta \boldsymbol{\delta} \\
\Delta \mathbf{V}
\end{array}\right]
\end{gathered}
$$

Assumindo que $\Delta \mathbf{P}(\mathbf{z}, \lambda)=0$ no ponto de colapso de tensão, pode-se escrever:

$$
\Delta \mathbf{Q}(\boldsymbol{\delta}, \mathbf{V}, \lambda)=\left(\mathbf{J}_{4}-\mathbf{J}_{3} \mathbf{J}_{1}^{-1} \mathbf{J}_{2}\right) \Delta \mathbf{V}=\mathbf{J}_{Q V} \Delta \mathbf{V}
$$

De acordo com a fórmula de Schur (GANTMACHER, 1977):

$$
\operatorname{det} \mathbf{J}=\operatorname{det} \mathbf{J}_{1} \operatorname{det} \mathbf{J}_{Q V},
$$

$\mathbf{J}$ é singular quando $\mathbf{J}_{Q V}$ ou $\mathbf{J}_{1}$ for singular. Caso não existam problemas de estabilidade de ângulo ( $\operatorname{det} \mathbf{J}_{1} \neq 0$ ), a matriz $\mathbf{J}$ se tornará singular se, e somente se, a matriz $\mathbf{J}_{Q V}$ se tornar singular (HILL, 1990). Portanto, os valores singulares desta matriz reduzida podem ser usados para determinar a proximidade do colapso de tensão.

\subsubsection{Autovalores}

Os autovalores, assim como os valores singulares, também têm sido utilizados na determinação da proximidade do ponto de colapso de tensão. Para a matriz $\mathbf{J}_{Q V}$, definida anteriormente, espera-se obter autovalores e autovetores reais similares aos valores correspondentes aos vetores e valores singulares. Assim, os autovetores associados aos autovalores próximos de zero possuem a mesma interpretação dos vetores singulares próximos do $\mathrm{PMC}$, isto é, os maiores valores de um autovetor à direita correspondem às barras com tensões mais sensíveis do sistema; e os maiores valores de um autovetor à esquerda indicam as direções mais sensíveis a variações na injeção de potência (KUNDUR et al., 1992).

Comparando-se os índices baseados nos valores singulares e autovalores em relação aos baseados nos fatores de sensibilidade, informações similares podem ser obtidas. Porém os primeiros apresentam um alto custo computacional.

Um fato que deve ser observado em relação aos valores singulares e autovalores é que o significado destes sofrem alterações expressivas de acordo com o 
modelo adotado para o sistema. Por exemplo, valores obtidos a partir de um modelo de fluxo de potência não fornecem informações válidas quanto à dinâmica do problema. Sendo assim, um estudo que utilize valores singulares e autovalores como índice de proximidade do carregamento máximo, para ser válido, deve conter elementos dinâmicos.

\subsection{Margem de Carregamento}

Para um ponto de operação particular, a quantidade adicional de carga que causaria um colapso de tensão, em um modelo específico de acréscimo da mesma, é denominada margem de carregamento para o colapso de tensão. A margem de carregamento é o mais básico e amplo índice de colapso de tensão aceito.

Se a carga de um sistema é escolhida para ser o parâmetro de carregamento, uma curva PV pode ser traçada para o mesmo; neste caso, a margem de carregamento para o colapso de tensão é a variação de carga entre o ponto de operação e a extremidade da curva.

Existem diversas maneiras para se definir a margem de carregamento. Uma alteração do valor da carga pode ser medida ou pela soma das variações absolutas da potência aparente da carga, ou pela raiz quadrada da soma dos quadrados das variações da potência aparente da mesma carga.

As vantagens da margem de carregamento, como índice para o colapso de tensão, são:

- a margem de carregamento é direcionada, de fácil aceitação e entendimento;

- a margem de carregamento não é baseada em um modelo particular para o sistema; requer, somente, um modelo estático do sistema de potência;

- a margem de carregamento é um índice preciso que considera a não linearidade do sistema e limites, como os de potência reativa, atingidos com o acréscimo da carga; 
- uma vez que a margem de carregamento é calculada, é rápido e fácil determinar sua sensibilidade em relação a quaisquer parâmetros ou controles do sistema de potência.

As desvantagens da margem de carregamento, como índice para o colapso de tensão, são:

- a margem de carregamento requer cálculos em pontos além do ponto de operação atual, por isso, é computacionalmente mais dispendiosa que índices que utilizam somente informações do ponto de operação. Os custos computacionais são as maiores desvantagens da margem de carregamento;

- a margem de carregamento requer a consideração de uma direção para o acréscimo de carga. Algumas vezes, esta informação não é avaliada corretamente.

Existem duas alternativas para amenizar a dependência da margem de carregamento quanto ao modelo de carregamento adotado. Uma delas é calcular a sensibilidade da margem de carregamento para o modelo de aumento de carga assumido ou recalcular a margem de carregamento para diferentes modelos. A outra consiste na realização de cálculos adicionais para encontrar um modelo de carregamento que forneça um valor mínimo para a margem de carregamento.

A margem de carregamento pode ser calculada fazendo-se pequenos incrementos na carga e recalculando os fluxos de potência em cada novo valor pósincremento até ser atingido a extremidade da curva. Na prática, elaborações desta idéia são utilizadas, como os métodos diretos e da continuação.

\subsubsection{Métodos Diretos}

Métodos diretos, também conhecidos como Métodos do PMC, foram desenvolvidos para calcular pontos de bifurcação singular de sistemas não lineares sem a determinação dos demais pontos existentes entre o caso base e o PMC 
(CAÑIZARES \& ALVARADO, 1993). O método consiste na solução das equações abaixo, para $\mathbf{z}, \lambda$ e $\mathbf{w}$ (autovetor à direita) obtidos do $\operatorname{PMC}\left(z_{*}, \lambda_{*}\right)$ :

$$
\begin{gathered}
\mathbf{F}(\mathbf{z}, \lambda)=0 \\
D_{z} \mathbf{F}(\mathbf{z}, \lambda)^{T} \mathbf{w}=0 \\
\|\mathbf{w}\|_{\infty}=1
\end{gathered}
$$

Este método permite a determinação direta da margem de carregamento para o colapso $\Delta \lambda=\lambda_{*}-\lambda$, em qualquer ponto de operação definido por $\lambda$, sem a necessidade de calcular os demais pontos existentes entre o caso base e a extremidade da curva.

Uma desvantagem deste método é o alto custo computacional: a dimensão total do sistema de equações anterior é de $2 n+1$, o dobro da matriz Jacobiana convencional, o que impõe maiores requisitos de memória e de tempo de CPU.

A utilização da norma infinita na terceira equação do sistema provê o modo mais confiável para encontrar o ponto de colapso de tensão. E a utilização do autovetor à direita, w, se deve à obtenção de melhores resultados quando da utilização deste em comparação com o autovetor à esquerda.

Equações similares podem ser obtidas para determinar o PMC como um problema de otimização (IRISARRI et al., 1997). Assim, o problema de colapso pode ser escrito como:

$$
\begin{array}{ll}
\max & \lambda \\
\text { sujeito } & a \mathbf{F}(\mathbf{z}, \lambda)=0
\end{array}
$$

Isto pode ser resolvido usando a função Lagrangiana:

$$
L(\mathbf{z}, \lambda, \boldsymbol{\omega})=\lambda+\omega^{T} * \mathbf{F}(\mathbf{z}, \lambda)
$$

onde $\omega$ corresponde ao vetor dos multiplicadores de Lagrange. Portanto, a satisfação das condições necessárias de primeira ordem é obtida da solução do Sistema 4.10:

$$
\begin{aligned}
& D_{\omega} L=\mathbf{F}(\mathbf{z}, \lambda)=0 \\
& D_{z} L=D_{z} \mathbf{F}(\mathbf{z}, \lambda)^{T} \boldsymbol{\omega}=0 \\
& \frac{\partial L}{\partial \lambda}=\omega^{T} \frac{\partial \mathbf{F}}{\partial \lambda}(\mathbf{z}, \lambda)+1=0
\end{aligned}
$$


Várias técnicas de otimização podem ser empregadas para determinar o PMC ao expressar o problema de colapso como um problema de otimização. Devido à capacidade de manipulação de variáveis canalizadas, um método, especialmente atraente, de otimização, é o de pontos interiores (IRISARRI et al., 1997).

\subsubsection{Método da Continuação}

Os métodos da continuação podem ser implementados com qualquer conjunto de equações de equilíbrio (ou de regime permanente) de um sistema de potência. Em análises de estabilidade de tensão, estes métodos têm sido empregados para o cálculo da trajetória de soluções.

A idéia básica consiste na obtenção de sucessivas soluções do fluxo de carga, com o objetivo de traçar os perfis de tensão a partir de um caso base até o PMC (AJJARAPU \& CHRISTY, 1992). O processo iterativo deve ser inicializado cuidadosamente para assegurar que uma solução apropriada seja obtida. Uma inicialização inadequada poderá resultar em não convergência ou convergência para soluções não desejadas. Portanto, uma das características necessárias para o método seria a de possuir uma região de convergência tão ampla quanto possível, evitando-se assim, a possibilidade de erros de convergência.

Neste método são superadas as dificuldades de obtenção de sucessivas soluções do fluxo de carga, o que possibilita o traçado completo do perfil de tensão a partir da variação do valor do parâmetro $\lambda$ (parâmetro associado à carga do sistema) automaticamente, sem preocupação com as singularidades das equações do sistema de potência.

A estratégia do método é mostrada na Figura 12, onde o ponto de equilíbrio $\left(z_{1}, \lambda_{1}\right)$ é usado para calcular a direção do vetor $\Delta z_{1}$ e a variação $\Delta \lambda_{1}$ do parâmetro do sistema. 


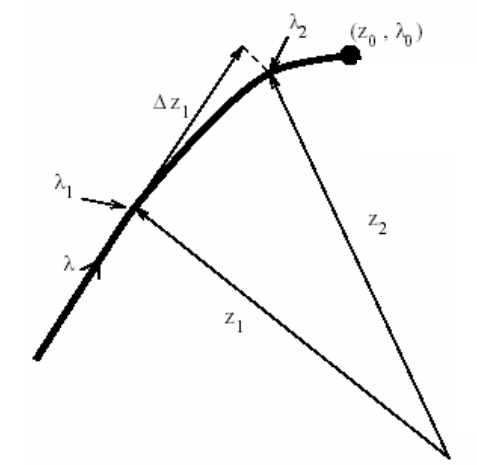

Figura 12 - Esquema preditor-corretor

Esta primeira etapa é conhecida como passo preditor. Com o resultado desta, tem-se uma estimativa para o próximo ponto $\left(z_{1}+\Delta z_{1}, \lambda_{1}+\Delta \lambda_{1}\right)$, que é usado depois numa segunda etapa, o passo corretor, para calcular um novo ponto de equilíbrio $\left(z_{2}, \lambda_{2}\right)$. Visto que a matriz Jacobiana é singular no ponto de bifurcação, uma parametrização se torna necessária nos passos preditor e/ou corretor, dependendo da técnica utilizada, para garantir um bom comportamento numérico dos resultados. A seguir, será apresentada uma descrição detalhada do assunto em questão baseada em CAÑIZARES (2001).

\subsubsection{Passo Preditor e Parametrização}

Uma forma de cálculo da direção do vetor $\Delta z_{1}$ no ponto de equilíbrio $\left(z_{1}, \lambda_{1}\right)$ é feita por meio do vetor tangente naquele ponto. Visto que $\mathbf{F}\left(z_{1}, \lambda_{1}\right)=0$, então:

$$
\begin{aligned}
& \left.D_{z} \mathbf{F}\left(z_{1}, \lambda_{1}\right) \frac{d \mathbf{z}}{d \lambda}\right|_{1}+\left.\frac{\partial \mathbf{F}}{\partial \lambda}\right|_{1}=0 \\
& \left.\left.\Rightarrow D_{z} \mathbf{F}\right|_{1} \frac{d \mathbf{z}}{d \lambda}\right|_{1}=-\left.\frac{\partial \mathbf{F}}{\partial \lambda}\right|_{1}
\end{aligned}
$$

Logo, a direção do vetor e o tamanho do passo a ser dado vêm da normalização do vetor tangente, ou seja: 


$$
\begin{gathered}
\Delta \lambda_{1}=\frac{k}{\left\|\left.\frac{d \mathbf{z}}{d \lambda}\right|_{1}\right\|} \\
\Delta z_{1}=\left.\Delta \lambda_{1} \frac{d \mathbf{z}}{d \lambda}\right|_{1},
\end{gathered}
$$

onde $k$ é uma constante escalar positiva que controla o tamanho do passo preditor. A normalização, na Equação 4.12, resulta na redução do tamanho do passo à medida que o sistema se aproxima do PMC, já que a magnitude do vetor tangente aumenta na proximidade deste ponto. Se o passo for muito grande, o ponto estimado $\left(z_{1}+\Delta z_{1}, \lambda_{1}+\Delta \lambda_{1}\right)$ apresentará problemas de convergência durante sua correção, enquanto que, para um passo muito pequeno, serão necessárias muitas iterações para atingir o ponto de bifurcação. Bons resultados foram encontrados em CAÑIZARES \& ALVARADO (1993) para $k=1$, considerando-se sistemas de diferentes portes. Uma técnica para determinar um tamanho adequado para o passo, baseada no vetor tangente, é proposta em ZAMBRONI DE SOUZA et al. (1997).

Uma dificuldade do método, entretanto, é quando o ponto de equilíbrio se encontra nas proximidades do ponto de bifurcação, onde a matriz Jacobiana se torna mal condicionada. Para evitar este problema, técnicas de parametrização podem ser usadas, como em SEYDEL (1994). Uma delas é a parametrização local, que consiste em alternar o parâmetro $\lambda$ com uma variável do sistema $z_{i} \in \mathbf{z}$ que possui o maior valor normalizado do vetor tangente. Assim, $\lambda$ se torna uma nova variável para o sistema e $z_{i}$ o novo parâmetro $p$ :

$$
p=\max _{i}\left\{\left|\frac{\Delta z_{i}}{z_{i}}\right|,\left|\frac{\Delta \lambda}{\lambda}\right|\right\}
$$

Outro tipo de preditor com parametrização é o do arco tangente. Nesta técnica, o parâmetro e as variáveis do sistema, no ponto de equilíbrio, podem ser representados como uma função do arco tangente $s$ do sistema. Isto é, para $\mathbf{F}\left(z_{1}(s), \lambda_{1}(s)\right)=0$,

$$
\left.\left.D_{z} \mathbf{F}\right|_{1} \frac{d \mathbf{z}}{d s}\right|_{1}+\left.\left.\frac{\partial \mathbf{F}}{\partial \lambda}\right|_{1} \frac{d \lambda}{d s}\right|_{1}=0
$$


onde o arco tangente $s$ deve satisfazer a condição:

$$
\left.\left.\frac{d \mathbf{z}}{d s}\right|_{1} ^{T} \frac{d \mathbf{z}}{d s}\right|_{1}+\left.\frac{d \lambda}{d s}\right|_{1} ^{2}=1
$$

Aproximando $\Delta \mathbf{z} \approx d \mathbf{z}, \Delta \lambda \approx d \lambda$ e $k=\Delta s \approx d s$, a Equação 4.14 pode ser escrita como:

$$
\begin{aligned}
& \left.D_{z} \mathbf{F}\right|_{1} \Delta z_{1}+\left.\frac{\partial \mathbf{F}}{\partial \lambda}\right|_{1} \Delta \lambda_{1}=0, \\
& \Delta z_{1}^{T} \Delta z_{1}+\Delta \lambda_{1}^{2}=k,
\end{aligned}
$$

onde $k$ é uma constante escalar positiva que define o tamanho do arco e, conseqüentemente, o tamanho do passo preditor.

Outro método preditor mais simples é o da secante, que não requer parametrização (CHIANG et al., 1995) e consiste na aproximação do vetor tangente $d z / d \lambda$ utilizando dois ou mais pontos determinados previamente. Desta forma, dados dois pontos $\left(z_{1 a}, \lambda_{1 a}\right)$ e $\left(z_{1 b}, \lambda_{1 b}\right)$, sendo $\lambda_{1 a}>\lambda_{1 b}$, tem-se:

$$
\left.\frac{d \mathbf{z}}{d \lambda}\right|_{1} \approx z_{1 b}-z_{1 a}
$$

Nota-se que, dentro de uma tolerância numérica razoável, a proximidade destes dois pontos é a melhor aproximação do vetor tangente; porém, um número maior de pontos deverá ser calculado. Por outro lado, pontos muito afastados um do outro geram aproximações inadequadas de $d \mathbf{z} / d \lambda$, causando dificuldades de convergência durante o passo corretor.

Dependendo da curvatura, o método secante apresenta vantagens e desvantagens em relação aos métodos preditores anteriores. Uma associação é proposta em IBA et al. (1991) e AJJARAPU \& CHRISTY (1992), onde se utiliza o preditor secante quando a inclinação do vetor tangente ainda é pequena. Na presença de curvaturas maiores, empregam-se os preditores arco tangente ou vetor tangente. A Figura 13 mostra o procedimento do preditor secante; e a Figura 14 descreve os problemas associados a esta técnica quando na presença de inclinações acentuadas. 


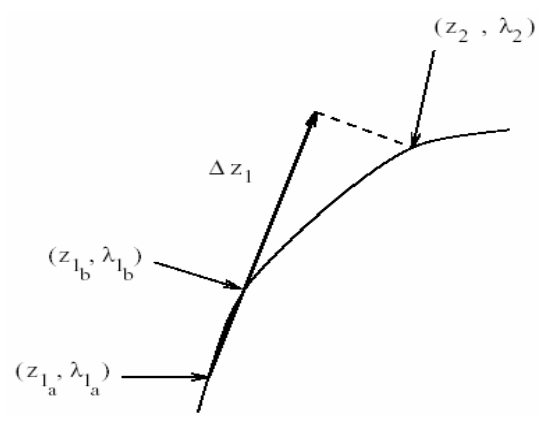

Figura 13 - Método preditor secante

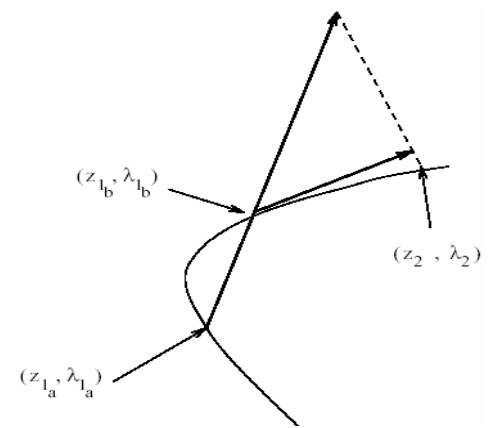

Figura 14 -Problema associado ao método preditor secante

\subsection{Alguns Detalhes sobre Parametrização}

De acordo com SEYDEL (1994), as curvas de solução da equação $\mathbf{f}(\mathbf{y}, \lambda)=\mathbf{f}(\mathbf{x})=0$ podem ser parametrizadas pelo parâmetro $\gamma$, o que significa que as soluções de $\mathbf{f}(\mathbf{y}, \lambda)=0$ dependem de $\gamma$ :

$$
\mathbf{y}=\mathbf{y}(\gamma), \lambda=\lambda(\gamma)
$$

Para um valor particular de $\gamma$, o sistema $\mathbf{f}(\mathbf{y}, \lambda)=0$ consiste de $n$ equações para $n+1$ incógnitas. Se a parametrização for estabelecida por uma equação escalar adicional, $p(\mathbf{y}, \lambda, \gamma)=0$, um sistema estendido pode ser formulado:

$$
\mathbf{F}(\mathbf{Y}, \gamma):=\left(\begin{array}{c}
\mathbf{f}(\mathbf{y}, \lambda) \\
p(\mathbf{y}, \lambda, \gamma)
\end{array}\right)=0
$$

o qual consiste de $n+1$ equações escalares para $n+1$ incógnitas, $\mathbf{Y}=(\mathbf{y}, \lambda)$. A Equação 4.19 inclui todos os tipos de parametrização. A opção de fazer $\lambda$ como 
parâmetro da continuação, significa escolher $\gamma=\lambda$ e $p(\mathbf{y}, \lambda)=\lambda-\lambda_{j+1}$, onde $j$ representa o valor atual.

\subsection{Parametrização Local}

É importante também, admitir alguns dos componentes $x_{i}(i=1, \ldots, n)$ como um parâmetro, incluindo $x_{n+1}=\lambda$. Esta parametrização local conduz à seguinte equação:

$$
p(\mathbf{x}, \eta):=x_{k}-\eta
$$

para $1 \leq k \leq n+1$ e um valor apropriado de $\eta$. O índice $k$ e o parâmetro $\eta=x_{k}$ são determinados localmente em cada etapa da continuação $\left(\mathbf{x}^{\mathrm{j}}, \lambda_{j}\right)$, a fim de manter sua flexibilidade. Assim, um índice $k$ para a parametrização local existe nas proximidades do ponto de solução única. $\operatorname{Com} \mathbf{Y}=(\mathbf{y}, \lambda)$, a Equação 4.19 pode ser escrita como:

$$
\mathbf{F}(\mathbf{Y}, \eta)=\left(\begin{array}{c}
\mathbf{f}(\mathbf{Y}) \\
x_{k}-\eta
\end{array}\right)=0
$$

Para encontrar um índice apropriado para $k$ e para o valor do parâmetro $\eta$, baseados no preditor tangente, tem-se a seguinte equação:

$$
\left|z_{k}\right|=\max \left\{\left|z_{1}\right|, \ldots,\left|z_{n}\right|,\left|z_{n+1}\right|\right\}
$$

Nesta escolha, determina-se a componente do vetor tangente $\mathbf{z}$ que é máxima. Para um algoritmo baseado no preditor secante, o índice $k$ é escolhido da seguinte forma:

$$
\begin{gathered}
\Delta_{r} x_{k}=\max \left\{\Delta_{r} x_{1}, \ldots, \Delta_{r} x_{n+1}\right\}, \\
\operatorname{com} \Delta_{r} x_{i}:=\frac{\left|x_{i}^{j}-x_{i}^{j-1}\right|}{\left|x_{i}^{j}\right|}
\end{gathered}
$$

Após um índice $k$ ser fixado, um valor para o parâmetro $\eta$ deve ser determinado. Este parâmetro depende do índice $k$, da posição da solução atual na curva (isto é, em $j$ ) e do tamanho do passo $\sigma$ desejado. 


$$
\eta=\eta(k, j, \sigma)
$$

\subsubsection{Passo Corretor}

A partir de uma estimativa inicial $\left(z_{1}+\Delta z_{1}, \lambda_{1}+\Delta \lambda_{1}\right)$ determinada no passo preditor, com ou sem parametrização, o ponto atual $\left(z_{2}, \lambda_{2}\right)$ deve ser calculado a partir das seguintes equações para $\mathbf{z}$ e $\lambda$ :

$$
\begin{aligned}
& \mathbf{F}(\mathbf{z}, \lambda)=0 \\
& \boldsymbol{\rho}(\mathbf{z}, \lambda)=0
\end{aligned}
$$

A equação $\mathbf{F}(\mathbf{z}, \lambda)=0$ corresponde às equações do fluxo de carga, $\left.D_{z} \mathbf{F}\right|_{*}=0$, no PMC, $\left(z_{*}, \lambda_{*}\right)$, sendo a matriz Jacobiana singular. A equação $\boldsymbol{\rho}(\mathbf{z}, \lambda)=0$ representa a condição que garante a não singularidade da matriz Jacobiana em todos os pontos do sistema:

$$
\mathbf{J}=\left[\begin{array}{ll}
D_{z} \mathbf{F} & \frac{\partial \mathbf{F}}{\partial \lambda} \\
D_{z} \boldsymbol{\rho} & \frac{\partial \boldsymbol{\rho}}{\partial \lambda}
\end{array}\right]_{(n+1, n+1)}
$$

Duas diferentes técnicas de correção têm sido usadas nos estudos de colapso de tensão em sistemas de potência. A primeira consiste em um vetor perpendicular à $\Delta z_{1}$, com origem no ponto $\left(z_{1}+\Delta z_{1}, \lambda_{1}+\Delta \lambda_{1}\right)$, que intersecta a curva PV em $\left(z_{2}, \lambda_{2}\right)$.

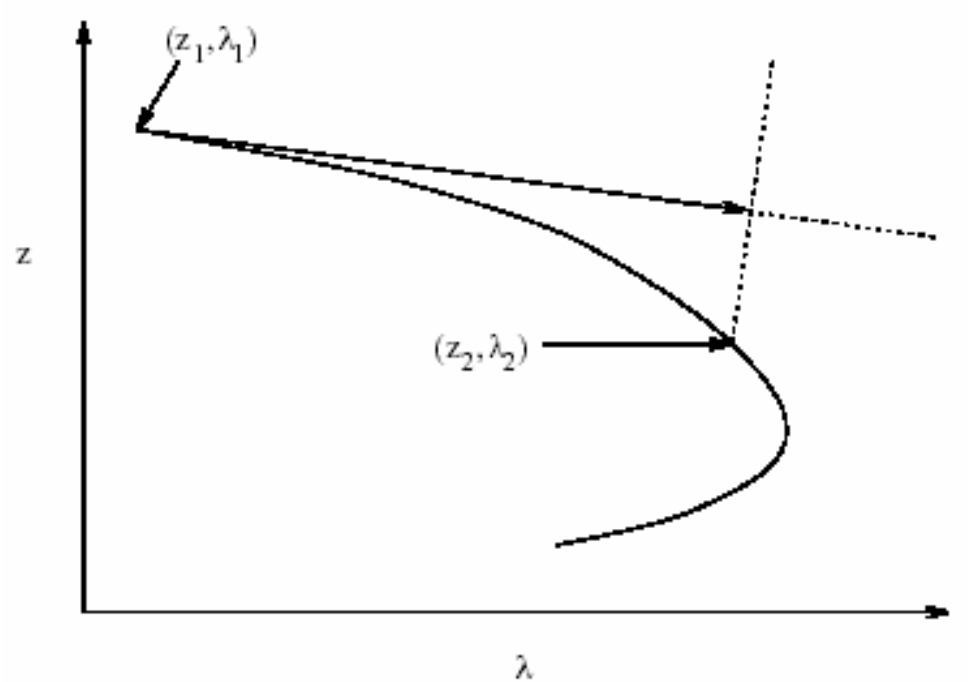

Figura 15 - Técnica de correção baseada em um vetor perpendicular 
Desta forma,

$$
\boldsymbol{\rho}\left(z_{2}, \lambda_{2}\right)=\Delta z_{1}^{T}\left(z_{2}-z_{1}-\Delta z_{1}\right)+\Delta \lambda_{1}\left(\lambda_{2}-\lambda_{1}-\Delta \lambda_{1}\right)
$$

Tal condição não requer qualquer tipo de parametrização para garantir a não singularidade da matriz Jacobiana (IBA et al., 1991).

A segunda técnica baseia-se em uma parametrização local em torno do PMC do sistema. Neste caso, um parâmetro local $p\left(\lambda\right.$ ou $\left.z_{i} \in \mathbf{z}\right)$ é usado como valor constante, ou seja:

$$
\boldsymbol{\rho}\left(z_{2}, \lambda_{2}\right)=p-p_{1}-\Delta p_{1}
$$

O parâmetro $p$ é escolhido conforme a Equação 4.22, garantindo a não singularidade da matriz Jacobiana. Tal técnica é ilustrada a seguir:

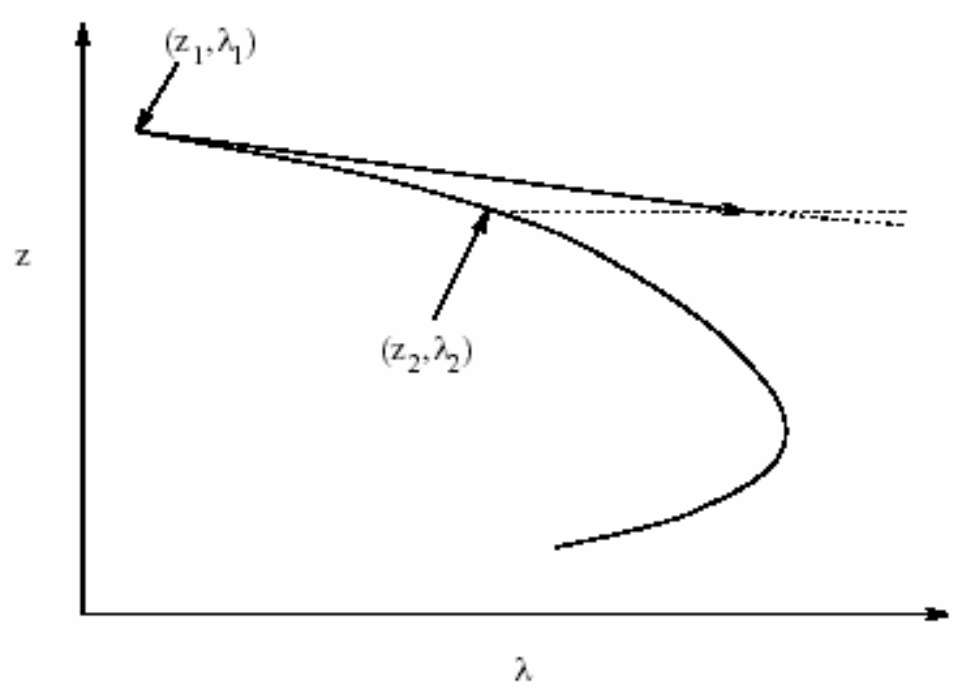

Figura 16 - Técnica de correção baseada em uma parametrização local

\subsection{Par de Soluções do Fluxo de Potência}

Para as situações onde são obtidas soluções reais para as equações do fluxo de potência observam-se dois valores diferentes (soluções de alta e baixa tensão) para a tensão em uma barra. Este par de soluções baseia-se no estudo das múltiplas soluções do fluxo de potência (IBA et al., 1990), onde a solução de baixa tensão é estimada utilizando-se a teoria do multiplicador ótimo (IWAMOTO \& TAMURA, 1981). 
A Equação $g_{0}+g_{1} \mu+g_{2} \mu^{2}+g_{3} \mu^{3}=0$ apresenta três raízes $\left(\mu_{1}<\mu_{2}<\mu_{3} \in\right.$ C, sendo C o conjunto dos números complexos). Na Equação 4.29, a atualização das magnitudes das tensões no cálculo da solução de alta tensão ou solução A é feita utilizando-se o multiplicador ótimo $\mu_{1}$. Entretanto, sua omissão não interfere no processo desde que este seja convergente. Caso contrário, um resultado, o mais próximo possível da solução, será determinado a partir da minimização da função $F u=\frac{1}{2} \sum_{i=1}^{2 n}\left(a_{i}+\mu b_{i}+\mu^{2} c_{i}\right)^{2}$, sem a convergência dos resíduos de potência. Este é o método Newton-Raphson com fator de passo otimizado ou, simplesmente, fluxo de potência de segunda ordem:

$$
\mathbf{V}_{A}^{(h+1)}=\mathbf{V}_{A}{ }^{(h)}+\mu_{1}^{(h)} \Delta \mathbf{V}_{A}^{(h)}
$$

O fluxograma da Figura 17 mostra a determinação da solução de baixa tensão ou solução B quando da convergência da solução A.

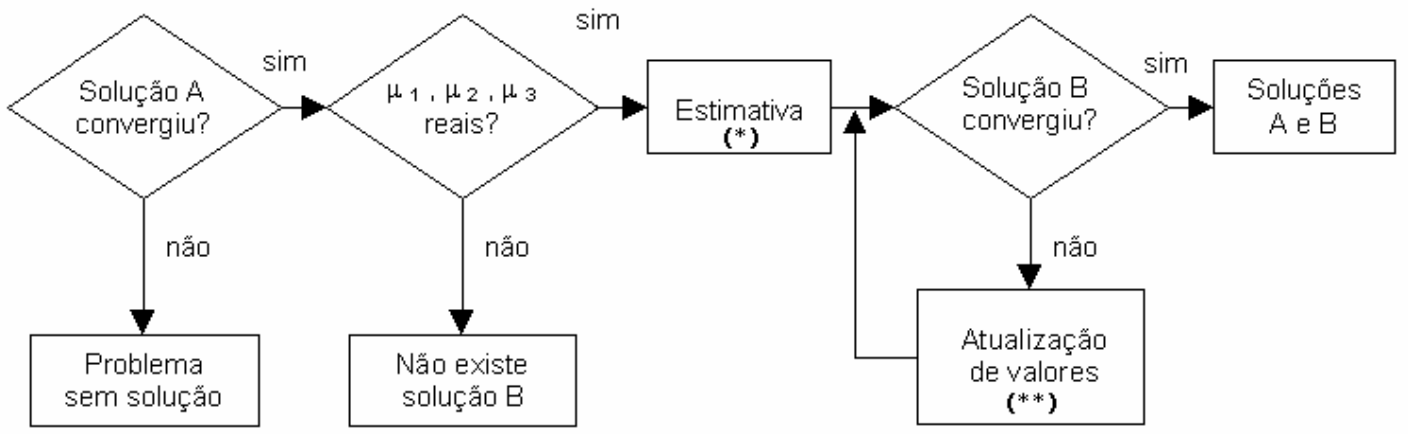

Figura 17 - Cálculo das soluções A e B
(*) $\mathbf{V}_{B}=\mathbf{V}_{A}+\mu_{3} \Delta \mathbf{V}_{A}$
(**) $\mathbf{V}_{B}=\mathbf{V}_{B}+\Delta \mathbf{V}_{B}$

\subsection{Funções Energia}

A análise a seguir oferece uma visão geométrica do colapso de tensão, como mostrado na Figura 18. Seja um ambiente repleto de montanhas e vales. Este ambiente pode sofrer alterações à medida que os parâmetros do sistema de potência, que correspondem àquele ambiente, também sejam alvos de variações. Ou seja, no lugar de um vale pode aparecer uma montanha. Os pontos de estabilidade estável se 
referem aos vales, enquanto que as montanhas irão representar limites que, quando ultrapassados, podem tornar o sistema instável (caracterizado por um declínio progressivo e incontrolável das tensões ou por oscilações de tensão com amplitudes crescentes). A altura da menor montanha pode ser medida por meio de uma função energia e depois usada como índice para monitorar o colapso de tensão.

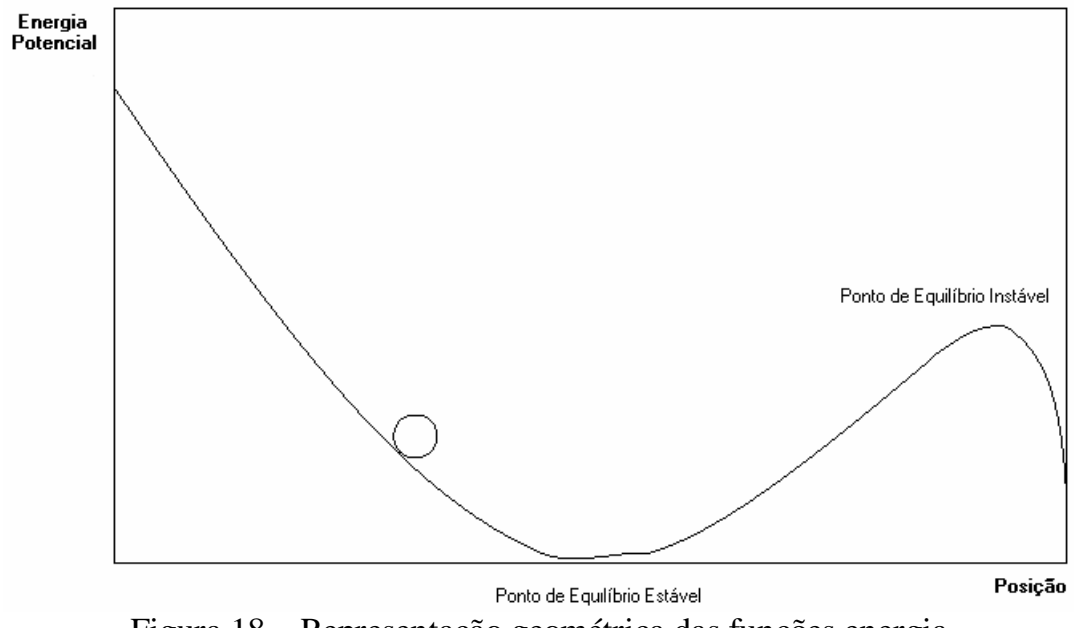

Figura 18 - Representação geométrica das funções energia

Diversos outros métodos foram desenvolvidos com o objetivo de determinar um índice que indique a proximidade do colapso de tensão. Em GUBINA \& STRMCNIK (1995), um índice é calculado a partir dos fasores de tensão. Em LEITE DA SILVA et al. (2000), o problema do colapso de tensão é revisto considerando a natureza estocástica da carga; uma metodologia probabilística é desenvolvida para fornecer índices de risco de colapso de tensão baseada numa combinação dos métodos do vetor tangente e da simulação Monte Carlo. Considerando-se a capacidade do sistema em gerar potência reativa, FLATABO et al. (1990) apresentam um método baseado em técnicas de sensibilidade linear.

Como foi ressaltado no item 3.5, é de suma importância conhecer as diferenças existentes entre o ponto de colapso de tensão (limite de estabilidade) e o ponto de máximo carregamento. Somente quando é adotado um modelo de carga do tipo potência constante, a bifurcação sela-nó indicará o ponto de máxima transferência de potência ou de máximo carregamento, correspondendo, deste modo, à extremidade da curva. 


\section{CAPÍTULO 5}

\section{MÉTODO DA CONTINUAÇÃO APLICADO AO PROBLEMA DE FLUXO DE POTÊNCIA}

\subsection{Introdução}

O método utilizado neste trabalho é o da continuação, para o qual existem variações, sendo que a diferença entre elas está na forma de tratar a nova variável lambda $(\lambda)$ e em como contornar a singularidade da matriz Jacobiana. Entre os diversos métodos da continuação descritos na literatura na área de sistemas de potência, o mais amplamente utilizado consiste de quatro elementos básicos: um passo preditor, um controle de passo, um passo corretor e um procedimento de parametrização.

Este método supera as dificuldades de obtenção de sucessivas soluções do fluxo de potência possibilitando assim, o traçado completo do perfil de tensão a partir da variação automática do parâmetro $\lambda$. Tal processo era realizado com a variação manual de $\lambda$ até a ocorrência da divergência. A proximidade do PMC, que torna iminente a singularidade da matriz Jacobiana, deixa de ser uma preocupação no método aqui utilizado.

A estratégia deste método foi apresentada na seção 4.3.2 e sua aplicação às equações de fluxo de potência será descrita a seguir, de forma detalhada, seguindo os moldes de AJJARAPU \& CHRISTY (1992).

\subsection{Reformulação das Equações do Fluxo de Potência}

Considerando-se que a carga varia igualmente por todo o sistema, mantendo o fator de potência constante, e que a variação da potência ativa das cargas não é assumida somente pela barra slack mas por todas as barras de geração disponíveis, o conjunto das equações do fluxo de potência, com a introdução do parâmetro $\lambda$, pode ser expressado como: 


$$
\mathbf{F}(\boldsymbol{\delta}, \mathbf{V}, \lambda)=0 \quad 0 \leq \lambda \leq \lambda_{\text {crítico }},
$$

onde $\boldsymbol{\delta}$ é o vetor dos ângulos de fase das tensões nas barras e $\mathbf{V}$ é o vetor das magnitudes das tensões nas barras. Reescrevendo a Equação 5.1, tem-se:

$$
\begin{gathered}
\lambda \mathbf{P}^{\text {esp }}+\mathbf{P}^{\text {esp }}-\mathbf{P}(\mathbf{V}, \boldsymbol{\delta})=0, \text { para barras } \mathrm{PV} \text { e } \mathrm{PQ}, \\
\lambda \mathbf{Q}^{\text {esp }}-\mathbf{Q}_{\mathrm{c}}^{\text {esp }}-\mathbf{Q}(\mathbf{V}, \boldsymbol{\delta})=0, \text { para barras } \mathrm{PQ},
\end{gathered}
$$

onde $\mathbf{P}^{\text {esp }}=\mathbf{P}_{\mathrm{g}}^{\text {esp }}-\mathbf{P}_{\mathbf{c}}^{\text {esp }}$ é a diferença entre as potências ativas, gerada e consumida nas barras de carga (PQ) e de geração (PV); e $\mathbf{Q}^{\text {esp }}=\frac{\mathbf{Q}_{\mathrm{g}}^{\text {esp }}}{\lambda}-\mathbf{Q}_{\mathbf{c}}^{\text {esp }}$, a diferença entre as potências reativas, gerada e consumida nas barras de carga (PQ). $\mathrm{O}$ valor de $\lambda$ igual a zero corresponde ao carregamento do caso base $\left(\boldsymbol{\delta}_{0}, \mathbf{V}_{0}, \lambda_{0}\right)$. Uma vez que todas as barras de geração disponíveis são responsáveis pela variação ativa das cargas tem-se: $\mathbf{P}_{\mathrm{g}}^{\text {esp }}=\lambda \mathbf{P}_{\mathrm{g}}^{\text {esp }}+\mathbf{P}_{\mathrm{g}}^{\text {esp }}$.

Segundo [Chia99] apud ALVES (2000), com relação à modelagem da carga (potência constante, corrente constante e impedância constante), adota-se a potência constante por resultar em pontos de máximo carregamento mais pessimistas, isto é, maiores possibilidades de ocorrência do colapso de tensão. Caso sejam necessárias, representações mais fiéis do modelo da carga podem ser utilizadas.

\subsection{Aplicação do Método da Continuação}

Após a introdução do parâmetro $\lambda$ nas equações do fluxo de potência, o algoritmo da continuação tem como ponto de partida a solução do caso base $\left(\lambda_{0}=0\right)$, calculado via fluxo de potência convencional. A etapa seguinte corresponde à predição da próxima solução, ou seja, a sua estimativa. A técnica de predição utilizada neste trabalho se refere ao método tangente. Neste método, será dado um passo na direção do vetor tangente ao ponto correspondente à solução atual. O cálculo deste vetor é mostrado na Equação 5.4:

$$
d \mathbf{F}(\boldsymbol{\delta}, \mathbf{V}, \lambda)=\mathbf{F}_{\boldsymbol{\delta}} d \boldsymbol{\delta}+\mathbf{F}_{\mathbf{V}} d \mathbf{V}+\mathbf{F}_{\lambda} d \lambda=0,
$$

que é representado matricialmente por: 


$$
\left[\begin{array}{lll}
\mathbf{F}_{\boldsymbol{\delta}} & \mathbf{F}_{\mathbf{V}} & \mathbf{F}_{\lambda}
\end{array}\right]\left[\begin{array}{c}
d \boldsymbol{\delta} \\
d \mathbf{V} \\
d \lambda
\end{array}\right]=0
$$

onde a matriz à esquerda é a matriz Jacobiana do fluxo de potência convencional aumentada em uma coluna $\left(\mathbf{F}_{\lambda}\right)$; e o vetor à direita, o vetor tangente.

Entretanto, surge um problema quando se introduz o parâmetro $\lambda$ : o sistema, antes representado por $n$ equações e $n$ incógnitas está, agora, representado por $n$ equações e $n+1$ incógnitas. Ou seja, de possível e determinado, o sistema passa a ser possível e indeterminado. Para superar este obstáculo é necessária mais uma equação. Desta forma, teríamos um sistema com $n+1$ equações e $n+1$ incógnitas. Há, porém, uma segunda alternativa: definir qualquer uma das $n+1$ variáveis como parâmetro. $\mathrm{O}$ valor deste parâmetro pode ser especificado e este, por fim, removido do sistema. Com isso, novamente ter-se-ia $n$ equações a $n$ incógnitas. O processo, indicado pela Equação 5.6, revela como se dá tal procedimento.

Considerando-se a solução predita, sua correção para um ponto convergido, utilizando o método de Newton apresentado em MONTICELLI (1983), é:

$$
\mathbf{g}(\mathbf{x})=-\mathbf{J}(\mathbf{x}) \Delta \mathbf{x} \therefore \Delta \mathbf{x}=-\mathbf{J}^{-1}(\mathbf{x}) \mathbf{g}(\mathbf{x}),
$$

onde:

$$
\mathbf{x}=\left[\begin{array}{c}
\boldsymbol{\delta} \\
\mathbf{V} \\
\lambda
\end{array}\right] \rightarrow n_{1}+n_{2}, \Delta \mathbf{x}=\left[\begin{array}{c}
\Delta \boldsymbol{\delta} \\
\Delta \mathbf{V} \\
\Delta \lambda
\end{array}\right] \rightarrow n_{1}+n_{1} \quad, \mathbf{g}(\mathbf{x})=\left[\begin{array}{c}
\Delta \mathbf{P} \\
\Delta \mathbf{Q} \\
0
\end{array}\right] \rightarrow n_{1}+n_{2}
$$

sendo $n_{l}$ e $n_{2}$ o número de barras PQ e PV respectivamente.

Em relação à inversa da matriz Jacobiana, sua modificação, quando da introdução de $\lambda$, equivale ao acréscimo de uma linha (equação) e uma coluna (variável). Esta nova linha deverá representar os coeficientes da nova equação, que terá como função fixar o valor de uma das variáveis. Sendo assim, todos os elementos serão nulos, exceto o k-ésimo, que será igual a 1 . Supondo-se que esta variável de valor fixo esteja na posição $k$, tem-se: 


$$
\mathbf{J}^{-1}(\mathbf{x})=\left[\begin{array}{ccccc}
j_{1,1} & \cdots & \cdots & \cdots & j_{1, q} \\
\vdots & \vdots & \vdots & \vdots & \vdots \\
0 & \cdots & 0 & \cdots & j_{k, q} \\
\vdots & \vdots & \vdots & \vdots & \vdots \\
j_{p, 1} & \cdots & \cdots & \cdots & j_{p, q}
\end{array}\right]_{(\mathrm{p}, \mathrm{q})=(\mathrm{n}+1, \mathrm{n}+1)}
$$

o que implica em:

$$
\left[\begin{array}{c}
\Delta x_{1} \\
\vdots \\
\Delta x_{k} \\
\vdots \\
\Delta x_{\mathrm{q}}
\end{array}\right]=-\left[\begin{array}{ccccc}
j_{1,1} & \cdots & \cdots & \cdots & j_{1, q} \\
\vdots & \vdots & \vdots & \vdots & \vdots \\
0 & \cdots & 0 & \cdots & 1 \\
\vdots & \vdots & \vdots & \vdots & \vdots \\
j_{p, 1} & \cdots & \cdots & \cdots & j_{p, q}
\end{array}\right]\left[\begin{array}{c}
g\left(x_{1}\right) \\
\vdots \\
g\left(x_{k}\right) \\
\vdots \\
0
\end{array}\right]
$$

Ao solucionar o sistema acima, somente para a $k$-ésima variável, tem-se:

$$
\Delta x_{k}=-\left[0 . g\left(x_{1}\right)+\cdots+0 . g\left(x_{k}\right)+\cdots+1.0\right]=0
$$

Tal variável é comumente conhecida como parâmetro de continuação, que é de fundamental importância no processo de correção da solução predita.

Existem diversos procedimentos para a escolha da variável a ser empregada como parâmetro de continuação. Matematicamente, a variável "contemplada" será aquela que apresentar a maior taxa de variação próxima a uma solução dada. Analisando-se em termos de sistemas de potência, $\lambda$ como parâmetro de continuação é a melhor escolha quando o processo tem início a partir de um caso base, especialmente se o caso base é caracterizado por um carregamento leve. Sob tais circunstâncias, os ângulos e magnitudes das tensões sofrem alterações mínimas. Por outro lado, quando a solução se aproxima do PMC, ângulos e magnitudes de tensões passam a experimentar mudanças significativas, o contrário do parâmetro de carregamento $\lambda$, que seria uma péssima escolha nesta situação.

Por esta razão, a escolha do parâmetro de continuação deve ser recalculada a cada passo do processo de acordo com a equação:

$$
x_{k}:\left|t_{k}\right|=\max \left\{\left|t_{1}\right|,\left|t_{2}\right|, \cdots\left|t_{\mathrm{m}}\right|\right\},
$$

onde t é o vetor tangente com dimensão igual a $m=2 n_{1}+n_{2}+1$ e $k$ corresponde ao máximo componente do vetor tangente (SEYDEL, 1994). Deste modo, nas equações 
da rede elétrica, ângulos de fase e magnitudes de tensão nas barras, assim como o próprio parâmetro de carregamento, podem se tornar parâmetros de continuação.

A parametrização local é aquela em que cada uma das variáveis de estado presentes no problema pode ser um parâmetro de continuação para diferentes soluções preditas. O equacionamento que representa a correção para uma solução convergida pode ser indicado por:

$$
\left[\begin{array}{c}
\mathbf{F}(\mathbf{x}) \\
x_{k}-\eta
\end{array}\right]=0
$$

onde $\eta$ é o valor predito para o k-ésimo elemento de $\mathbf{x}$.

Uma vez que o problema original do fluxo de potência sofreu modificações, a nova matriz Jacobiana ( $\mathbf{J}$ ) para o sistema é:

$$
\begin{gathered}
\left(\mathbf{J}^{-1}\right)^{-1}=\mathbf{J} \\
{\left[\begin{array}{ccccc}
j_{1,1} & \cdots & \cdots & \cdots & j_{1, q} \\
\vdots & \vdots & \vdots & \vdots & \vdots \\
0 & \cdots & 0 & \cdots & 1_{k, q} \\
\vdots & \vdots & \vdots & \vdots & \vdots \\
j_{p, 1} & \cdots & \cdots & \cdots & j_{p, q}
\end{array}\right]_{(\mathrm{p}, \mathrm{q})}^{-1}=\left[\begin{array}{ccccc}
j_{1,1} & \cdots & \cdots & \cdots & j_{1, p} \\
\vdots & \vdots & \vdots & \vdots & \vdots \\
\vdots & \vdots & \vdots & \vdots & \vdots \\
j_{q-1,1} & \vdots & \vdots & \vdots & \vdots \\
0 & \cdots & 1_{q, k} & \cdots & 0
\end{array}\right]_{(\mathrm{q}, \mathrm{p})}}
\end{gathered}
$$

Observa-se que a posição do parâmetro de continuação é alterada por uma simples "transposição".

Esta nova matriz Jacobiana influi diretamente no cálculo do vetor tangente. Como visto anteriormente, havia um obstáculo a ser superado no que dizia respeito ao fato de o número de equações ser inferior ao número de incógnitas. Solucionado o impasse, o cálculo do vetor tangente é feito agora, como mostrado a seguir:

$$
\left[\begin{array}{ccc}
\mathbf{F}_{\boldsymbol{\delta}} & \mathbf{F}_{\mathbf{V}} & \mathbf{F} \lambda \\
& \mathbf{e}_{k} &
\end{array}\right]\left[\begin{array}{c}
d \boldsymbol{\delta} \\
d \mathbf{V} \\
d \lambda
\end{array}\right]=\left[\begin{array}{c}
0 \\
\pm 1
\end{array}\right], \quad \text { onde } \mathbf{e}_{k}=\left[\begin{array}{lllll}
0 & \cdots & 1^{(k)} & \cdots & 0
\end{array}\right]
$$

Dois aspectos importantes estão embutidos na Equação 5.14: 
- uma escolha correta do índice $k$ garante a não singularidade da matriz Jacobiana aumentada no PMC;

- a justificativa de se ter o valor de um dos componentes do vetor tangente igual a +1 ou -1 reside no fato de que tal prática irá fornecer um sentido de variação na direção do k-ésimo componente do vetor tangente. Se a k-ésima variável sofrer um acréscimo, o valor a ser usado é +1 ; caso contrário usa-se -1 .

Tendo sido calculado o vetor tangente, dá-se um passo em sua direção:

$$
\left[\begin{array}{c}
\boldsymbol{\delta}^{*} \\
\mathbf{V}^{*} \\
\lambda^{*}
\end{array}\right]=\left[\begin{array}{c}
\boldsymbol{\delta} \\
\mathbf{V} \\
\lambda
\end{array}\right]+\sigma\left[\begin{array}{c}
d \boldsymbol{\delta} \\
d \mathbf{V} \\
d \lambda
\end{array}\right]
$$

O símbolo '*' representa a solução predita e $\sigma$ é um escalar que define o tamanho do passo. Deve-se dar atenção especial a $\sigma$, uma vez que a solução predita pode levar a uma condição de divergência, caso esta solução esteja fora do raio de convergência do método de Newton (aplicado ao passo corretor); ou a uma solução indesejada.

Finalizando o método da continuação, resta saber como verificar se o PMC foi ou não atingido. Tendo em mente que o PMC representa um ponto extremo de carregamento, onde um máximo é atingido e em seguida, ocorre um decrescimento, basta verificar a componente do vetor tangente relacionada ao parâmetro de carregamento $\lambda$. Enquanto a carga sofre acréscimos, o sinal de $d \lambda$ é positivo. Após o cálculo do PMC, o valor da carga se torna decrescente e o sinal de $d \lambda$ negativo. Esta alteração no sinal de $d \lambda$ revela que o ponto extremo foi ultrapassado. Uma simples análise identifica que $d \lambda$ é igual a zero exatamente no PMC. 


\subsection{Fluxograma do Método da Continuação Aplicado ao Problema de Fluxo de Potência}

O método da continuação aplicado ao problema de fluxo de potência é representado pelo fluxograma abaixo:

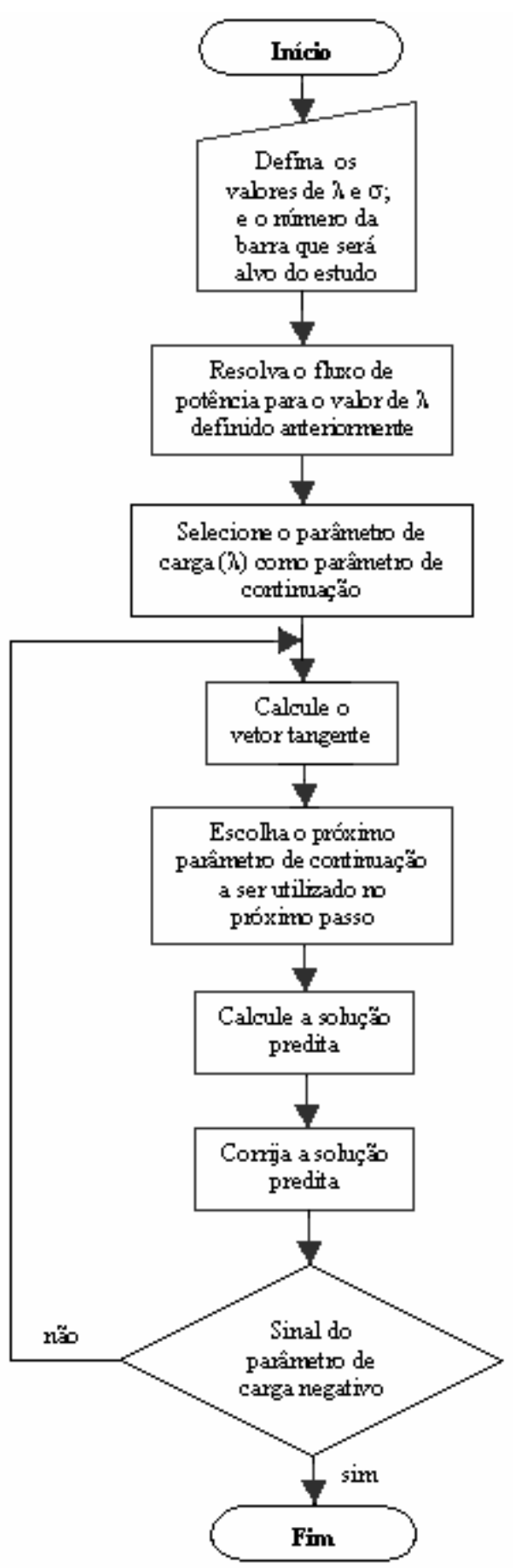

Figura 19 - Método da continuação aplicado ao problema de fluxo de potência 
Os valores definidos para $\lambda$ e $\sigma$ representam, respectivamente, o carregamento inicial do sistema em avaliação e o tamanho do passo a ser dado na direção do vetor tangente.

Por ser um valor empírico, a eficiência do processo depende de uma boa escolha de $\sigma$, uma vez que seu valor é dependente do sistema. Para obter-se um bom desempenho global, o tamanho do passo deveria se adaptar às condições reais de convergência, que, por sua vez, estará relacionada com o algoritmo em uso. Existem formas de obter-se um controle do tamanho do passo, entre elas, um método simples baseado no número de iterações do passo corretor. Enquanto este número for pequeno, têm-se condições de carga leve ou normal e o tamanho para o próximo passo poderá ser maior. Seja um número grande de iterações, o sistema se encontrará estressado e o tamanho do passo deverá ser menor. Tal método encontra uma dificuldade quando ocorre a atuação de diversos limites, desta forma, constata-se um aumento no número de iterações.

Outro método de controle de passo, usado neste trabalho, é baseado no vetor tangente normalizado. O tamanho do passo é definido como:

$$
\sigma=\frac{\sigma_{0}}{\|\mathbf{t}\|}
$$

onde $\|\mathbf{t}\|$ é a norma Euclidiana do vetor tangente, e $\sigma_{0}$ é um escalar pré-definido. Com o aumento do carregamento do sistema, a magnitude do vetor tangente aumenta e $\sigma$ diminui. Entretanto, a eficiência do processo continua dependente de uma boa escolha para $\sigma_{0}$. É de fundamental importância, para a independência do processo, encontrar um valor ótimo para $\sigma_{0}$.

\subsection{Exemplo Numérico}

O método da continuação será aplicado, com detalhes, a uma simples equação quadrática.

Considere a equação:

$$
f(x)=x^{2}-3 x
$$


A parábola mostrada a seguir revela os pontos da curva correspondentes à sua extremidade. Logo, o valor de $x$ para o ponto extremo é igual a 1,5.

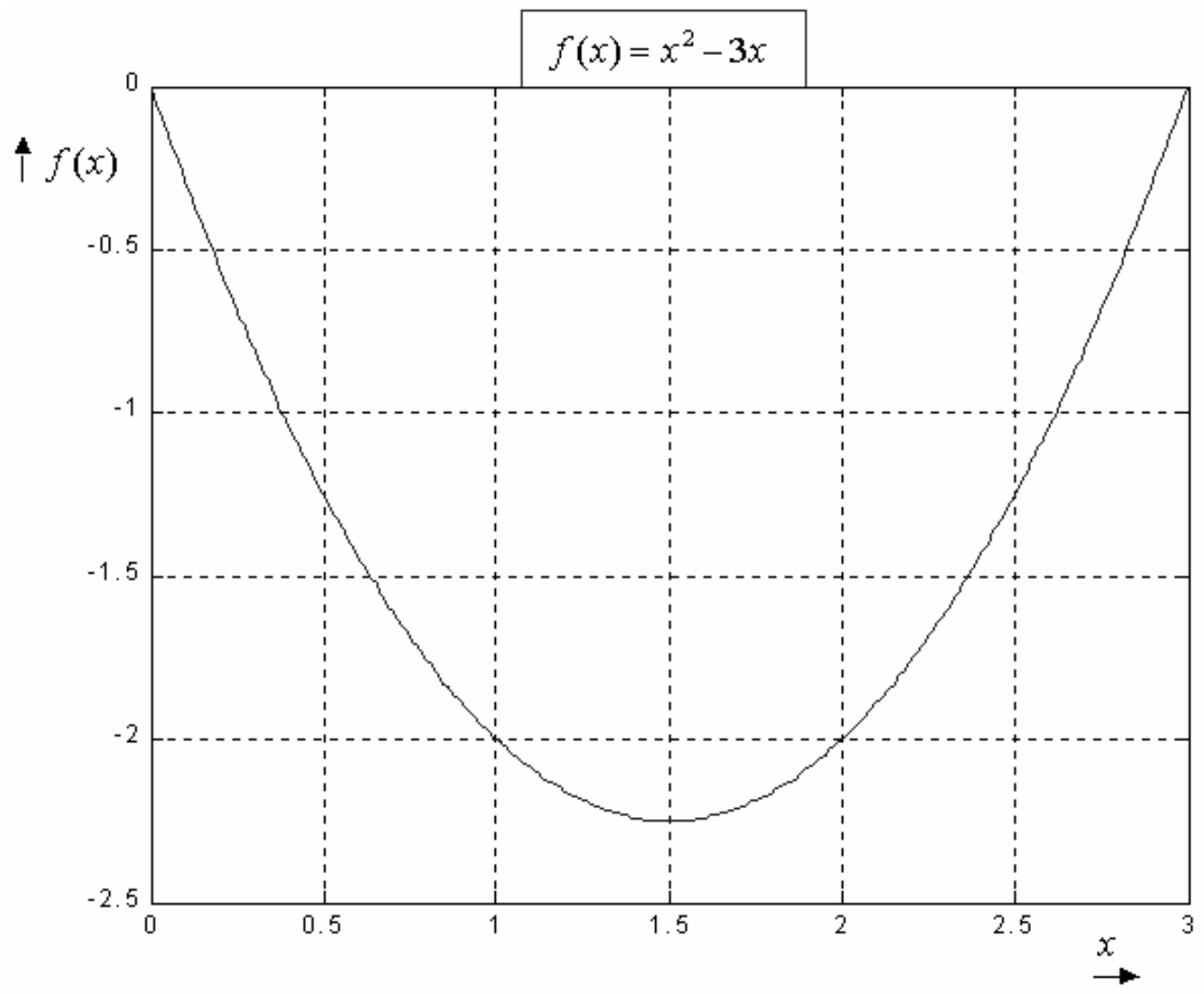

Figura 20 - Extremidade da parábola que representa $f(x)$

Com a introdução de um parâmetro, a equação em questão resulta em:

$$
x^{2}-3 x-f(x)=0 \therefore f(x, \lambda)=x^{2}-3 x+\lambda=0,
$$

onde os valores assumidos por $\lambda(\lambda=-f(x))$ são os valores negativos dos assumidos pela função $f(x)$. Então, fazendo-se variar o parâmetro $\lambda$, os valores para a variável $x$ alteram-se automaticamente.

O primeiro passo na aplicação do método da continuação é calcular o vetor tangente a uma solução que corresponda ao ponto de partida. Para isso, $\lambda$ é igualado a zero $(f(x)=0)$, fazendo com que $x$ assuma um valor igual a 3 . 
Cálculo do vetor tangente:

$$
\begin{gathered}
d f(x, \lambda)=f_{x} d x+f_{\lambda} d \lambda=0 \\
d f(x, \lambda)=(2 x-3) d x+d \lambda
\end{gathered}
$$

Escolhendo $\lambda$ como parâmetro de continuação $(k=2)$, a matriz Jacobiana pode ser construída de acordo com a Equação 5.20 (lembrando que a solução inicial é $\left.\left(x_{0}, \lambda_{0}\right)=(3,0)\right)$.

$$
\left[\begin{array}{cc}
\left(2 x_{0}-3\right) & 1 \\
0 & 1
\end{array}\right]\left[\begin{array}{l}
d x \\
d \lambda
\end{array}\right]=\left[\begin{array}{c}
0 \\
+1
\end{array}\right]
$$

Como o parâmetro de continuação inicial é uma escolha aleatória, assim também é a variação dada em sua direção. Por isso o valor +1 (variação positiva) é atribuído a $d \lambda$. Resolvendo o sistema matricial, tem-se:

$$
\left[\begin{array}{ll}
3 & 1 \\
0 & 1
\end{array}\right]\left[\begin{array}{l}
d x \\
d \lambda
\end{array}\right]=\left[\begin{array}{c}
0 \\
+1
\end{array}\right] \Rightarrow\left[\begin{array}{l}
d x \\
d \lambda
\end{array}\right]=\left[\begin{array}{c}
-1 / 3 \\
1
\end{array}\right]
$$

De imediato, é possível determinar qual será o próximo parâmetro de continuação, que seguirá sendo $\lambda$, uma vez que o componente de maior variação do vetor tangente é $d \lambda=1 ; k=2$.

\section{Cálculo da solução predita:}

$$
\left[\begin{array}{l}
x_{1}^{*} \\
\lambda_{1}^{*}
\end{array}\right]=\left[\begin{array}{l}
x_{0} \\
\lambda_{0}
\end{array}\right]+\sigma\left[\begin{array}{l}
d x \\
d \lambda
\end{array}\right]
$$

onde o tamanho do passo a ser dado será definido como $\sigma=0,5$. Logo, como resultado do passo dado na direção do vetor tangente, tem-se: $\left(x_{1}^{*}, \lambda_{1}^{*}\right)=(2,8333 ; 0,5)$.

\section{Correção da solução predita (via método de Newton):}

$$
\mathbf{g}(\mathbf{x})=-\mathbf{J}(\mathbf{x}) \Delta \mathbf{x} \therefore \Delta \mathbf{x}=-\mathbf{J}^{-1}(\mathbf{x}) \mathbf{g}(\mathbf{x}),
$$

onde:

$$
\mathbf{x}=\left[\begin{array}{l}
x \\
\lambda
\end{array}\right], \mathbf{g}(\mathbf{x})=\left[\begin{array}{c}
f(x, \lambda) \\
0
\end{array}\right] \text { e } \Delta \mathbf{x}=\left[\begin{array}{c}
\Delta x \\
\Delta \lambda
\end{array}\right]
$$


Então:

$$
\begin{gathered}
{\left[\begin{array}{c}
f\left(x_{1}^{*}, \lambda_{1}^{*}\right) \\
0
\end{array}\right]=-\left[\begin{array}{cc}
\left(2 x_{1}^{*}-3\right) & 1 \\
0 & 1
\end{array}\right]\left[\begin{array}{l}
\Delta x \\
\Delta \lambda
\end{array}\right]} \\
{\left[\begin{array}{c}
0,0277768 \\
0
\end{array}\right]=-\left[\begin{array}{cc}
2,6666 & 1 \\
0 & 1
\end{array}\right]\left[\begin{array}{l}
\Delta x \\
\Delta \lambda
\end{array}\right] \therefore\left[\begin{array}{l}
\Delta x \\
\Delta \lambda
\end{array}\right]=\left[\begin{array}{c}
-0,0104165 \\
0
\end{array}\right]}
\end{gathered}
$$

Este processo é repetido até que a solução procurada esteja dentro da faixa de convergência, ou seja, $\max \{|\Delta x|,|\Delta \lambda|\}<\varepsilon ;(\varepsilon=0,0001$, por exemplo $)$. Como isto não ocorreu, o processo continua.

$$
\begin{gathered}
x_{1 \text { novo }}^{*}=x_{1}^{*}+\Delta x=2,8229164 \\
\lambda_{1 \text { novo }}^{*}=\lambda_{1}^{*}+\Delta \lambda=0,5
\end{gathered}
$$

No momento em que a solução se encontra dentro da faixa de convergência, o processo de correção é interrompido.

$$
\begin{gathered}
{\left[\begin{array}{c}
f\left(x_{1 \text { novo }}^{*}, \lambda_{1 \text { novo }}^{*}\right) \\
0
\end{array}\right]=-\left[\begin{array}{cc}
\left(2 x_{1 \text { novo }}^{*}-3\right) & 1 \\
0 & 1
\end{array}\right]\left[\begin{array}{l}
\Delta x \\
\Delta \lambda
\end{array}\right]} \\
{\left[\begin{array}{c}
0,0001078 \\
0
\end{array}\right]=-\left[\begin{array}{cc}
2,6458328 & 1 \\
0 & 1
\end{array}\right]\left[\begin{array}{l}
\Delta x \\
\Delta \lambda
\end{array}\right] \therefore\left[\begin{array}{l}
\Delta x \\
\Delta \lambda
\end{array}\right]=\left[\begin{array}{c}
-0,000040743 \\
0
\end{array}\right]}
\end{gathered}
$$

Após o primeiro passo do método da continuação, obtém-se como solução: $\left(x_{1}, \lambda_{1}\right)=(2,8228757 ; 0,5)$. A partir deste ponto um novo vetor tangente será calculado e o processo seguirá até o seu final, onde o ponto extremo identificado terá como coordenadas: $x=1,5$ e $\lambda=2,25$, exatamente as mesmas coordenadas observadas na Figura 20.

A Figura 21 ilustra o processo da continuação e revela a importância da técnica de parametrização local. 


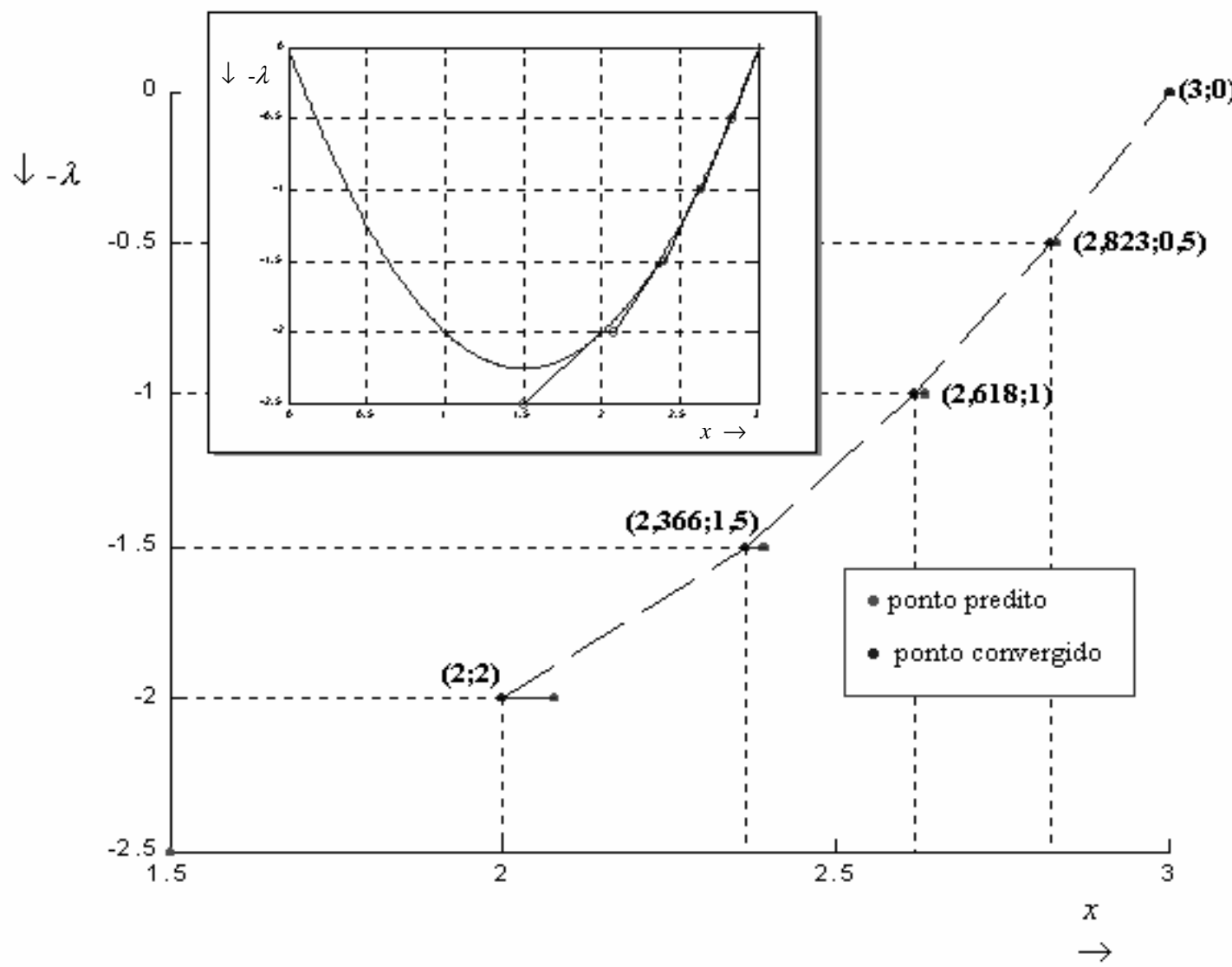

Figura 21 - Método da continuação parametrizado por $\lambda$

É interessante notar que o passo dado na direção do vetor tangente, calculado a partir do ponto de coordenadas $(2 ; 2)$, prevê uma solução cujas coordenadas são $(1,5 ; 2,5)$. Sendo $\lambda$ o parâmetro de continuação, o processo divergirá em qualquer direção que a solução caminhe. Isto se deve à singularidade da matriz Jacobiana aumentada $\left(\operatorname{det}\left(\mathbf{J}_{\text {aum }}\right)=0\right)$ no ponto extremo.

Com o emprego da parametrização local, a divergência será evitada. Comparando-se, por exemplo, as matrizes Jacobianas aumentadas para os dois possíveis parâmetros de continuação:

$$
\begin{aligned}
& \lambda \text { como parâmetro: } \mathbf{J}_{\text {aum }}=\left[\begin{array}{cc}
(2 x-3) & 1 \\
0 & 1
\end{array}\right]_{x=1,5} \Rightarrow \operatorname{det}\left(\mathbf{J}_{\text {aum }}\right)=0 \\
& x \text { como parâmetro: } \mathbf{J}_{\text {aum }}=\left[\begin{array}{cc}
(2 x-3) & 1 \\
1 & 0
\end{array}\right]_{x=1,5} \Rightarrow \operatorname{det}\left(\mathbf{J}_{\text {aum }}\right) \neq 0
\end{aligned}
$$


Observa-se que, adotando $\lambda$ como parâmetro, a matriz Jacobiana aumentada é singular no ponto extremo da curva e impossibilita a determinação exata deste nos cálculos utilizados pelo método da continuação. Logo, com a escolha de $x$ para parâmetro de continuação na extremidade da curva, obtém-se:

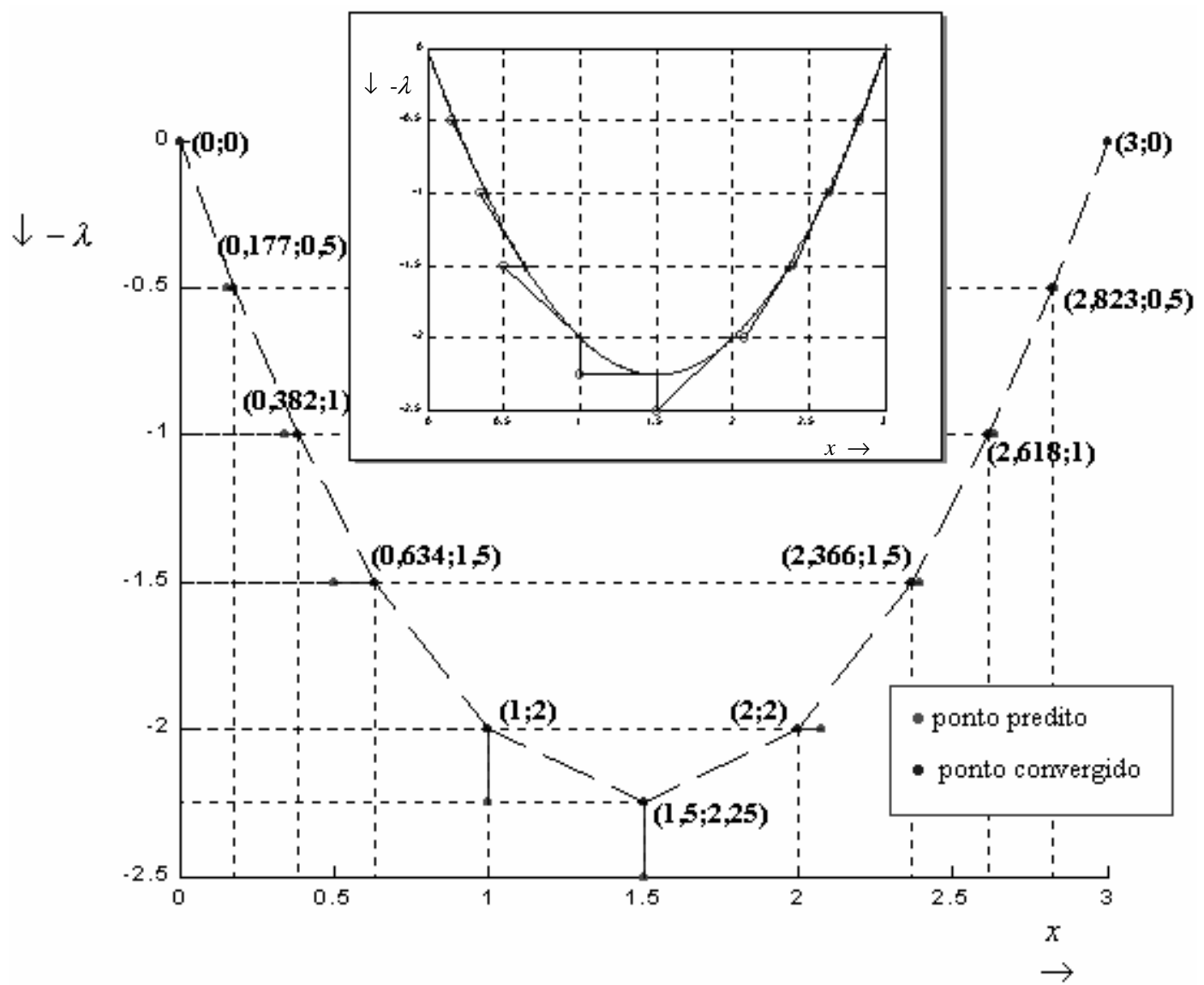

Figura 22 - Método da continuação parametrizado localmente

Os pontos de coordenadas $(1,5 ; 2,25)$ e $(1 ; 2)$ foram corrigidos considerando $x$ como parâmetro de continuação. Nota-se que, entre o ponto predito e o ponto convergido, o valor da variável $x$ não sofre alteração. Esta é a consequiência direta do emprego da técnica de parametrização, em especial, a parametrização local.

À medida que a curva é traçada, as soluções serão sempre únicas enquanto a matriz Jacobiana for não singular.

A Figura 23 mostra que, apesar de diferentes tamanhos de passos, as soluções finais sempre permanecerão sobre a trajetória de soluções. 

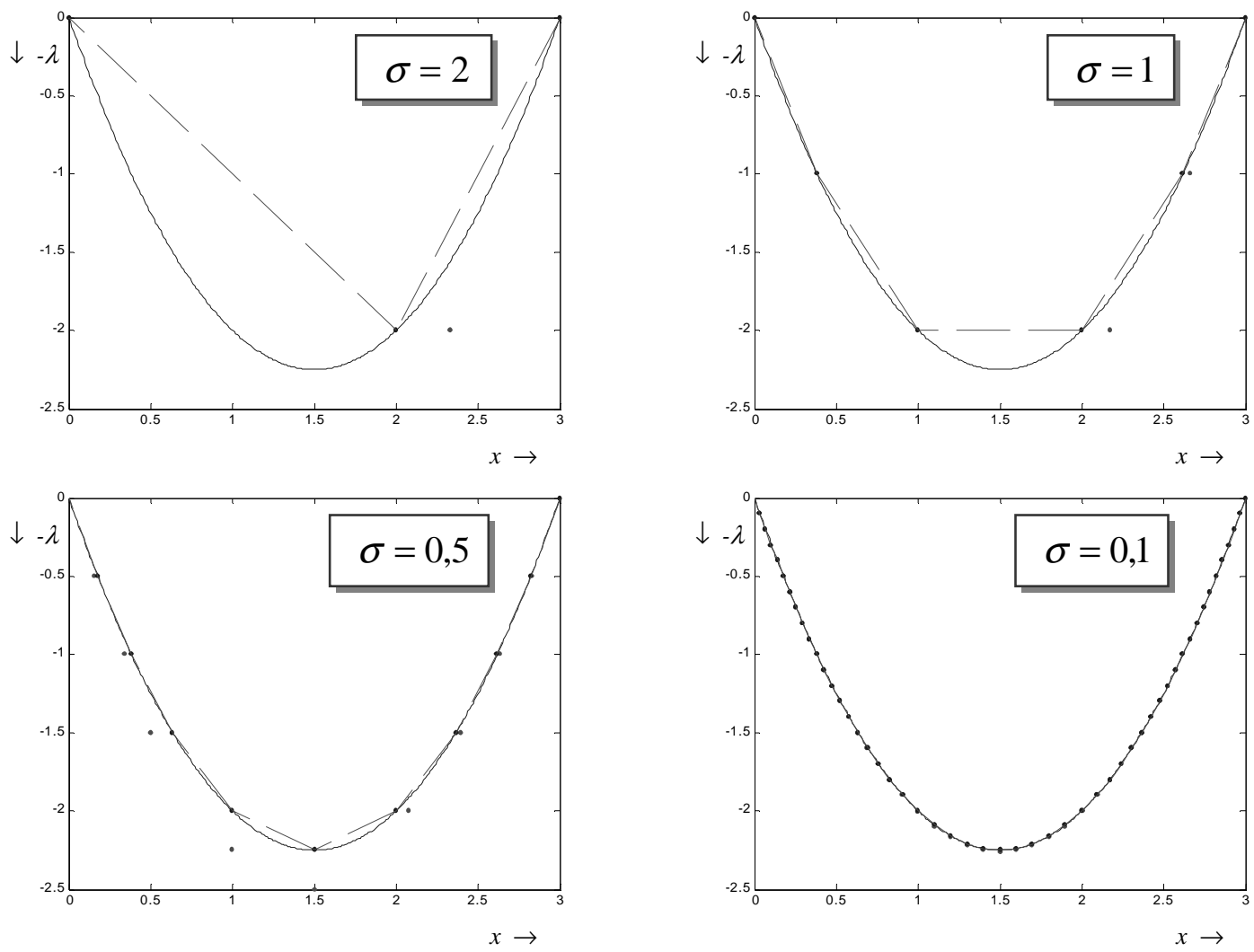

Figura 23 - Trajetória de soluções para diferentes tamanhos de passos 


\section{CAPÍTULO 6}

\section{TÉCNICA DE CONTROLE DO TAMANHO DO PASSO}

Como mencionado no item 5.4, a eficiência do processo da continuação depende de uma boa escolha de $\sigma$, uma vez que seu valor é dependente do sistema. Como a literatura disponível não apresenta estudos sobre o tamanho do passo, começou-se a estudar uma forma de abandonar este valor empírico para $\sigma$. Conseqüentemente, propôs-se uma técnica baseada no vetor tangente normalizado. Desta forma, o novo tamanho do passo é definido como:

$$
\sigma_{1}=\frac{1}{\|\mathbf{t}\|}
$$

onde $\|\mathbf{t}\|$ é a norma Euclidiana do vetor tangente. Com a proximidade do PMC, a magnitude do vetor tangente aumenta e $\sigma_{1}$ diminui.

A utilização deste novo passo acarreta algumas modificações no algoritmo correspondente ao método da continuação; isto, porém, não afeta sua estrutura original.

Assim como descrito no item 5.4, alguns artifícios foram desenvolvidos com o intuito de se fazer um controle eficaz do tamanho do passo e garantir um bom desempenho durante todo o processo. Estes artifícios são, em sua maioria, aplicados próximos ao PMC. São eles:

\section{Valor Máximo para o Passo (artifício 1)}

À primeira vista, parece correto afirmar que a magnitude do vetor tangente sempre aumentará na medida em que o método caminhar rumo ao PMC. Entretanto, esta afirmativa não é verdadeira, uma vez que o parâmetro de continuação é representado por diferentes variáveis durante todo o processo. Assim, quando $\lambda$ deixa de ser o parâmetro de continuação para dar lugar a alguma outra variável, a 
magnitude do vetor tangente pode sofrer um decréscimo. Ou seja, a magnitude do vetor tangente também depende do parâmetro de continuação.

Para evitar um aumento no valor de $\sigma_{1}$, mesmo com a aproximação do PMC, o valor do passo anterior deve ser fixado como limite máximo para o passo subseqüente.

\section{Solução de Baixa Tensão (artifício 2)}

Caso um ponto convergido se encontre sobre a porção inferior da curva PV correspondente à barra em estudo, $\sigma_{1}$ se tornará negativo. Isto é, o cálculo da margem de carregamento não será afetado mesmo com a obtenção de soluções de baixa tensão.

\section{Ponto Estimado Divergente (artifício 3)}

Outra situação que merece destaque é a que se refere a um ponto estimado, localizado fora da sua região de convergência. Quando isto ocorrer, a próxima variável que apresentar a maior taxa de variação próxima àquela solução dada, representará o novo parâmetro de continuação, indicando que o processo já estará próximo do PMC.

A partir de então, é atribuído a $\sigma_{1}$ um valor fixo igual a 0,1 (caso a solução seja de baixa tensão, o valor de $\sigma_{1}$ será igual a -0,1). A explicação da atribuição deste valor ao passo se dá na forte relação existente entre divergência e proximidade do PMC quando se emprega esta nova técnica para o controle do tamanho do passo.

\section{Amortecimento (artifício 4)}

Em alguns casos, após $\sigma_{1}$ ter assumido um valor fixo igual a $\pm 0,1$, soluções de alta e baixa tensão podem ser determinadas sucessivamente. Estes loops em torno do PMC podem interferir no desempenho do processo. Para manter a eficácia do 
controle do tamanho do passo, $\sigma_{1}$ é reduzido à metade $\left(\sigma_{1}= \pm 0,05\right)$ no instante em que o sinal de $d \lambda$ sofre uma variação (positivo $\rightarrow$ negativo ou negativo $\rightarrow$ positivo). Desta maneira, há um amortecimento dos loops, o que permite um cálculo rigoroso do PMC.

Uma vez observadas todas as circunstâncias descritas anteriormente, resta saber como verificar se o PMC foi ou não atingido. Sabendo que o PMC representa um ponto extremo de carregamento e que $d \lambda$ é igual a zero exatamente no PMC, basta verificar se o valor absoluto de $d \lambda$ é menor que uma tolerância $\varepsilon$ pré especificada como, por exemplo, $\varepsilon=0,1$.

A seguir, apresenta-se um fluxograma da técnica para o controle do tamanho do passo.

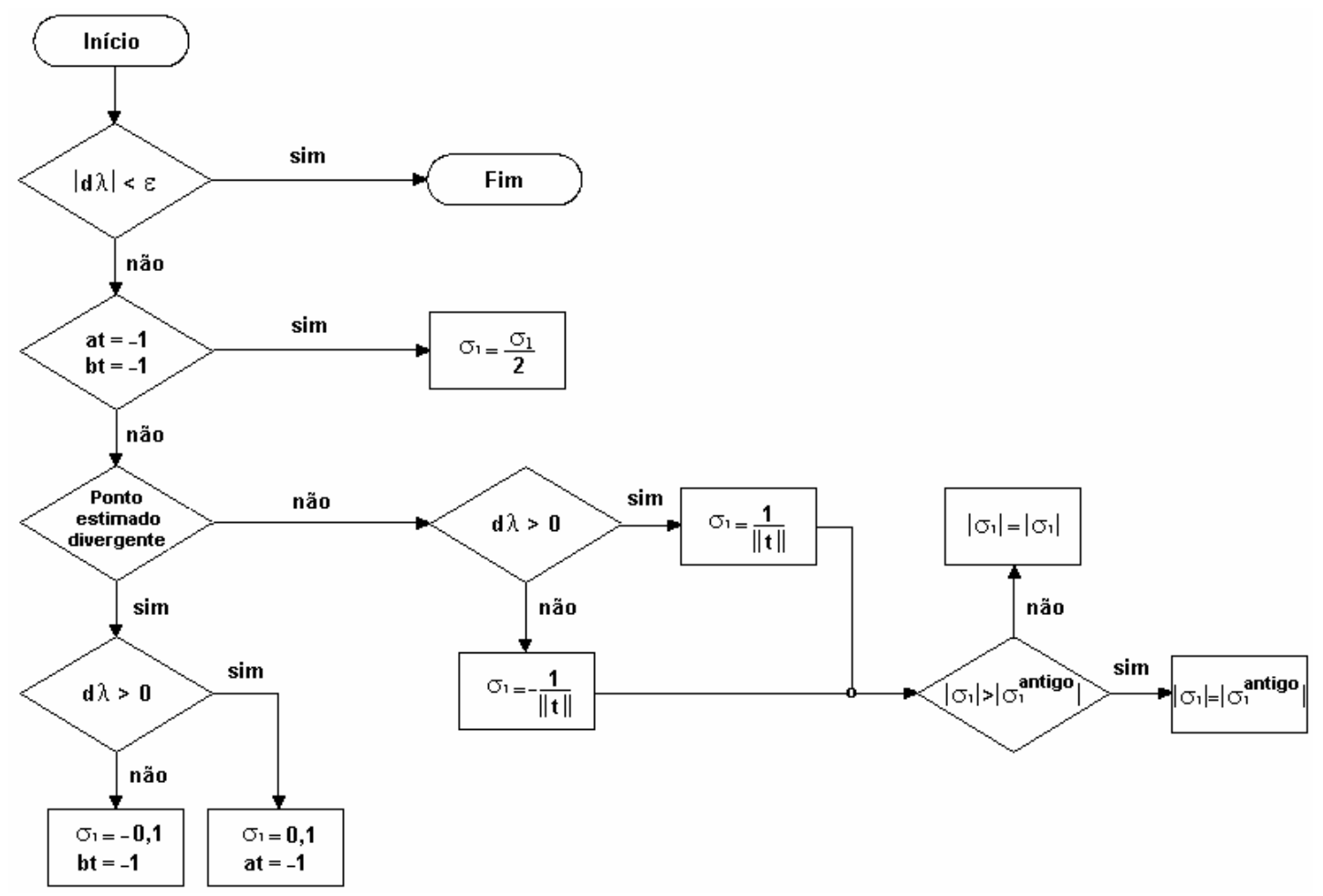

Figura 24 - Controle do tamanho do passo

A partir do instante em que houve um ponto estimado divergente, o valor -1 pode ser atribuído a $a t$ ou a $b t$. Seus valores iniciais são iguais a 0 . Caso ambos, at 
e $b t$, assumam o valor igual a -1 no decorrer do processo, o passo deve ser reduzido à metade para evitar loops em torno do PMC.

Uma característica desta técnica de controle do tamanho do passo é que, na grande maioria dos casos, ela não depende do ponto de partida. Com isto, é possível determinar o PMC a partir dos mais diversos carregamentos iniciais sem nenhuma interferência do usuário. 


\section{CAPÍTULO 7}

\section{TESTES E RESULTADOS}

Neste capítulo, serão apresentados os resultados de sistemas testes referentes à associação das equações de fluxo de potência ao método da continuação, obtidos por meio de algoritmos desenvolvidos no software MATLAB.

Serão considerados detalhamentos do processo para um sistema teste de 3 barras, ao passo que, para os demais sistemas, somente o desempenho será avaliado.

O objetivo primordial deste capítulo é, portanto, consolidar o conhecimento da teoria da continuação aplicada ao problema de fluxo de potência. Além disso, alguns resultados para validação e verificação da técnica para o controle do tamanho do passo também serão apresentados.

Para todos os testes realizados, a tolerância adotada para os mismatches foi igual a $10^{-5}$ p.u.. Em relação à $\sigma_{0}$, seus valores foram escolhidos de acordo com o sistema em análise. O primeiro ponto de cada curva foi obtido a partir do método convencional. Não será considerada a influência dos limites de tap dos transformadores, uma vez que tais limites não foram incluídos nos estudos. Em análises de colapso de tensão, deveria ser considerado um modelo que pondera os limites de corrente de campo e corrente de armadura da máquina síncrona. Contudo, o método proposto em [Cañ95-2] apud ALVES (2000), não será usado por simplicidade, isto é, as reatâncias internas dos geradores são desprezíveis e a tensão terminal é assumida como sendo constante. O modelo utilizado para as cargas é o de potência constante e o incremento de carga ativa e reativa, por meio do parâmetro $\lambda$, será feito de forma uniforme e conjunta, ou seja, mantendo o fator de potência constante do sistema. Em relação à potência gerada, somente a ativa é incrementada na mesma proporção das cargas.

$\mathrm{O}$ elemento $\mathbf{F}_{\lambda}$, da Equação 5.4, não foi mantido constante: seu valor foi recalculado a cada nova solução. Isto ocasionou uma alteração na direção, até então, fornecida pelo vetor tangente. Esta nova direção é, agora, fornecida por um vetor 
denominado vetor direção. Como conseqüência disto, os pontos estimados, em sua quase totalidade, se encontram no interior da curva PV quando sua porção superior está sendo traçada.

Seja o diagrama unifilar do sistema de três barras mostrado a seguir:

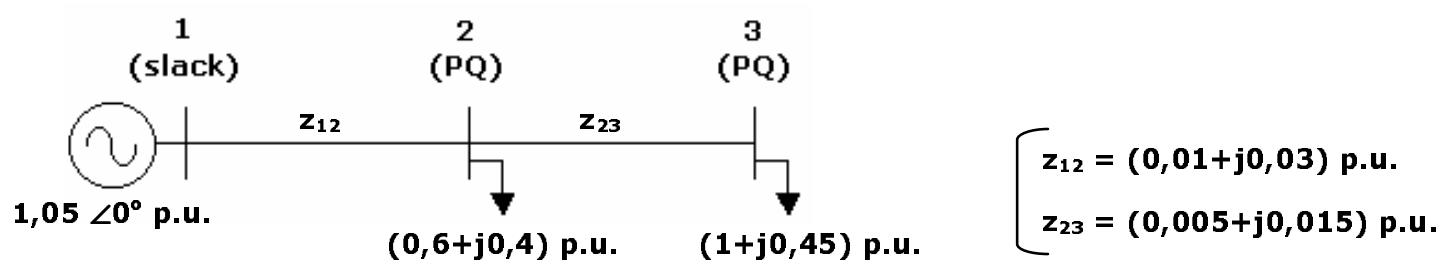

Figura 25 - Diagrama unifilar de um sistema de três barras

A curva $\lambda \mathrm{V}$, para a barra 3 e $\sigma_{0}=0,1$, plotada a partir do método da continuação, é:

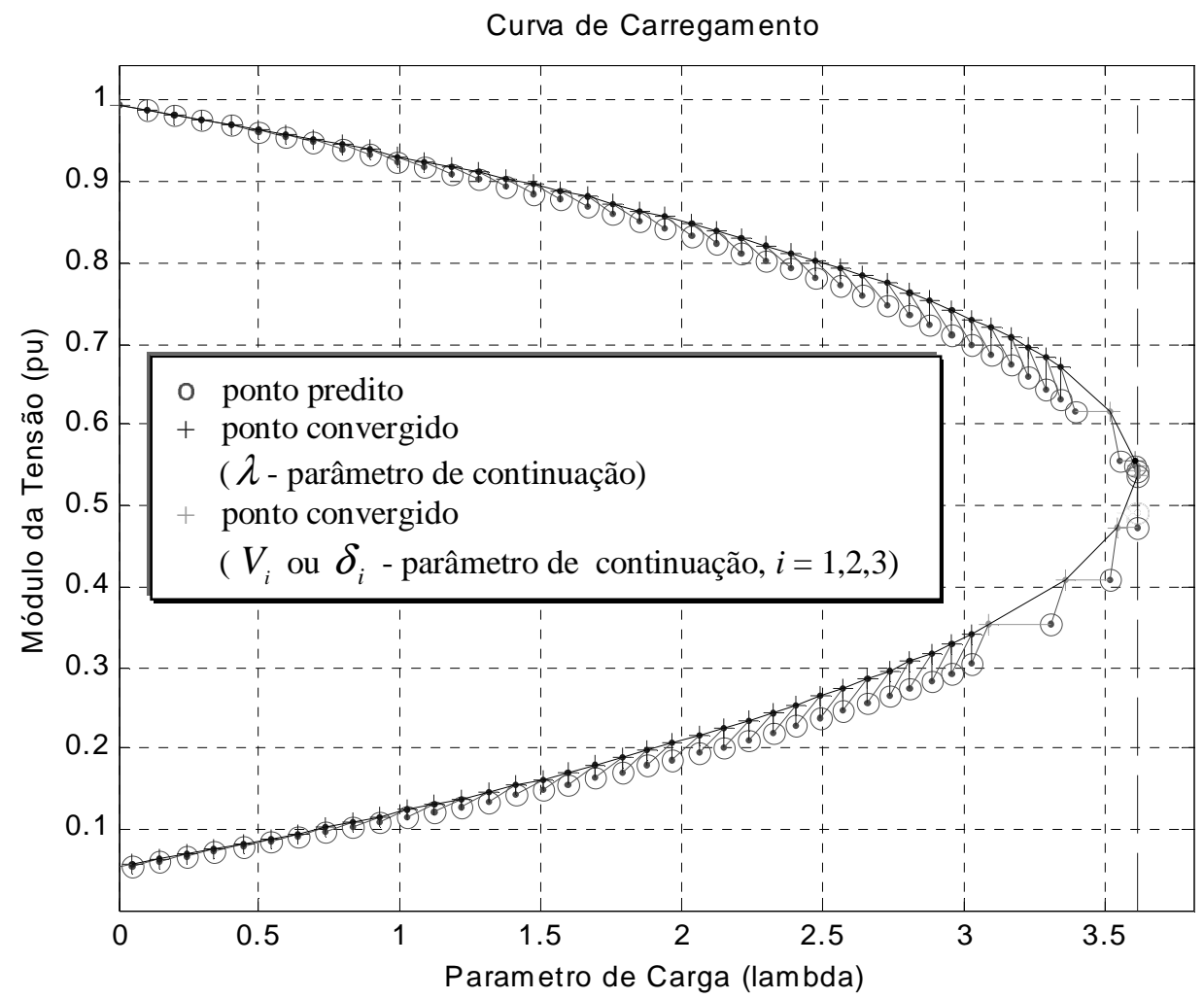

Figura 26 - Curva de carregamento para a barra 3

Desde o início do traçado da curva até as proximidades de seu ponto extremo, a variável utilizada como parâmetro de continuação foi $\lambda$. Faz-se descarte da variável $\lambda$ como parâmetro de continuação para evitar a singularidade da matriz Jacobiana 
aumentada na região próxima ao PMC. Logo, a variável adotada como parâmetro de continuação foi a magnitude da tensão na barra $3\left(V_{3}\right)$, uma vez que tal magnitude corresponde ao componente do vetor direção de maior variação durante todo o traçado da extremidade da curva. A Figura 27 apresenta a extremidade da curva ampliada para se verificar como é realizada a correção dos valores preditos para uma solução convergida adotando $V_{3}$ como parâmetro de continuação.

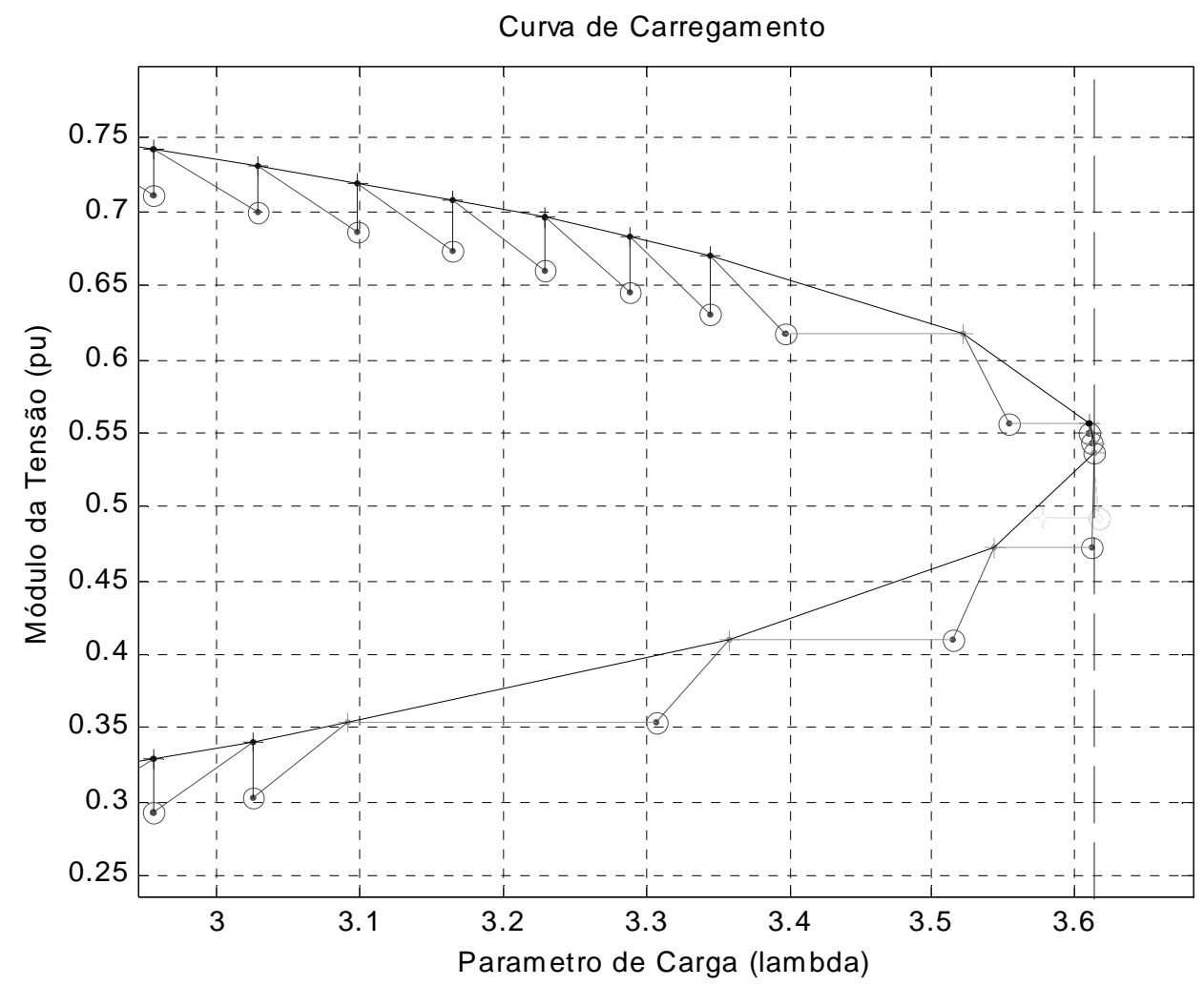

Figura 27 - Correção dos valores preditos

O método de controle de passo, baseado no vetor direção normalizado, age na extremidade da curva de forma a reduzir o passo na direção do vetor direção. Além disso, quando o PMC é determinado pela alteração do sinal de $d \lambda$, o algoritmo obriga o processo a retornar ao ponto anterior à mudança de sinal e reduz o tamanho do passo em $10 \%$ (valor adotado no algoritmo) do seu valor original. Desta forma, tem-se uma precisão maior para a determinação do PMC.

Para este sistema, o PMC ocorreu para $\lambda=3,61286$, lembrando que $\lambda=0$ corresponde ao caso base. A barra que possui a menor magnitude de tensão para todo 
o sistema, no PMC, é a barra 3. O sistema apresenta as seguintes características no PMC:

Tabela 1 - Características do sistema de 3 barras no PMC

\begin{tabular}{|c|c|r|r|r|}
\hline Barra & V (p.u.) & $\boldsymbol{\delta}$ (graus) & $\begin{array}{r}\text { Carga (MW) } \\
\text { inicial / final }\end{array}$ & $\begin{array}{r}\text { Carga (MVAr) } \\
\text { inicial / final }\end{array}$ \\
\hline 1 & 1,05 & 0 & $0 / 0$ & $0 / 0$ \\
\hline 2 & 0,6524 & $-15,43$ & $60 / 276,77$ & $40 / 184,51$ \\
\hline 3 & 0,5437 & $-24,97$ & $100 / 461,29$ & $45 / 207,58$ \\
\hline
\end{tabular}

Em relação ao ângulo de fase da barra 3, o gráfico a seguir revela seu comportamento durante o carregamento do sistema.

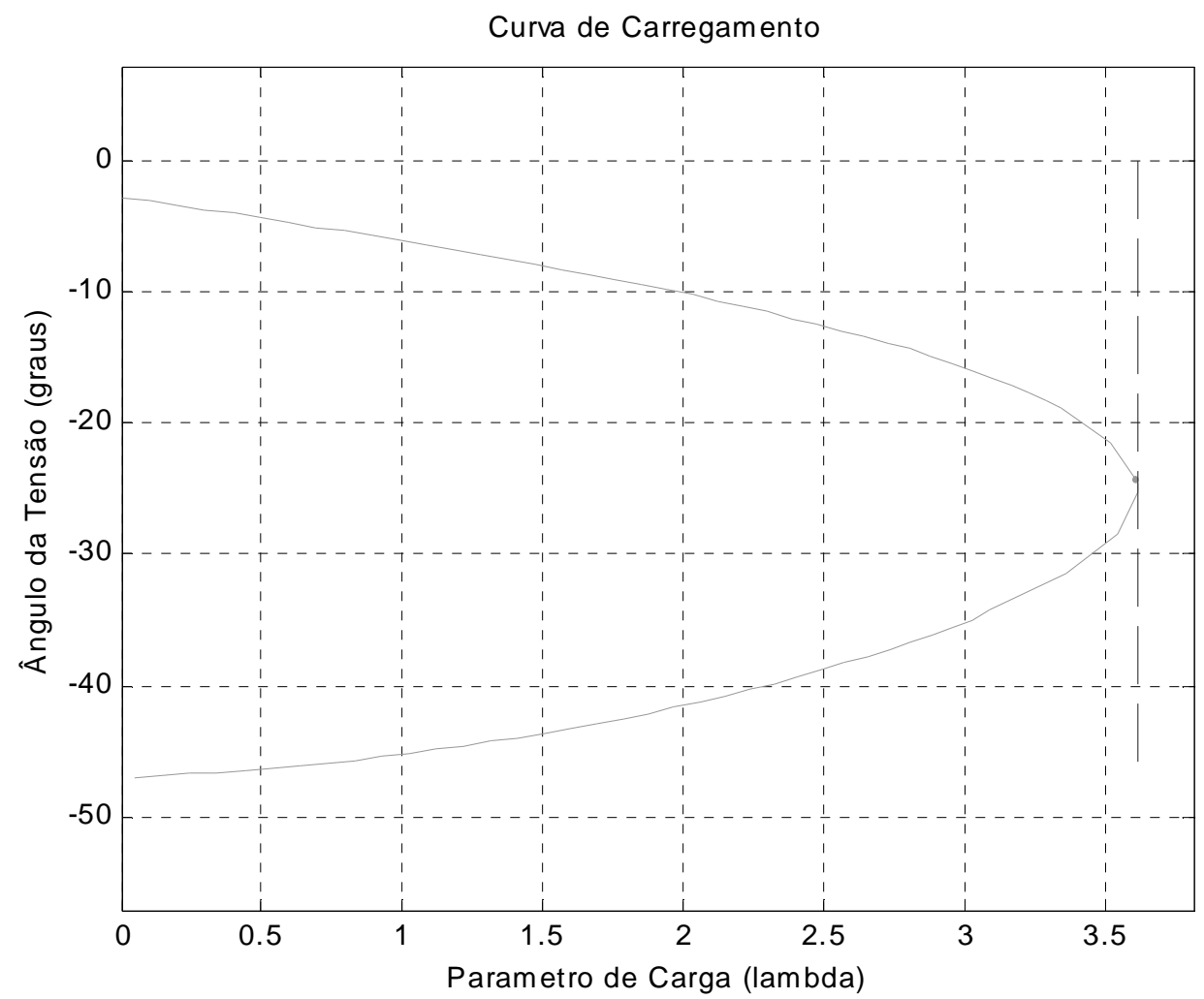

Figura 28 - Comportamento do ângulo de fase da barra 3 durante o carregamento

A mesma análise será realizada para outros sistemas testes somente com o intuito de validar a metodologia estudada neste trabalho. O tempo computacional não 
foi levado em consideração na avaliação dos resultados, uma vez que o algoritmo foi desenvolvido no software MATLAB. Entretanto, pode ser feita uma implementação adequada em linguagens de alto nível ( $\mathrm{C}++$, por exemplo), garantindo, assim, rapidez aos cálculos a serem executados, não importando a dimensão do problema.

A seguir, uma sequiência de resultados será mostrada para os demais sistemas avaliados.

\section{- IEEE-14}

Tabela 2 - Características do sistema IEEE-14 barras no PMC

\begin{tabular}{|c|c|c|c|c|}
\hline \multirow{2}{*}{ Barra } & \multicolumn{2}{|c|}{ Sem limites de reativos } & \multicolumn{2}{|c|}{ Com limites de reativos } \\
\hline & $\mathbf{V}(\mathbf{p} \cdot \mathbf{u} \cdot)$ & $\delta$ (graus) & $\mathbf{V}(\mathbf{p} \cdot \mathbf{u} \cdot)$ & $\delta($ graus) \\
\hline 1 & 1,060 & 0 & 1,060 & 0 \\
\hline 2 & 1,045 & $-36,56$ & 0,9043 & $-9,23$ \\
\hline 3 & 1,010 & $-87,81$ & 0,7465 & $-30,97$ \\
\hline 4 & 0,7064 & $-72,45$ & 0,7596 & $-23,05$ \\
\hline 5 & 0,6832 & $-61,67$ & 0,7850 & $-18,73$ \\
\hline 6 & 1,070 & $-107,86$ & 0,7247 & $-38,17$ \\
\hline 7 & 0,8037 & $-95,63$ & 0,7268 & $-34,03$ \\
\hline 8 & 1,090 & $-95,63$ & 0,7810 & $-34,03$ \\
\hline 9 & 0,7142 & $-107,71$ & 0,6787 & $-40,61$ \\
\hline 10 & 0,7344 & $-109,75$ & 0,6659 & $-41,39$ \\
\hline 11 & 0,8819 & $-109,20$ & 0,6852 & $-40,27$ \\
\hline 12 & 0,9759 & $-111,89$ & 0,6811 & $-41,67$ \\
\hline 13 & 0,9263 & $-111,95$ & 0,6662 & $-41,98$ \\
\hline 14 & 0,6896 & $-117,76$ & 0,6177 & $-45,88$ \\
\hline
\end{tabular}

As barras 5 e 14 apresentaram as menores magnitudes de tensão quando os limites de reativos foram desconsiderados. Porém, durante quase todo o traçado da 
curva, a barra que apresentou o menor valor de magnitude de tensão foi a barra 14. A partir do momento em que $\lambda$ assumiu um valor igual a 3,06, a barra 14 deixou de ser aquela com menor magnitude de tensão dando lugar à barra 5. O PMC para este sistema, sem limites de reativos, ocorreu para $\lambda=3,06254$. Quando os limites foram considerados, o PMC ocorreu para $\lambda=0,78294$.

A figura a seguir se refere à barra 14 do sistema em avaliação, sendo $\sigma_{0}=0,1$ o valor inicial para o tamanho do passo.

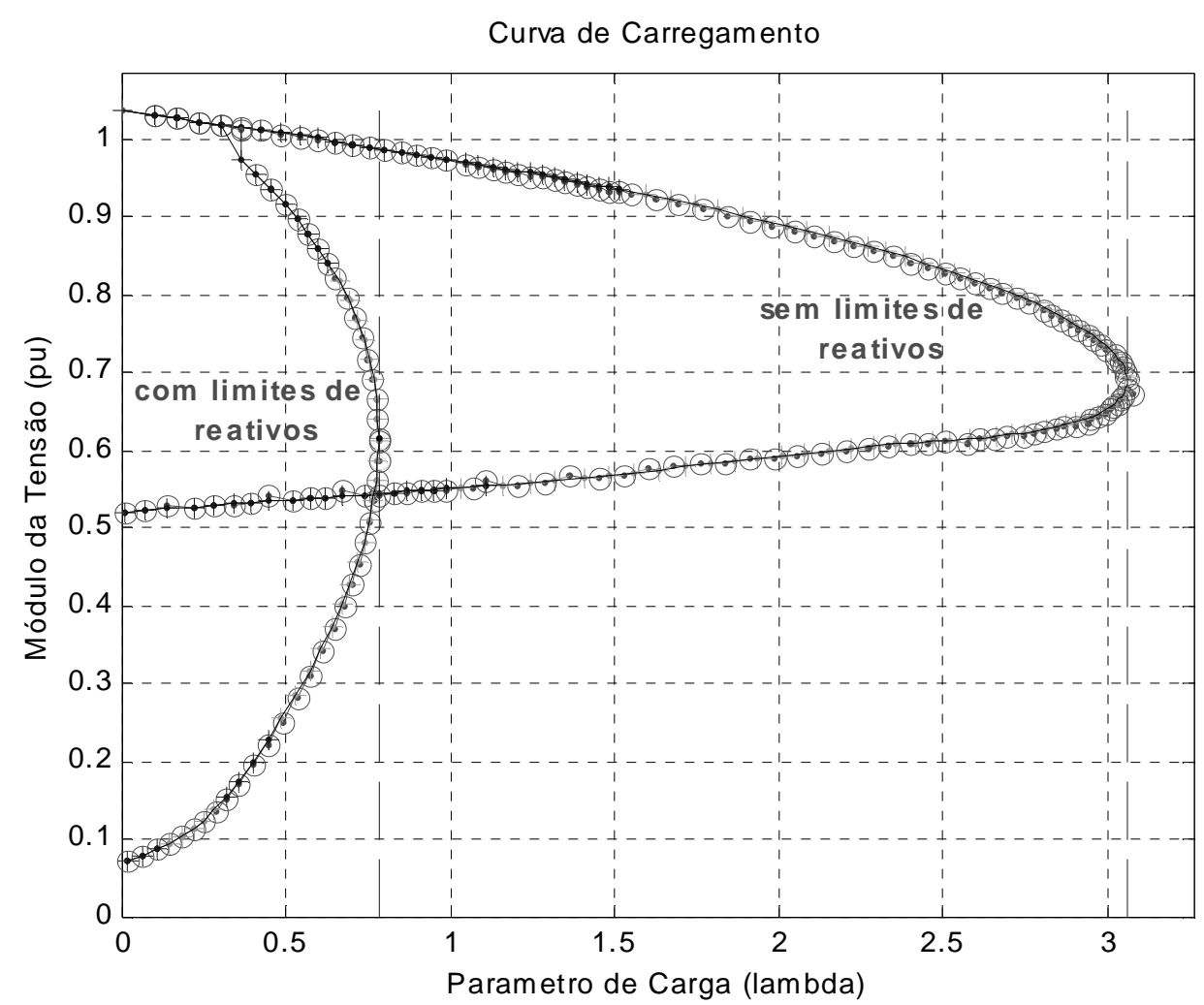

Figura 29 - Curva de carregamento, com e sem limites de reativos, para a barra 14 (IEEE-14 barras)

A Figura 29 mostra como os limites de geração de potência reativa têm grande influência no cálculo do PMC. Com base neste fato, tornam-se de grande interesse, a determinação da quantidade de potência reativa necessária e os locais onde esta deveria ser alocada para que o sistema de potência possa ser operado no mais alto grau de confiabilidade e economia possível. 

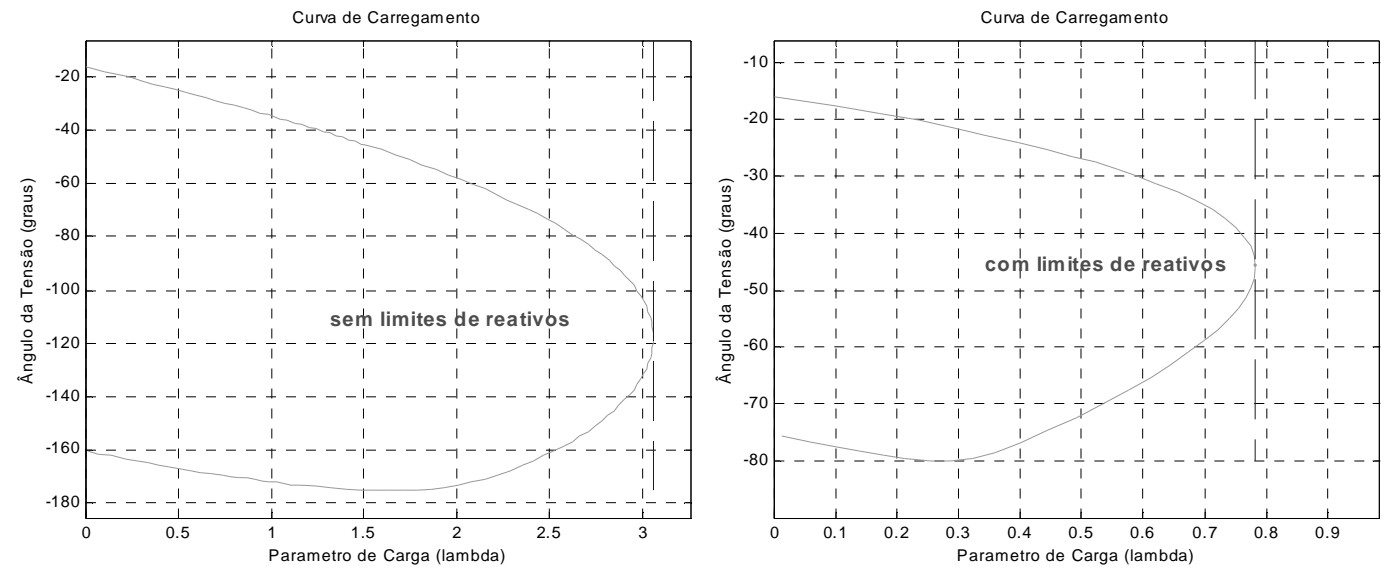

Figura 30 - Comportamento do ângulo de fase da barra 14 com e sem limites de reativos

(IEEE-14 barras)

\section{- IEEE-30}

A barra 30 apresentou a menor magnitude de tensão com a consideração ou não dos limites de reativos. Adotou-se $\sigma_{0}=0,1$. Os PMC's ocorreram para $\lambda=1,95498$ e $\lambda=0,52982$.

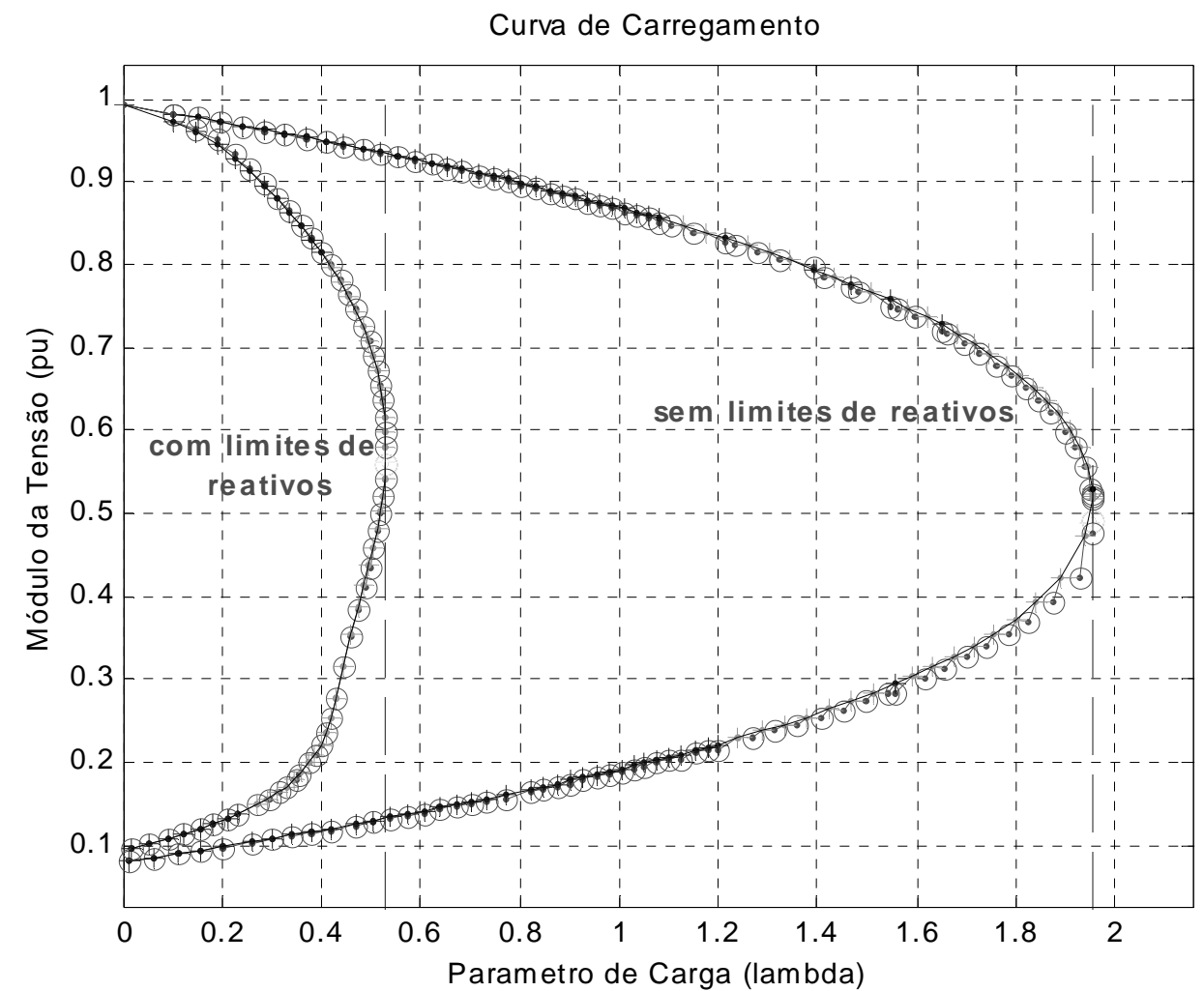

Figura 31 - Curva de carregamento, com e sem limites de reativos, para a barra 30 (IEEE-30 barras) 

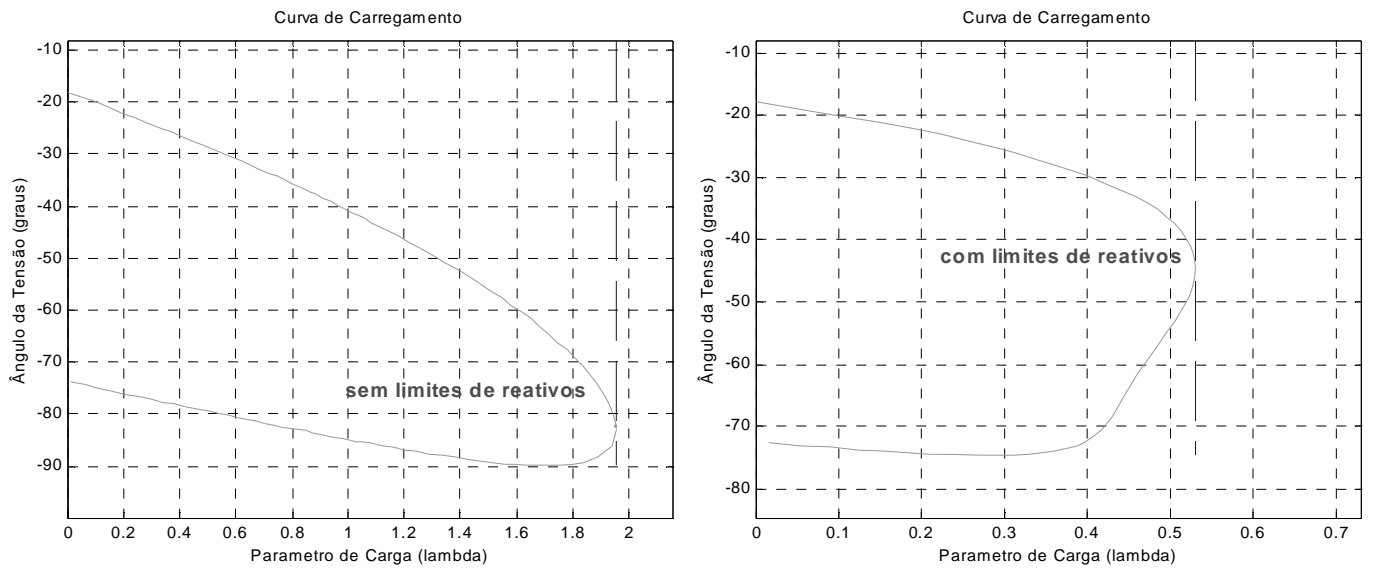

Figura 32 - Comportamento do ângulo de fase da barra 30 com e sem limites de reativos (IEEE-30 barras)

\section{- IEEE-57}

A barra 31 apresentou a menor magnitude de tensão com a consideração ou não dos limites de reativos. Adotou-se $\sigma_{0}=0,1$. Os PMC's ocorreram para $\lambda=0,89255$ e $\lambda=0,61722$.

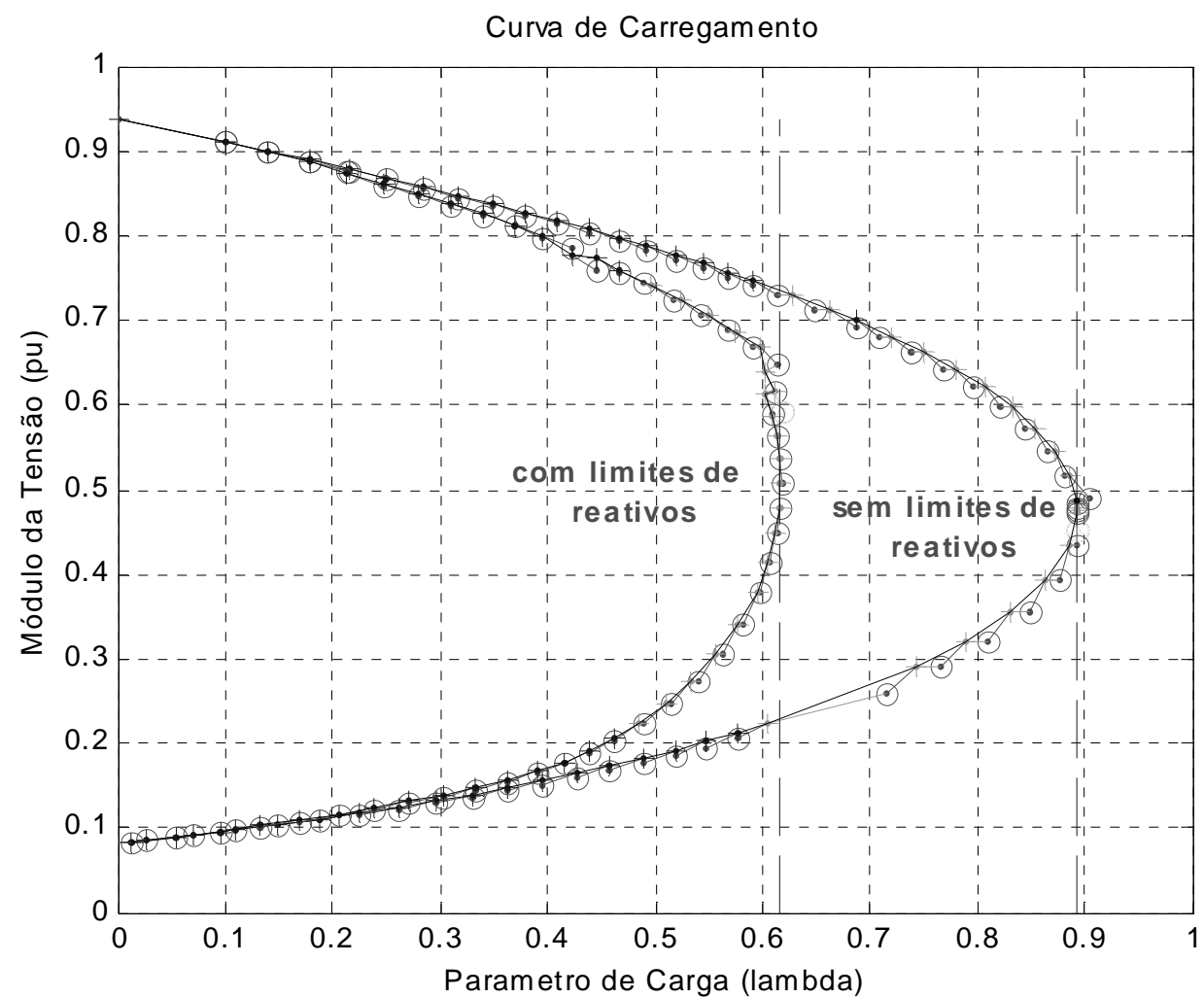

Figura 33 - Curva de carregamento, com e sem limites de reativos, para a barra 31 (IEEE-57 barras) 

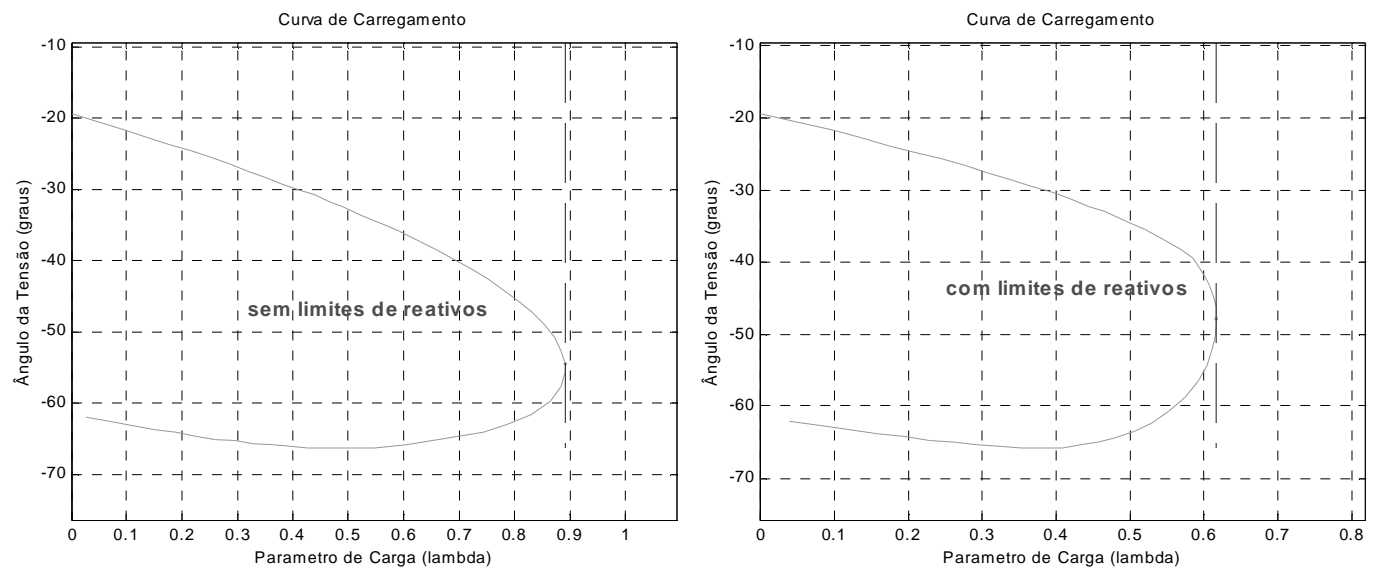

Figura 34 - Comportamento do ângulo de fase da barra 31 com e sem limites de reativos (IEEE-57 barras)

\section{- IEEE-118}

As barras 76 e 44 apresentaram as menores magnitudes de tensão quando da consideração e não consideração dos limites de geração de potência reativa, respectivamente.

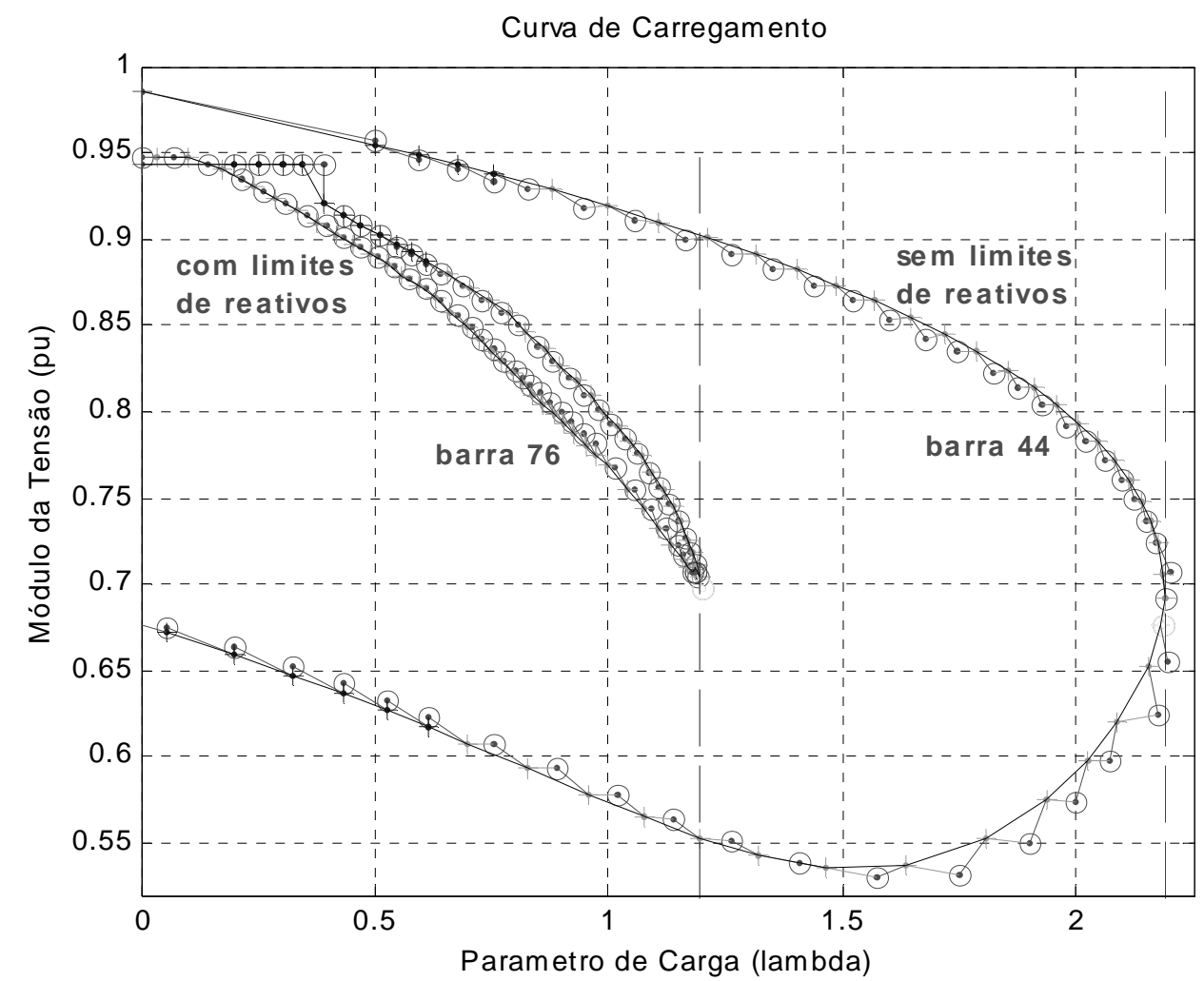

Figura 35 - Curva de carregamento, com e sem limites de reativos, para as barras 76 e 44 respectivamente (IEEE-118 barras) 
Adotou-se $\sigma_{0}=0,5$ para o traçado da curva PV referente à barra 44 e $\sigma_{0}=0,2$ para o traçado da curva PV referente à barra 76. Os PMC's ocorreram para $\lambda=2,18792$ e $\lambda=1,19550$.

Nota-se, na Figura 36, que o limite máximo de geração de potência reativa na barra 76 foi atingido. Por conseguinte, a barra 76 deixou de ser uma barra do tipo PV para se tornar uma barra do tipo PQ. A partir de então, a cada novo incremento na carga o módulo da tensão sofrerá decréscimos devido à incapacidade de se manter em um valor especificado.
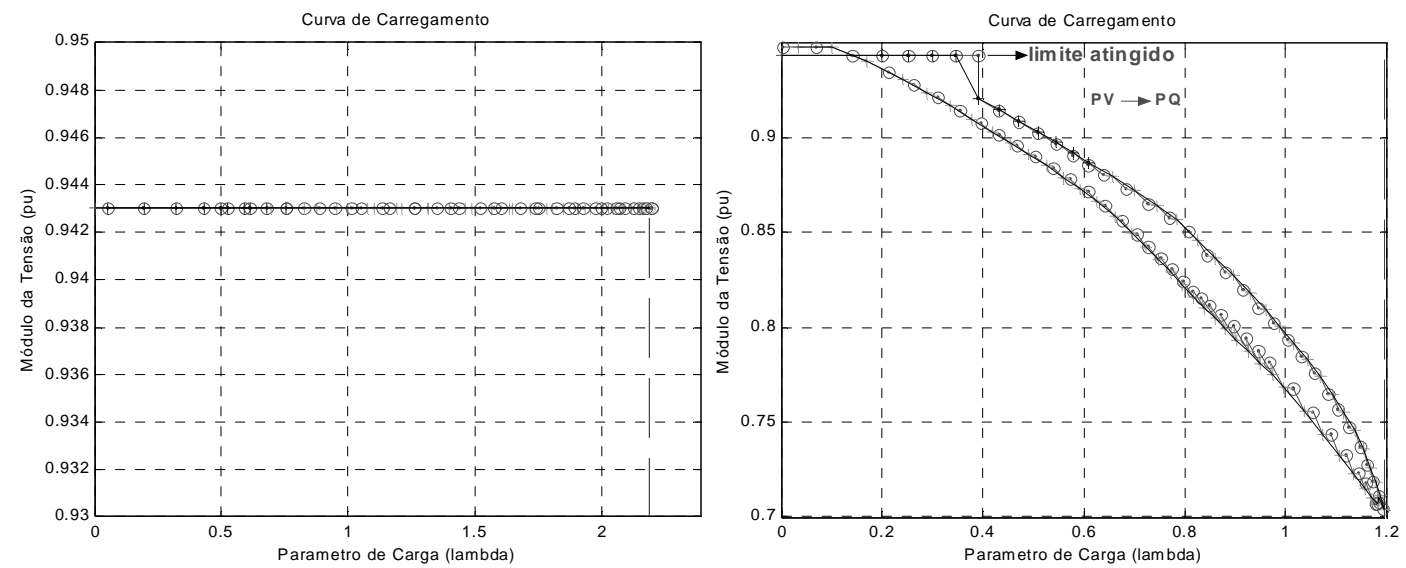

Figura 36 - Comportamento do módulo da tensão da barra 76 sem e com limites de reativos (IEEE-118 barras)

As curvas das barras 81 e 45 revelam diferentes comportamentos das tensões entre as barras quando há um carregamento. A gama de variação da magnitude da tensão na barra 81 está em torno de 0,003 p.u., ou seja, aproximadamente $8.000 \%$ a menos do que a mesma variação na barra 45 .
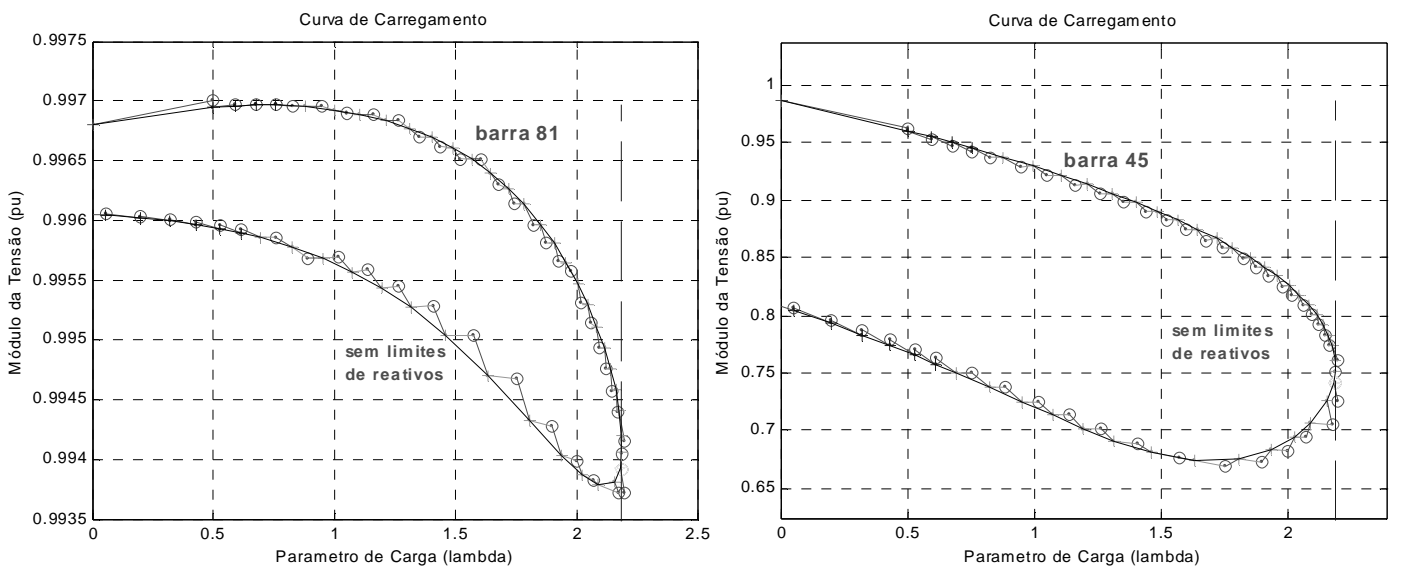

Figura 37 - Curva de carregamento para as barras 45 e 81 (IEEE-118 barras) 
Uma vez conhecido o valor do parâmetro de carga $\lambda$ no PMC, considerandose ou não os limites de geração de potência reativa nas barras PV's, é possível verificar a técnica para o controle do tamanho do passo apresentada no Capítulo 6.

A Figura 38 revela que foi possível determinar o PMC sem a necessidade de estipular um valor inicial para o tamanho do passo. Também observa-se que o resultado final não foi afetado, apesar de uma solução de baixa tensão ter sido calculada. Quando isto acontece, a direção é alterada imediatamente e, enquanto $|d \lambda|>\varepsilon$, dá-se continuidade ao processo.
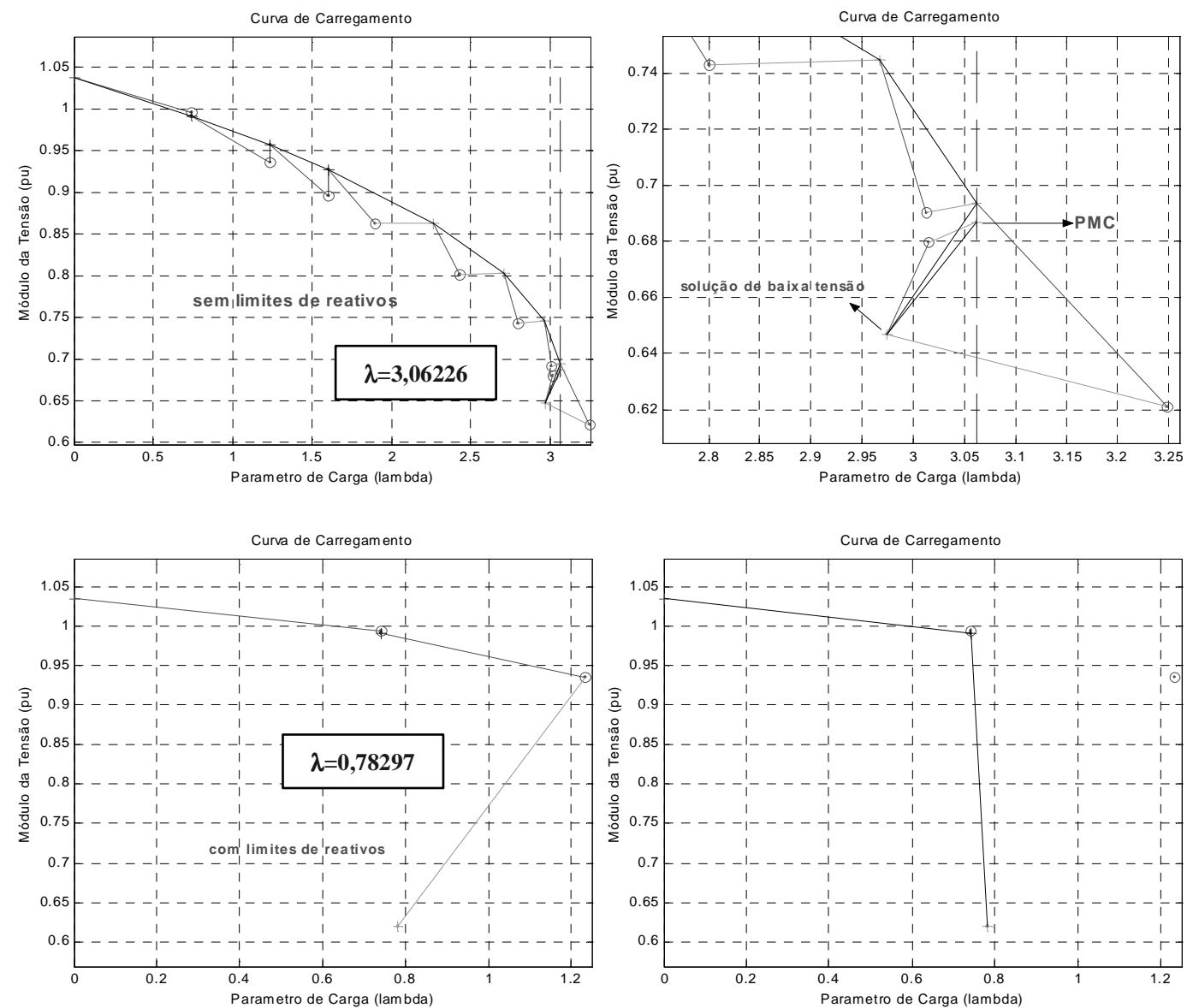

Figura 38 - Cálculo do PMC via controle de passo (barra 14; IEEE-14 barras) 
Na Figura 39, mais especificamente no cálculo do PMC quando se consideram limites, o segundo ponto estimado do processo foi um ponto divergente, uma vez que $\lambda$ era o parâmetro de continuação. Conseqüentemente, tomou-se a próxima componente do vetor direção com maior taxa de variação. Assim, determinou-se um ponto convergido. A partir deste ponto, $\sigma_{1}$ assumiu um valor, em módulo, igual a 0,1. A direção de caminhada rumo ao PMC foi determinada de acordo com a localização da solução atual: uma solução de alta tensão significa $\sigma_{1}=0,1$; e uma solução de baixa tensão, $\sigma_{1}=-0,1$. Por fim, como uma solução de baixa tensão foi encontrada após o cálculo de uma solução de alta tensão, fez-se necessário aplicar um amortecimento ( $\sigma_{1}$ foi dividido à metade) ao processo com o intuito de evitar loops em torno do PMC.
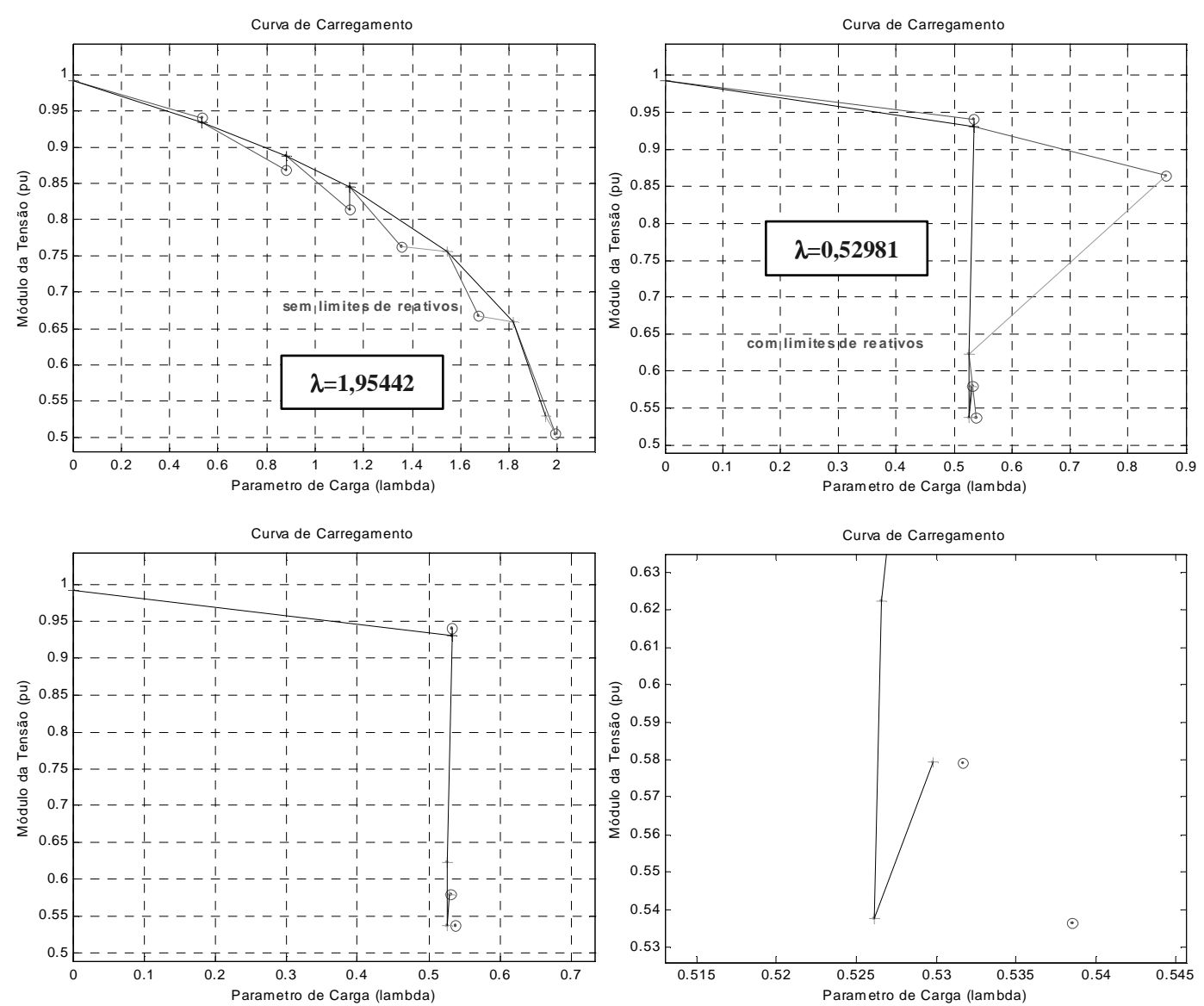

Figura 39 - Cálculo do PMC via controle de passo (barra 30; IEEE-30 barras) 

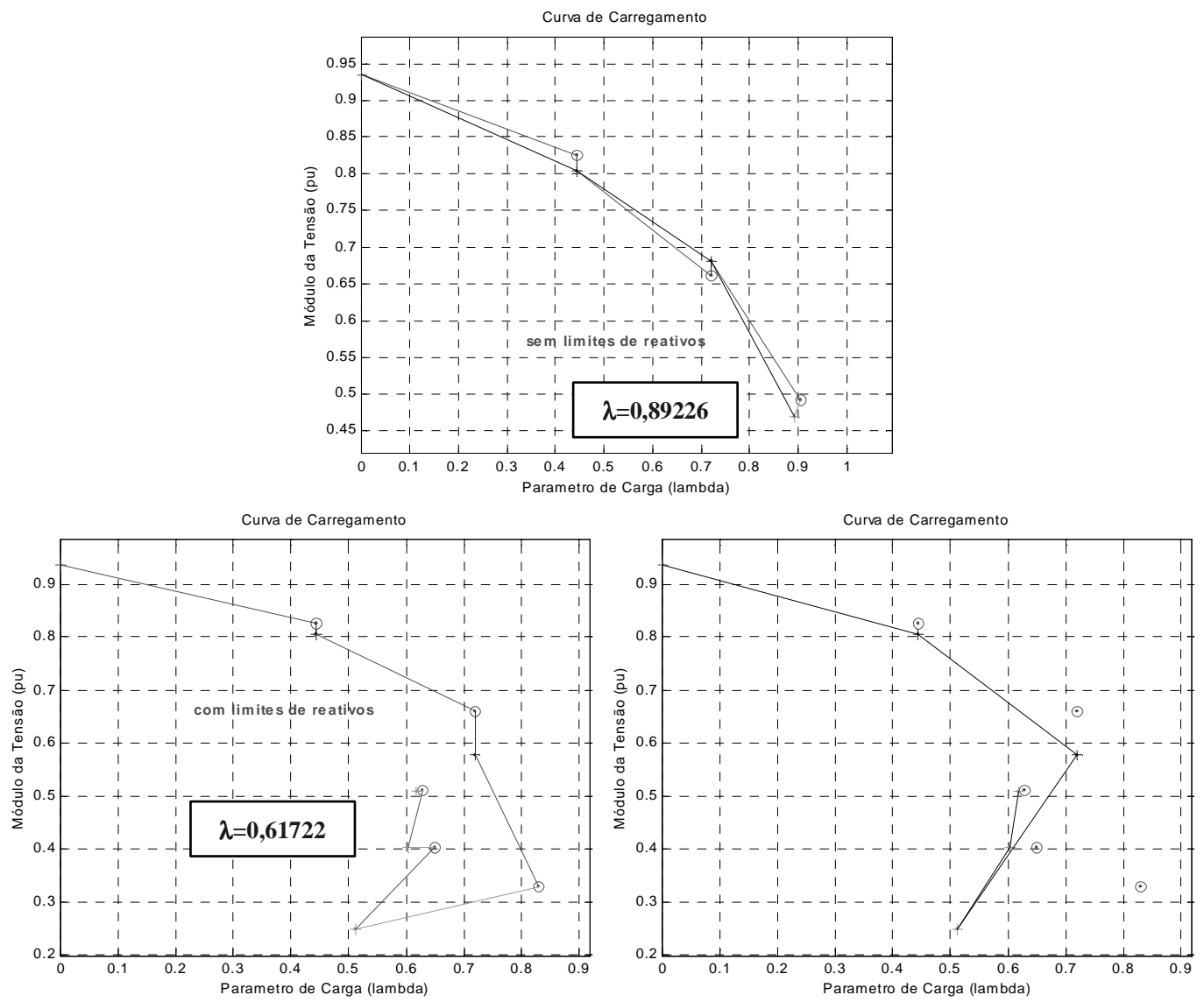

Figura 40 - Cálculo do PMC via controle de passo (barra 31; IEEE-57 barras)

Sobre a Figura 40, considerando-se a atuação dos limites de reativos, alguns comentários são extremamente importantes. O primeiro ponto convergido é um ponto pertencente à curva $\mathrm{PV}$, da barra 31 , sem a atuação dos limites de reativos. $\mathrm{O}$ segundo ponto, porém, não pertence a nenhuma das curvas mostradas na Figura 33. Este fato confirma a dependência do processo iterativo em relação à atuação dos controles existentes em um sistema. São eles que determinam a convergência do processo como um todo. $\mathrm{E}$ isto será mais verdadeiro quanto maior o número e a variedade de controles e limites representados. Logo, o efeito dos dispositivos de controle e os limites de operação só devem ser incorporados ao processo iterativo após ter sido obtida uma convergência parcial do sistema em questão. Desta forma, evitam-se problemas como a atuação indevida de dispositivos de controle e violações de limites motivadas pela escolha de valores iniciais muito distantes do ponto solução (MONTICELLI, 1983). 
Somente os últimos três pontos convergidos pertencem à curva PV plotada a partir da consideração dos limites de geração de potência reativa.

Apesar de tudo, o PMC foi determinado corretamente. Entretanto, a forma como deve ser tratada a atuação dos dispositivos de controle, em um algoritmo, influencia diretamente os resultados obtidos. Ou seja, esta é a maior dificuldade na aplicação da técnica de controle do tamanho do passo desenvolvida neste trabalho.

Na Figura 41, são apresentados os resultados para a barra 44 do sistema de 118 barras.
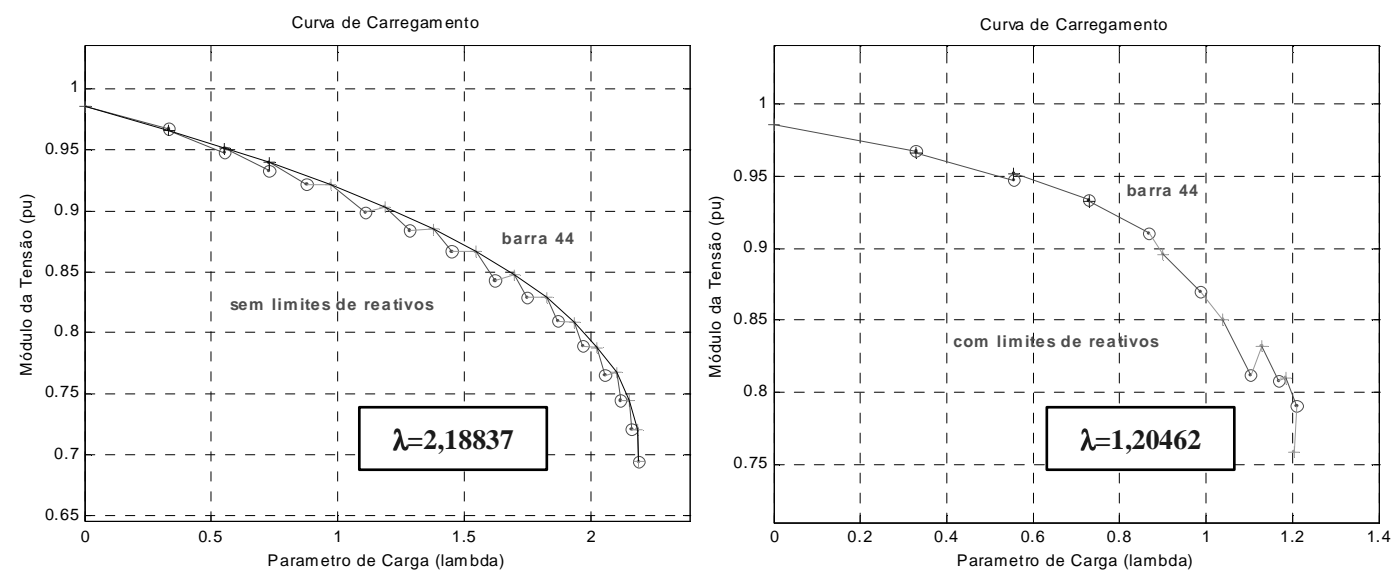

Figura 41 - Cálculo do PMC via controle de passo (barra 44; IEEE-118 barras)

As Tabelas 3, 4, 5 e 6 mostram os valores dos pontos de máximo carregamento obtidos por meio da técnica desenvolvida para o controle do tamanho do passo para diferentes carregamentos iniciais.

Tabela 3 - IEEE - 14 barras (barra 14)

\begin{tabular}{|c|c|c|c|c|}
\hline \multirow{2}{*}{$\lambda_{\mathrm{o}}$} & \multicolumn{2}{|c|}{ Sem limites de reativos $(\mathrm{PMC}=3,06254)$} & \multicolumn{2}{c|}{ Com limites de reativos (PMC = 0,78294) } \\
\cline { 2 - 5 } & PMC & erro & PMC & erro \\
\hline 0 & 3,06226 & $0,01 \%$ & 0,78297 & $0 \%$ \\
\hline 0,7 & 3,06108 & $0,05 \%$ & 0,78096 & $0,25 \%$ \\
\hline 1,4 & 3,05998 & $0,08 \%$ & ---- & - \\
\hline 2,1 & 3,02104 & $1,35 \%$ & --- & - \\
\hline
\end{tabular}


Tabela 4 - IEEE - 30 barras (barra 30)

\begin{tabular}{|c|c|c|c|c|}
\hline \multirow{2}{*}{$\lambda_{\mathrm{o}}$} & \multicolumn{2}{|c|}{ Sem limites de reativos $(\mathrm{PMC}=1,95498)$} & \multicolumn{2}{c|}{ Com limites de reativos (PMC = 0,52982) } \\
\cline { 2 - 5 } & $\mathbf{P M C}$ & erro & PMC & erro \\
\hline 0 & 1,95442 & $0,03 \%$ & 0,52981 & $0 \%$ \\
\hline 0,5 & 1,95483 & $0,01 \%$ & 0,52934 & $0,1 \%$ \\
\hline 1,0 & 1,95484 & $0,01 \%$ & ---- & - \\
\hline 1,5 & 1,95350 & $0,08 \%$ & --- & - \\
\hline
\end{tabular}

Tabela 5 - IEEE - 57 barras (barra 31)

\begin{tabular}{|c|c|c|c|c|}
\hline \multirow{2}{*}{$\lambda_{\mathrm{o}}$} & \multicolumn{2}{|c|}{ Sem limites de reativos $(\mathrm{PMC}=0,89255)$} & \multicolumn{2}{c|}{ Com limites de reativos $(\mathrm{PMC}=0,61722)$} \\
\cline { 2 - 5 } & PMC & erro & PMC & erro \\
\hline 0 & 0,89226 & $0,03 \%$ & 0,61722 & $0 \%$ \\
\hline 0,6 & 0,89234 & $0,02 \%$ & 0,61722 & $0 \%$ \\
\hline 0,8 & 0,89230 & $0,03 \%$ & --- & -- \\
\hline
\end{tabular}

Tabela 6 - IEEE - 118 barras (barra 44)

\begin{tabular}{|c|c|c|c|c|}
\hline \multirow{2}{*}{$\lambda_{\mathrm{o}}$} & \multicolumn{2}{|c|}{ Sem limites de reativos $(\mathrm{PMC}=2,18792)$} & \multicolumn{2}{|c|}{ Com limites de reativos $(\mathrm{PMC}=1,19550)$} \\
\cline { 2 - 5 } & PMC & erro & PMC & erro \\
\hline 0 & 2,18837 & $0,02 \%$ & 1,20462 & $0,76 \%$ \\
\hline 0,7 & 2,15020 & $1,72 \%$ & 1,20682 & $0,95 \%$ \\
\hline 2 & 2,16336 & $1,12 \%$ & ---- & - \\
\hline
\end{tabular}




\section{CAPÍTULO 8}

\section{CONCLUSÕES}

O método da continuação aplicado às equações de fluxo de potência e associado à técnica de parametrização local demonstrou ser capaz de evitar a singularidade da matriz Jacobiana nas proximidades do PMC. Conseqüentemente, tornou-se viável todo o traçado das curvas PV's para as mais diferentes barras de um sistema, incluindo-se o cálculo das soluções também denominadas instáveis localizadas na porção inferior destas mesmas curvas.

Um índice de proximidade do PMC pôde ser obtido com o parâmetro de carregamento $\lambda$ introduzido nas equações da rede elétrica. Uma determinação prévia do seu valor, nas condições atuais de operação de um sistema elétrico, e uma subseqüente comparação deste valor com aquele encontrado nas condições críticas revelam o quão distante do ponto de colapso de tensão se encontra o sistema avaliado. Esta "distância", ou margem de segurança, guarda uma valiosa informação capaz de fornecer, aos operadores do sistema, condições necessárias e suficientes para a realização de ações corretivas ou preventivas.

A técnica para o controle do tamanho do passo, desenvolvida neste trabalho, mostrou-se capaz de determinar o PMC sem a necessidade de especificar, por meio do usuário, um valor para o tamanho inicial do passo. Como mencionado anteriormente, a dificuldade desta metodologia está na forma como deve ser tratada a atuação dos dispositivos de controle. Devido a isto, soluções estimadas erroneamente podem ocasionar pontos convergidos que dificultam ou, até mesmo, impedem o cálculo do PMC. 


\section{BIBLIOGRAFIA CONSULTADA}

AJJARAPU, V.; CHRISTY, C. (1992). The continuation power flow: a tool for steady state voltage stability analysis. IEEE Transactions on Power Systems, v. $7, n^{\circ} 1$, p. 416-423, February.

AJJARAPU, V.; PING LIN LAU; BATTULA, S. (1994). An optimal reactive power planning strategy against voltage collapse. IEEE Transactions on Power Systems, v. 9, n 2, p. 906-917, May.

ALVES, D. A.. Obtenção do ponto de máximo carregamento em sistemas elétricos de potência utilizando novas técnicas de parametrização para o método de continuação. 2000. 116 p. Tese Doutorado - Faculdade de Engenharia Elétrica e de Computação, Universidade Estadual de Campinas, São Paulo.

ALVES, D. A.; DA COSTA, G. R. M. (2002). A geometric interpretation for transmission real losses minimization through the optimal power flow and its influence on voltage collapse. ELSEVIER Electric Power Systems Research, v. 62, n 2, p. 111-116, June.

AlveS, D. A.; DA SILVA, L. C. P.; CASTRO, C. A.; DA COSTA, V. F.. Método da continuação parametrizado pelas perdas de potência. In: CONGRESSO BRASILEIRO DE AUTOMÁTICA, 13., 2000, Florianópolis. Anais... Florianópolis: UFSC/SBC, 2000. p. 247-252.

AYASUN, S.; NWANKPA, C. O.; KWATNY, H. G. (1999). Bifurcation and Singularity analysis with voltage stability toolbox. In: NORTH AMERICAN POWER SYMPOSIUM, 31., 1999, San Luis Obispo, CA. Proceedings... San Luis Obispo: (s. ed.), 1999. p. 390-397. 
BARBOZA, L. V.; SAlGADO, R.; ALMEIDA, K. C.. Estudo do máximo carregamento de sistemas de potência via algoritmos de pontos interiores. In: CONGRESSO BRASILEIRO DE AUTOMÁTICA, 12., 1998, Uberlândia. Anais... Uberlândia: UFU, 1998. p. 2005-2010.

CAÑIZARES, C. A. (Ed.). Voltage stability assessment, procedures and guides. New York: IEEE, 2001. (IEEE/PES Power system stability subcommittee special publication). Disponível em: <http://www.power.uwaterloo.ca>. Acesso em: 25 Jan. 2002.

CAÑIZARES, C. A.; AlVARADO, F. L. (1993). Point of collapse and continuation methods for large ac/dc systems. IEEE Transactions on Power Systems, v. 8, n 1, p. 1-8, February.

CAÑIZARES, C. A.; ZAMBRONI DE SOUZA, A. C.; QUINTANA, V. H. (1994). Improving continuation methods for tracing bifurcation diagrams in power systems. In: BULK POWER SYSTEM VOLTAGE PHENOMENA, 3., 1994, Davos, Switzerland. Proceedings... Davos: (s. ed.), 1994. p. 349-358.

CASTRO, C. A. (2002). Improved method for the calculation of power systems low voltage solutions. ELSEVIER Electrical Power and Energy Systems, v. 24, $\mathrm{n}^{\mathrm{o}}$ 6, p. 503-513, August.

CHIANG, H. D.; FLUECK, A. J.; SHAH, K. S.; BALU, N. (1995). CPFLOW: A practical tool for tracing power system steady-state stationary behavior due to load and generation variations. IEEE Transactions on Power Systems, v. $10, n^{\mathrm{o}} 2$, p. 623-634, May.

FLATABO, N.; OGNEDAL, R.; CARLSEN, T. (1990). Voltage stability condition in a power transmission system calculated by sensitivity methods. IEEE Transactions on Power Systems, v. 5, nº 4, p. 1286-1293, November. 
FLUECK, A. J.; DONDETI, J. R. (2000). A new continuation power flow tool for investigating the nonlinear effects of transmission branch parameter variations. IEEE Transactions on Power Systems, v. 15, $\mathrm{n}^{\mathrm{o}}$ 1, p. 223-227, February.

GANTMACHER, F. R. (1977). The theory of matrices. v. 1(2) New York, Chelsea Publishing Company.

GUBINA, F.; STRMCNIK, B. (1995). Voltage collapse proximity index determination using voltage phasors approach. IEEE Transactions on Power Systems, v. 10, n 2, p. 788-794, May.

HILL, D. J.; LÖF, P.-A.; ANDERSON, G. (1990). Analysis of long-term voltage stability. In: POWER SYSTEM COMPUTATION CONFERENCE, 10., 1990, Graz, Austria. Proceedings... Graz: (s. ed.), 1990. p. 1252-1259.

HUNEAULT, M.; GALIANA, F. D. (1990). An investigation of the solution to the optimal power flow problem incorporating continuation methods. IEEE Transactions on Power Systems, v. 5, n 1, p. 103-110, February.

IBA, K.; SUZUKI, H.; EGAWA, M.; WATANABE, T. (1990). A method for finding a pair of multiple load flow solutions in bulk power systems. IEEE Transactions on Power Systems, v. 5, n 2, p. 582-591, May.

IBA, K.; SUZUKI, H.; EGAWA, M.; WATANABE T. (1991). Calculation of critical loading condition with nose curve using homotopy continuation method. IEEE Transactions on Power Systems, v. 6, n 2, p. 584-593, May.

IRISARRI, G. D.; WANG, X.; TONG, J.; MOKTARI, S. (1997). Maximum loadability of power system using interior point non-linear optimization 
method. IEEE Transactions on Power Systems, v. 12, n ${ }^{\mathrm{o}} 1$, p. 162-172, February.

IWAMOTO, S.; TAMURA, Y. (1981). A load flow calculation method for illconditioned power systems. IEEE Transactions on Power Apparatus and Systems, v. PAS-100, n ${ }^{\circ} .4$, p. 1736-1742, April.

KOESSLER, R. J. (1997). Voltage instability/collapse-an overview. Proceedings of the IEE Colloquium on Voltage Collapse, $\mathrm{n}^{0}$ 101, p. 1/1-1/6, April.

KUNDUR, P.; MORISON, G. K.; GAO, B. (1992). Voltage stability evaluation using modal analysis. IEEE Transactions on Power Systems, v. 7, $\mathrm{n}^{\mathrm{o}}$ 4, p. 1529-1542, November.

KWATNY, H. G.; FISCHL, R.F.; NWANKPA, C.O. (1995). Local bifurcation in power systems: theory, computation, and application. Proceedings of the IEEE, v. 83, $\mathrm{n}^{\mathrm{o}} 11$, p. 1456-1483, November.

LEITE DA SILVA, A. M.; COUTINHO, I. P.; ZAMBRONI DE SOUZA, A. C.; PRADA, R. B.; REI, A. M.. Avaliação do risco de colapso de tensão. In: CONGRESSO BRASILEIRO DE AUTOMÁTICA, 13., 2000, Florianópolis. Anais... Florianópolis: UFSC/SBC, 2000. p. 466-471.

MONTICELLI, A. (1983). Fluxo de carga em redes de energia elétrica. São Paulo, Edgard Blücher Ltda., Cap. 5, p. 75-93.

SEMLYEN, A.; GAO, B.; JANISCHEWSKYJ, W. (1991). Calculation of the extreme loading condition of a power system for the assessment of voltage stability. IEEE Transactions on Power Systems, v. 6, $\mathrm{n}^{\mathrm{o}}$ 1, p. 307-315, February. 
SEYDEL, R. (1994). Practical bifurcation and stability analysis - From Equilibrium to Chaos. 2. ed. New York, Springer-Verlag. Cap. 4, p. 125-146.

SIMÕES COSTA, A..Dinâmica e controle de sistemas elétricos de potência. Notas de aula. Disponível em <http://www.labspot.ufsc.br/ simoes>. Acesso em: 01 Fev. 2002.

TIRANUCHIT, A.; THOMAS, R. J. (1988). A posturing strategy against voltage instabilities in electric power systems. IEEE Transactions o n Power Systems, v. 3, n 1 , p. 87-93, February.

VAN CUTSEM, T. (1991). A method to compute reactive power margins with respect to voltage collapse. IEEE Transactions on Power Systems, v. 6, $\mathrm{n}^{\mathrm{o}} 1$, p. 145-156, February.

WANG, Y.; DA SILVA, L. C. P.; WILSUN, X. (2000). Investigation of the relationship between ill-conditioned power flow and voltage collapse. IEEE Power Engineering Review, v. 20, nº 7, p. 43-45, July.

ZAMBRONI DE SOUZA, A. C.. New techniques to efficiently determine proximity to static voltage collapse. $1995.119 \mathrm{p}$. $\mathrm{PhD}$ thesis - University of Waterloo, Canada. Disponível em: <http://iliniza.uwaterloo.ca/ claudio/thesis/antonio.pdf>. Acesso em: 25 Jan. 2002.

ZAMBRONI DE SOUZA, A. C.; CAÑIZARES, C.A.; QUINTANA, V.H. (1997). New techniques to speed up voltage collapse computations using tangent vectors. IEEE Transactions on Power Systems, v. 12, n ${ }^{\circ} 3$, p. 1380-1387, August. 


\section{APÊNDICE}

\section{BANCO DE DADOS DOS SISTEMAS ELÉTRICOS}

- Banco de dados do sistema IEEE - 14 barras

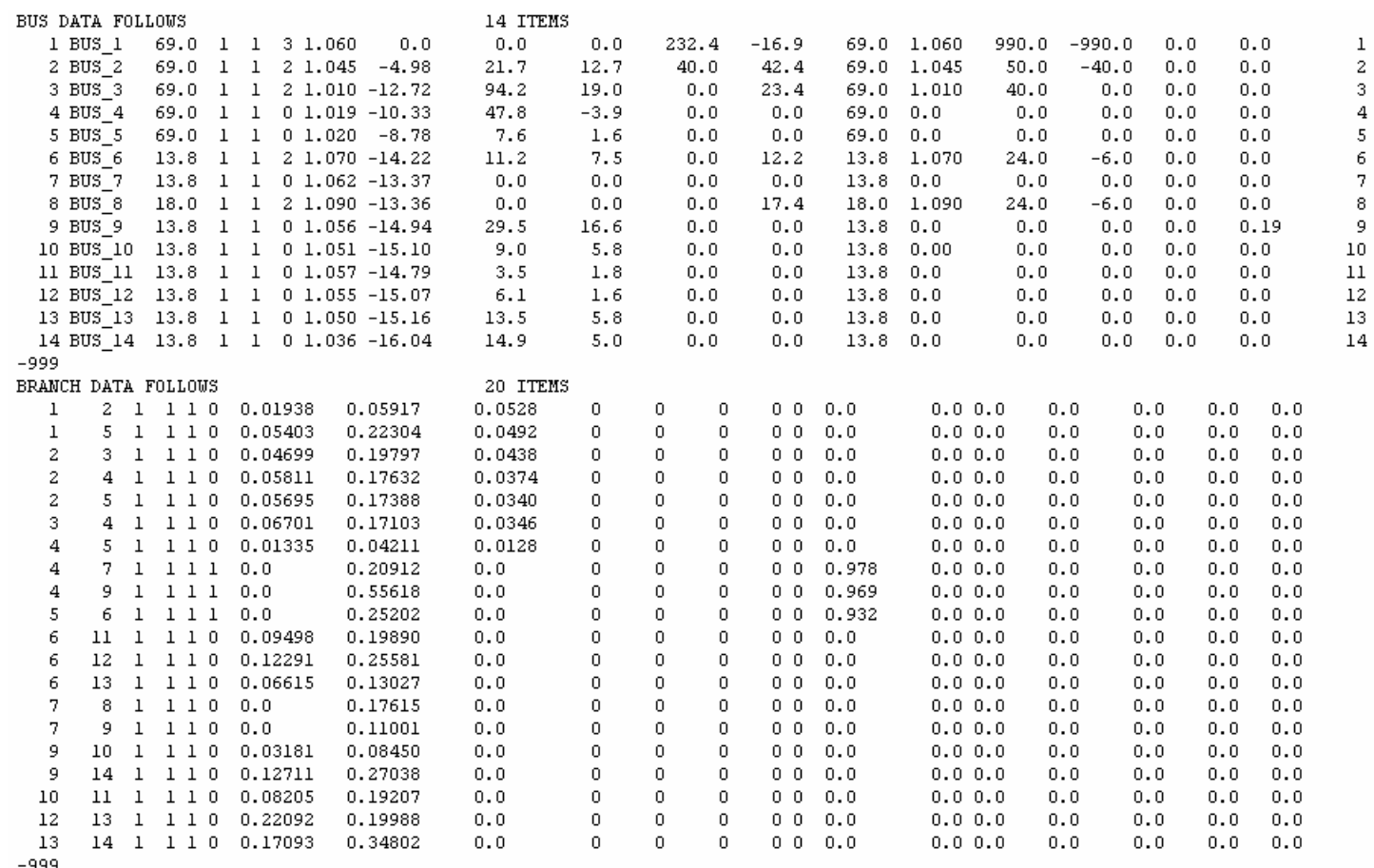




\section{- Banco de dados do sistema IEEE - 30 barras}

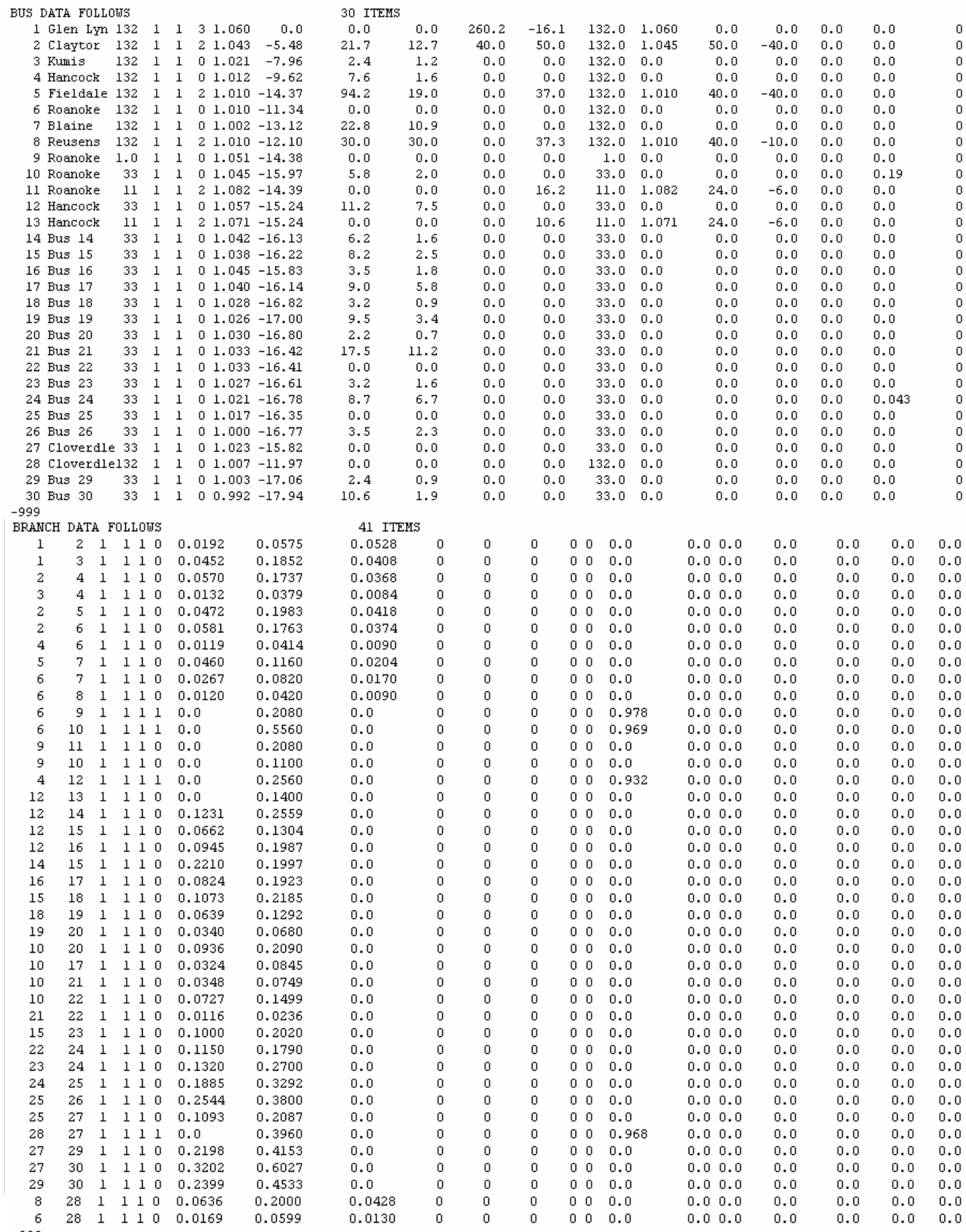


- Banco de dados do sistema IEEE - 57 barras

\begin{tabular}{|c|c|c|c|c|c|c|c|c|c|c|c|c|c|c|c|c|c|c|}
\hline EUS I & ATA $F$ & FOLLO & Dits & & & & & 57 ITEM & & & & & & & & & & \\
\hline 1 & Kanaw & wha & $\pi 1$ & 1 & 1 & 31.040 & 0.0 & 5.5 .0 & 17.0 & 128.9 & -16 & & 0.0 & 1.040 & 0.0 & 0.0 & 0.0 & 0.0 \\
\hline 2 & Tume & & ${ }_{1}$ & 1 & 1 & 21.010 & -1.18 & 3.0 & 88.0 & 0.0 & -0 & & 0.0 & 1.010 & 50.0 & -17.0 & 0.0 & 0.0 \\
\hline 3 & Logar & & ${ }_{1}$ & 1 & 1 & 20.985 & -5.97 & 41.0 & 21.0 & 40.0 & -1 & & 0.0 & 0.985 & 60.0 & -10.0 & 0.0 & 0.0 \\
\hline & Sprig & & W1 & 1 & 1 & $0 \quad 0.981$ & -7.32 & 0.0 & 0.0 & 0.0 & a. & & 0.0 & 0.0 & 0.0 & 0.0 & 0.0 & 0.0 \\
\hline & Bus 5 & & W1 & 1 & 1 & $0 \quad 0.976$ & -8.52 & 13.0 & 4.0 & 0.0 & a. & & 0.0 & 0.0 & 0.0 & 0.0 & 0.0 & 0.0 \\
\hline & Beave & rer $\mathrm{Clk}$ & $\mathrm{k}: 1$ & 1 & 1 & 20.980 & -8.65 & 75.0 & 2.0 & 0.0 & a. & & 0.0 & 0.980 & 25.0 & -8.0 & 0.0 & 0.0 \\
\hline 7 & Bus ? & & $\mathrm{W} 1$ & 1 & 1 & $0 \quad 0.984$ & $4 \quad-7.58$ & 0.0 & 0.0 & 0.0 & a. & & 0.0 & 0.0 & 0.0 & 0.0 & 0.0 & 0.0 \\
\hline 8 & Clino & ch $\mathrm{Rv}$ & $\mathrm{v} \vee 1$ & 1 & 1 & 21.005 & -4.45 & 150.0 & 22.0 & 450.0 & 62 & & 0.0 & 1.005 & 200.0 & -140.0 & 0.0 & 0.0 \\
\hline 9 & Salt & wille & e $\mathrm{V} 1$ & 1 & 1 & 20.980 & -9.56 & 121.0 & 26.0 & 0.0 & 2 & & 0.0 & 0.980 & 9.0 & -3.0 & 0.0 & 0.0 \\
\hline 10 & Bus 1 & 10 & W1 & 1 & 1 & $0 \quad 0.986$ & -11.43 & 5.0 & 2.0 & 0.0 & 0 & & 0.0 & 0.0 & 0.0 & 0.0 & 0.0 & 0.0 \\
\hline 11 & Tazen & well & $V_{1}$ & 1 & 1 & $0 \quad 0.974$ & $1-10.17$ & 0.0 & 0.0 & 0.0 & 0 & & 0.0 & 0.0 & 0.0 & 0.0 & 0.0 & 0.0 \\
\hline 12 & Glen & $\mathrm{L}_{Y M}$ & ${ }_{1}$ & 1 & 1 & 21.015 & -10.46 & 377.0 & 24.0 & 310.0 & 128 & & 0.0 & 1.015 & 155.0 & -150.0 & 0.0 & 0.0 \\
\hline 13 & Eus 1 & 13 & ${ }_{1}$ & 1 & 1 & $0 \quad 0.979$ & -9.79 & 16.0 & 2.3 & 0.0 & 0. & & 0.0 & 0.0 & 0.0 & 0.0 & 0.0 & 0.0 \\
\hline 14 & Eus 1 & 14 & ${ }_{1}$ & 1 & 1 & $0 \quad 0.970$ & -9.33 & 10.5 & 5.3 & 0.0 & 0. & & 0.0 & 0.0 & 0.0 & 0.0 & 0.0 & 0.0 \\
\hline 15 & Bus 1 & 1.5 & ${ }_{1}$ & 1 & 1 & 00.980 & -7.18 & 22.0 & 5.0 & 0.0 & 0. & & 0.0 & 0.0 & 0.0 & 0.0 & 0.0 & 0.0 \\
\hline 16 & Eus 1 & 16 & ${ }_{1}$ & 1 & 1 & 01.013 & -8.85 & 43.0 & 3.0 & 0.0 & 0. & & 0.0 & 0.0 & 0.0 & 0.0 & 0.0 & 0.0 \\
\hline 17 & Eus 1 & 17 & $\pi 1$ & 1 & 1 & 01.017 & -5.39 & 42.0 & 8.0 & 0.0 & 0. & & 0.0 & 0.0 & 0.0 & 0.0 & 0.0 & 0.0 \\
\hline 18 & Spris & $g g$ & $\pi 2$ & 1 & 1 & 01.001 & -11.71 & 27.2 & 9.8 & 0.0 & 0. & & 0.0 & 0.0 & 0.0 & 0.0 & 0.0 & 0.10 \\
\hline 19 & Eus 1 & 19 & ${ }_{2}$ & 1 & 1 & $0 \quad 0.970$ & -13.20 & 3.3 & 0.6 & 0.0 & 0. & & 0.0 & 0.0 & 0.0 & 0.0 & 0.0 & 0.0 \\
\hline 20 & Bus 2 & 20 & $\pi 2$ & 1 & 1 & $0 \quad 0.964$ & $4-13.41$ & 2.3 & 1.0 & 0.0 & 0. & & 0.0 & 0.0 & 0.0 & 0.0 & 0.0 & 0.0 \\
\hline 21 & Bus 2 & 21 & $\pi 3$ & 1 & 1 & 01.008 & -12.89 & 0.0 & 0.0 & 0.0 & 0. & & 0.0 & 0.0 & 0.0 & 0.0 & 0.0 & 0.0 \\
\hline 22 & Bus 2 & 22 & $\pi 3$ & 1 & 1 & 01.010 & -12.84 & 0.0 & 0.0 & 0.0 & 0. & & 0.0 & 0.0 & 0.0 & 0.0 & 0.0 & 0.0 \\
\hline 23 & Bus 2 & 23 & $\pi 3$ & 1 & 1 & 01.008 & -12.91 & 6.3 & 2.1 & 0.0 & 0. & & 0.0 & 0.0 & 0.0 & 0.0 & 0.0 & 0.0 \\
\hline 24 & Bus 2 & 24 & w3 & 1 & 1 & a 0.999 & -13.25 & 0.0 & 0.0 & 0.0 & a. & & 0.0 & 0.0 & 0.0 & 0.0 & 0.0 & 0.0 \\
\hline 25 & Bus 2 & 2.5 & ॠ4 & 1 & 1 & $0 \quad 0.982$ & -18.13 & 6.3 & 3.2 & 0.0 & a. & & 0.0 & 0.0 & 0.0 & 0.0 & 0.0 & 0.0 .59 \\
\hline 26 & Bus 2 & 26 & \$5 & 1 & 1 & $0 \quad 0.959$ & -12.95 & 0.0 & 0.0 & 0.0 & a. & & 0.0 & 0.0 & 0.0 & 0.0 & 0.0 & 0.0 \\
\hline 27 & Bus 2 & 27 & $\$ 5$ & 1 & 1 & $0 \quad 0.982$ & -11.48 & 9.3 & 0.5 & 0.0 & a. & & 0.0 & 0.0 & 0.0 & 0.0 & 0.0 & 0.0 \\
\hline 28 & Bus 2 & 28 & v5 & 1 & 1 & $0 \quad 0.997$ & -10.45 & 4.6 & 2.3 & 0.0 & 0 & & 0.0 & 0.0 & 0.0 & 0.0 & 0.0 & 0.0 \\
\hline 29 & Bus 2 & 29 & $\mathrm{\psi 5}$ & 1 & 1 & 01.010 & -9.75 & 17.0 & 2.6 & 0.0 & 0 & & 0.0 & 0.0 & 0.0 & 0.0 & 0.0 & 0.0 \\
\hline 30 & Bus 3 & 30 & ఛ4 & 1 & 1 & 00.962 & -18.68 & 3.6 & 1.8 & 0.0 & 0 & & 0.0 & 0.0 & 0.0 & 0.0 & 0.0 & 0.0 \\
\hline 31 & Bus 3 & 31 & W4 & 1 & 1 & $0 \quad 0.936$ & -19.34 & 5.8 & 2.9 & 0.0 & 0 & & 0.0 & 0.0 & 0.0 & 0.0 & 0.0 & 0.0 \\
\hline 32 & Bus 3 & 32 & ॠ4 & 1 & 1 & $0 \quad 0.949$ & -18.46 & 1.6 & 0.0 & 0.0 & 0. & & 0.0 & 0.0 & 0.0 & 0.0 & 0.0 & 0.0 \\
\hline 33 & Bus 3 & 33 & $\pi 4$ & 1 & 1 & 00.947 & -18.50 & 3.8 & 1.9 & 0.0 & 0. & & 0.0 & 0.0 & 0.0 & 0.0 & 0.0 & 0.0 \\
\hline 34 & Eus 3 & 34 & 13 & 1 & 1 & 00.959 & -14.10 & 0.0 & 0.0 & 0.0 & 0 & & 0.0 & 0.0 & 0.0 & 0.0 & 0.0 & 0.0 \\
\hline 35 & Bus 3 & 35 & w3 & 1 & 1 & 00.966 & -13.86 & 6.0 & 3.0 & 0.0 & 0. & & 0.0 & 0.0 & 0.0 & 0.0 & 0.0 & 0.0 \\
\hline 36 & Eus 3 & 36 & w3 & 1 & 1 & 00.976 & -13.59 & 0.0 & 0.0 & 0.0 & 0 & & 0.0 & 0.0 & 0.0 & 0.0 & 0.0 & 0.0 \\
\hline 37 & Eus 3 & 37 & $\pi 3$ & 1 & 1 & 00.985 & -13.41 & 0.0 & 0.0 & 0.0 & 0 & & 0.0 & 0.0 & 0.0 & 0.0 & 0.0 & 0.0 \\
\hline 38 & Bus 3 & 38 & $\pi 3$ & 1 & 1 & 01.013 & -12.71 & 14.0 & 7.0 & 0.0 & 0 & & 0.0 & 0.0 & 0.0 & 0.0 & 0.0 & 0.0 \\
\hline 39 & Eus 3 & 39 & w3 & 1 & 1 & 00.983 & -13.46 & 0.0 & 0.0 & 0.0 & 0. & & 0.0 & 0.0 & 0.0 & 0.0 & 0.0 & 0.0 \\
\hline 40 & Fus 4 & 40 & v3 & 1 & 1 & 00.973 & -13.62 & 0.0 & 0.0 & 0.0 & 0. & & 0.0 & 0.0 & 0.0 & 0.0 & 0.00 & 0.0 \\
\hline 41 & Tazeu & mell & Ve & 1 & 1 & 00.996 & -14.05 & 6.3 & 3.0 & 0.0 & 0. & & 0.0 & 0.0 & 0.0 & 0.0 & 0.0 & 0.0 \\
\hline 42 & Bus 4 & 42 & Ve & 1 & 1 & 00.966 & -15.50 & 7.1 & 4.4 & 0.0 & 0. & & 0.0 & 0.0 & 0.0 & 0.0 & 0.0 & 0.0 \\
\hline 43 & Tazеш & tirell & v7 & 1 & 1 & 01.010 & -11.33 & 2.0 & 1.0 & 0.0 & 0. & & 0.0 & 0.0 & 0.0 & 0.0 & 0.0 & 0.0 \\
\hline 44 & Buz 4 & 44 & v3 & 1 & 1 & 01.017 & -11.86 & 12.0 & 1.8 & 0.0 & 0. & & 0.0 & 0.0 & 0.0 & 0.0 & 0.0 & 0.0 \\
\hline 45 & Bus 4 & 45 & v3 & 1 & 1 & 01.036 & -9.25 & 0.0 & 0.0 & 0.0 & 0. & & 0.0 & 0.0 & 0.0 & 0.0 & 0.0 & 0.0 \\
\hline 46 & Fus 4 & 46 & $v^{3}$ & 1 & 1 & 01.050 & -11.69 & 0.0 & 0.0 & 0.0 & 0. & & 0.0 & 0.0 & 0.0 & 0.0 & 0.0 & 0.0 \\
\hline 47 & Fus 4 & 47 & $v_{3}$ & 1 & 1 & 01.033 & -12.49 & 29.7 & 11.6 & 0.0 & 0. & & 0.0 & 0.0 & 0.0 & 0.0 & 0.0 & 0.0 \\
\hline 48 & Eus 4 & 48 & v3 & 1 & 1 & 01.027 & -12.59 & 0.0 & 0.0 & 0.0 & 0. & & 0.0 & 0.0 & 0.0 & 0.0 & 0.0 & 0.0 \\
\hline 49 & Eus 4 & 49 & v3 & 1 & 1 & 01.036 & -12.92 & 18.0 & 8.5 & 0.0 & 0. & & 0.0 & 0.0 & 0.0 & 0.0 & 0.0 & 0.0 \\
\hline 50 & Eus 5 & 50 & v3 & 1 & 1 & 01.023 & -13.39 & 21.0 & 10.5 & 0.0 & 0. & & 0.0 & 0.0 & 0.0 & 0.0 & 0.0 & 0.0 \\
\hline 51 & Eus 5 & 51 & v3 & 1 & 1 & $\begin{array}{ll}01.052 \\
\end{array}$ & -12.52 & 18.0 & 5.3 & 0.0 & 0. & & 0.0 & 0.0 & 0.0 & 0.0 & 0.0 & 0.0 \\
\hline 52 & Bus 5 & & V.5 & 1 & 1 & 00.980 & -11.47 & 4.9 & 2.2 & 0.0 & 0. & & 0.0 & 0.0 & 0.0 & 0.0 & 0.0 & 0.0 \\
\hline 53 & Bus 5 & 53 & V5 & 1 & 1 & 00.971 & -12.23 & 20.0 & 10.0 & 0.0 & 0. & & 0.0 & 0.0 & 0.0 & 0.0 & 0.0 & 0.063 \\
\hline 54 & Bus 5 & 54 & V.5 & 1 & 1 & 00.996 & -11.69 & 4.1 & 1.4 & 0.0 & 0. & & 0.0 & 0.0 & 0.0 & 0.0 & 0.0 & 0.0 \\
\hline 55 & $\operatorname{salto}$ & wille & e $\mathrm{v} 5$ & 1 & 1 & 01.031 & -10.70 & 6.6 & 3.4 & 0.0 & 0. & & 0.0 & 0.0 & 0.0 & 0.0 & 0.0 & 0.0 \\
\hline 56 & Fus 5 & & v6 & 1 & 1 & 00.966 & -16.04 & 7.6 & 2.2 & 0.0 & 0. & & 0.0 & 0.0 & 0.0 & 0.0 & 0.0 & 0.0 \\
\hline 57 & Eus 5 & 57 & VE & 1 & 1 & 00.965 & -16.56 & 6.7 & 2.0 & 0.0 & 0. & & 0.0 & 0.0 & 0.0 & 0.0 & 0.0 & 0.0 \\
\hline-999 & & & & & & & & & & & & & & & & & & \\
\hline BRAND & $\mathrm{H}$ DAT & $\mathrm{ATA}$ & FOLLOD & & & & & 80 & $\mathrm{H}$ & & & & & & & & & \\
\hline 1 & 2 & 21 & 11 & 0 & 0.0 & 0083 & 0.0280 & 0.1290 & a & 0 & 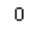 & 00 & 0.0 & & 0.00 .0 & 0.0 & 0.0 & 0.0 \\
\hline 2 & 3 & 81 & 11 & 0 & 0.0 & 0298 & 0.0850 & 0.0818 & 0 & 0 & 0 & 00 & 0.0 & & 0.00 .0 & 0.0 & 0.0 & 0.0 \\
\hline 3 & 4 & 41 & 11 & 0 & 0.0 & 112 & 0.0366 & 0.0580 & 0 & 0 & 0 & 00 & 0.0 & & 0.00 .0 & 0.0 & 0.0 & 0.0 \\
\hline 4 & 5 & 51 & 11 & 0 & 0.0 & 0625 & 0.1320 & 0.0258 & 0 & 0 & 0 & 00 & 0.0 & & 0.00 .0 & 0.0 & 0.0 & 0.0 \\
\hline 4 & 6 & 51 & 11 & 0 & 0.0 & 0430 & 0.1480 & 0.0348 & a & 0 & 0 & 00 & 0.0 & & 0.00 .0 & 0.0 & 0.0 & 0.0 \\
\hline 6 & 7 & 1 & 11 & 0 & 0.0 & 9200 & 0.1020 & 0.0276 & 0 & 0 & 0 & 00 & 0.0 & & 0.00 .0 & 0.0 & 0.0 & 0.0 \\
\hline 6 & 8 & 81 & 11 & 0 & 0.0 & 9339 & 0.1730 & 0.0470 & 0 & 0 & 0 & 00 & 0.0 & & 0.00 .0 & 0.0 & 0.0 & 0.0 \\
\hline 8 & 9 & 1 & 11 & 0 & 0.0 & 0099 & 0.0505 & 0.0548 & 0 & 0 & 0 & 00 & 0.0 & & 0.00 .0 & 0.0 & 0.0 & 0.0 \\
\hline 9 & 10 & 1 & 11 & 0 & 0.0 & 0.369 & 0.1679 & 0.0440 & a & 0 & 0 & 00 & 0.0 & & 0.00 .0 & 0.0 & 0.0 & 0.0 \\
\hline 9 & 11 & 1 & 11 & 0 & 0.0 & 0258 & 0.0848 & 0.0218 & 0 & 0 & 0 & 00 & 0.0 & & 0.00 .0 & 0.0 & 0.0 & 0.0 \\
\hline 9 & 12 & 21 & 11 & 0 & 0.0 & 0648 & 0.2950 & 0.0772 & 0 & 0 & 0 & 00 & 0.0 & & 0.00 .0 & 0.0 & 0.0 & 0.0 \\
\hline 9 & 13 & 1 & 11 & 0 & 0.0 & 1481 & 0.1580 & 0.0406 & 0 & 0 & 0 & 00 & 0.0 & & 0.00 .0 & 0.0 & 0.0 & 0.0 \\
\hline 13 & 14 & $\begin{array}{ll}71 \\
1\end{array}$ & 11 & 0 & 0.0 & 1132 & 0.0434 & 0.0110 & a & 0 & 0 & 00 & 0.0 & & 0.00 .0 & 0.0 & 0.0 & 0.0 \\
\hline 13 & 1.5 & 51 & 11 & 0 & 0.0 & 0269 & 0.0869 & 0.0230 & 0 & 0 & 0 & 00 & 0.0 & & 0.00 .0 & 0.0 & 0.0 & 0.0 \\
\hline 1 & 1.5 & 51 & 11 & 0 & 0.0 & 178 & 0.0910 & 0.0988 & 0 & 0 & 0 & 00 & 0.0 & & 0.00 .0 & 0.0 & 0.0 & 0.0 \\
\hline 1 & 16 & 51 & 11 & 0 & 0.0 & 9454 & 0.2060 & 0.0546 & 0 & 0 & 0 & 00 & 0.0 & & 0.00 .0 & 0.0 & 0.0 & 0.0 \\
\hline 1 & 17 & 1 & 11 & 0 & 0.0 & 0238 & 0.1080 & 0.0286 & a & 0 & 0 & 00 & 0.0 & & 0.00 .0 & 0.0 & 0.0 & 0.0 \\
\hline 3 & 1.5 & 51 & 11 & 0 & 0.0 & 162 & 0.0530 & 0.0544 & 0 & 0 & 0 & 00 & 0.0 & & 0.00 .0 & 0.0 & 0.0 & 0.0 \\
\hline 4 & 18 & 81 & 11 & 1 & 0.0 & & 0.5550 & 0.0 & 0 & 0 & 0 & 00 & 0.9 & & 0.00 .0 & 0.0 & 0.0 & 0.0 \\
\hline 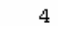 & 18 & 81 & 11 & 1 & 0.0 & & 0.4300 & 0.0 & 0 & 0 & 0 & 00 & 0.9 & & 0.00 .0 & 0.0 & 0.0 & 0.0 \\
\hline
\end{tabular}




\begin{tabular}{|c|c|c|c|c|c|c|c|c|c|c|c|c|c|c|c|c|}
\hline 5 & 6 & 1 & 11 & $0 \quad 0.0302$ & 0.0641 & 0.0124 & 0 & 0 & 0 & 0 & 0.0 & 0.00 .0 & 0.0 & 0.0 & 0.0 & 0.0 \\
\hline 7 & 8 & 1 & 11 & $0 \quad 0.0139$ & 0.0712 & 0.0194 & 0 & 0 & 0 & 0 & 0.0 & 0.00 .0 & 0.0 & 0.0 & 0.0 & 0.0 \\
\hline 10 & 12 & 1 & 11 & $0 \quad 0.0277$ & 0.1262 & 0.0328 & 0 & 0 & 0 & 0 & 0.0 & 0.00 .0 & 0.0 & 0.0 & 0.0 & 0.0 \\
\hline 11 & 13 & 1 & 11 & $0 \quad 0.0223$ & 0.0732 & 0.0188 & 0 & 0 & 0 & 0 & 0.0 & 0.00 .0 & 0.0 & 0.0 & 0.0 & 0.0 \\
\hline 12 & 13 & 1 & 11 & $0 \quad 0.0178$ & 0.0 .580 & 0.0604 & 0 & 0 & 0 & 0 & 0.0 & 0.00 .0 & 0.0 & 0.0 & 0.0 & 0.0 \\
\hline 12 & 16 & 1 & 11 & $0 \quad 0.0180$ & 0.0813 & 0.0216 & 0 & 0 & 0 & 0 & 0.0 & 0.00 .0 & 0.0 & 0.0 & 0.0 & 0.0 \\
\hline 12 & 17 & 1 & 11 & $0 \quad 0.0397$ & 0.1790 & 0.0476 & 0 & 0 & 0 & 0 & 0.0 & 0.00 .0 & 0.0 & 0.0 & 0.0 & 0.0 \\
\hline 14 & 1.5 & 1 & 11 & $0 \quad 0.0171$ & 0.0 .547 & 0.0148 & 0 & 0 & 0 & 0 & 0.0 & 0.00 .0 & 0.0 & 0.0 & 0.0 & 0.0 \\
\hline 18 & 19 & 1 & 11 & $0 \quad 0.4610$ & 0.6850 & 0.0 & 0 & 0 & 0 & 0 & 0.0 & 0.00 .0 & 0.0 & 0.0 & 0.0 & 0.0 \\
\hline 19 & 20 & 1 & 11 & $0 \quad 0.2830$ & 0.4340 & 0.0 & 0 & 0 & 0 & 0 & 0.0 & 0.00 .0 & 0.0 & 0.0 & 0.0 & 0.0 \\
\hline 21 & 20 & 1 & 11 & 10.0 & 0.7767 & 0.0 & 0 & 0 & 0 & 0 & 1.043 & 0.00 .0 & 0.0 & 0.0 & 0.0 & 0.0 \\
\hline 21 & 22 & 1 & 11 & $0 \quad 0.0736$ & 0.1170 & 0.0 & 0 & 0 & 0 & 0 & 0.0 & 0.00 .0 & 0.0 & 0.0 & 0.0 & 0.0 \\
\hline 22 & 23 & 1 & 11 & $0 \quad 0.0099$ & 0.0152 & 0.0 & 0 & 0 & 0 & 0 & 0.0 & 0.00 .0 & 0.0 & 0.0 & 0.0 & 0.0 \\
\hline 23 & 24 & 1 & 11 & $0 \quad 0.1660$ & 0.2560 & 0.0084 & 0 & 0 & 0 & 0 & 0.0 & 0.00 .0 & 0.0 & 0.0 & 0.0 & 0.0 \\
\hline 24 & 25 & 1 & 11 & 10.0 & 1.1820 & 0.0 & 0 & 0 & 0 & 0 & 1.000 & 0.00 .0 & 0.0 & 0.0 & 0.0 & 0.0 \\
\hline 24 & 25 & 1 & 11 & $0 \quad 0.0$ & 1.2300 & 0.0 & 0 & 0 & 0 & 0 & 1.000 & 0.00 .0 & 0.0 & 0.0 & 0.0 & 0.0 \\
\hline 24 & 26 & 1 & 11 & 10.0 & 0.0473 & 0.0 & 0 & 0 & 0 & 0 & 1.043 & 0.00 .0 & 0.0 & 0.0 & 0.0 & 0.0 \\
\hline 26 & 27 & 1 & 11 & $0 \quad 0.16 .50$ & 0.2540 & 0.0 & 0 & 0 & 0 & 0 & 0.0 & 0.00 .0 & 0.0 & 0.0 & 0.0 & 0.0 \\
\hline 27 & 28 & 1 & 11 & $0 \quad 0.0618$ & 0.09 .54 & 0.0 & 0 & 0 & 0 & 0 & 0.0 & 0.00 .0 & 0.0 & 0.0 & 0.0 & 0.0 \\
\hline 28 & 29 & 1 & 11 & $0 \quad 0.0418$ & 0.0 .587 & 0.0 & 0 & 0 & 0 & 0 & 0.0 & 0.00 .0 & 0.0 & 0.0 & 0.0 & 0.0 \\
\hline 7 & 29 & 1 & 11 & 10.0 & 0.0648 & 0.0 & 0 & 0 & 0 & 00 & 0.967 & 0.00 .0 & 0.0 & 0.0 & 0.0 & 0.0 \\
\hline 25 & 30 & 1 & 11 & $0 \quad 0.1350$ & 0.2020 & 0.0 & 0 & 0 & 0 & 0 & 0.0 & 0.00 .0 & 0.0 & 0.0 & 0.0 & 0.0 \\
\hline 30 & 31 & 1 & 11 & $0 \quad 0.3260$ & 0.4970 & 0.0 & 0 & 0 & 0 & 0 & 0.0 & 0.00 .0 & 0.0 & 0.0 & 0.0 & 0.0 \\
\hline 31 & 32 & 1 & 11 & $0 \quad 0.5070$ & 0.75 .50 & 0.0 & 0 & 0 & 0 & 0 & 0.0 & 0.00 .0 & 0.0 & 0.0 & 0.0 & 0.0 \\
\hline 32 & 33 & 1 & 11 & $0 \quad 0.0392$ & 0.0360 & 0.0 & 0 & 0 & 0 & 0 & 0.0 & 0.00 .0 & 0.0 & 0.0 & 0.0 & 0.0 \\
\hline 34 & 32 & 1 & 11 & 10.0 & 0.9530 & 0.0 & 0 & 0 & 0 & 0 & 0.975 & 0.00 .0 & 0.0 & 0.0 & 0.0 & 0.0 \\
\hline 34 & 35 & 1 & 11 & $0 \quad 0.0520$ & 0.0780 & 0.0032 & 0 & 0 & 0 & 0 & 0.0 & 0.00 .0 & 0.0 & 0.0 & 0.0 & 0.0 \\
\hline 35 & 36 & 1 & 11 & $\begin{array}{ll}0 & 0.0430\end{array}$ & 0.0537 & 0.0016 & 0 & 0 & 0 & 0 & 0.0 & 0.00 .0 & 0.0 & 0.0 & 0.0 & 0.0 \\
\hline 36 & 37 & 1 & 11 & $0 \quad 0.0290$ & 0.0366 & 0.0 & 0 & 0 & 0 & 0 & 0.0 & 0.00 .0 & 0.0 & 0.0 & 0.0 & 0.0 \\
\hline 37 & 30 & 1 & 11 & $0 \quad 0.0651$ & 0.1009 & 0.0020 & 0 & 0 & 0 & 0 & 0.0 & 0.00 .0 & 0.0 & 0.0 & 0.0 & 0.0 \\
\hline 37 & 39 & 1 & 11 & $0 \quad 0.0239$ & 0.0379 & 0.0 & 0 & 0 & 0 & 0 & 0.0 & 0.00 .0 & 0.0 & 0.0 & 0.0 & 0.0 \\
\hline 36 & 40 & 1 & 11 & $0 \quad 0.0300$ & 0.0466 & 0.0 & 0 & 0 & 0 & 0 & 0.0 & 0.00 .0 & 0.0 & 0.0 & 0.0 & 0.0 \\
\hline 22 & 38 & 1 & 11 & $0 \quad 0.0192$ & 0.0295 & 0.0 & 0 & 0 & 0 & 0 & 0.0 & 0.00 .0 & 0.0 & 0.0 & 0.0 & 0.0 \\
\hline 11 & 41 & 1 & 11 & 10.0 & 0.7490 & 0.0 & 0 & 0 & 0 & 0 & 0.955 & 0.00 .0 & 0.0 & 0.0 & 0.0 & 0.0 \\
\hline 41 & 42 & 1 & 11 & $0 \quad 0.2070$ & 0.3520 & 0.0 & 0 & 0 & 0 & 0 & 0.0 & 0.00 .0 & 0.0 & 0.0 & 0.0 & 0.0 \\
\hline 41 & 43 & 1 & 11 & $0 \quad 0.0$ & 0.4120 & 0.0 & 0 & 0 & 0 & 00 & 0.0 & 0.00 .0 & 0.0 & 0.0 & 0.0 & 0.0 \\
\hline 38 & 44 & 1 & 11 & $0 \quad 0.0289$ & 0.0 .585 & 0.0020 & 0 & 0 & 0 & 0 & 0.0 & 0.00 .0 & 0.0 & 0.0 & 0.0 & 0.0 \\
\hline 15 & 45 & 1 & 11 & 10.0 & 0.1042 & 0.0 & 0 & 0 & 0 & 0 & 0.955 & 0.00 .0 & 0.0 & 0.0 & 0.0 & 0.0 \\
\hline 14 & 46 & 1 & 11 & 10.0 & 0.0735 & 0.0 & 0 & 0 & 0 & 0 & 0.900 & 0.00 .0 & 0.0 & 0.0 & 0.0 & 0.0 \\
\hline 46 & 47 & 1 & 11 & $0 \quad 0.0230$ & 0.0680 & 0.0032 & 0 & 0 & 0 & 0 & 0.0 & 0.00 .0 & 0.0 & 0.0 & 0.0 & 0.0 \\
\hline 47 & 48 & 1 & 11 & $\begin{array}{ll}0 & 0.0182\end{array}$ & 0.0233 & 0.0 & 0 & 0 & 0 & 0 & 0.0 & 0.00 .0 & 0.0 & 0.0 & 0.0 & 0.0 \\
\hline 48 & 49 & 1 & 11 & $0 \quad 0.0834$ & 0.1290 & 0.0048 & 0 & 0 & 0 & 0 & 0.0 & 0.00 .0 & 0.0 & 0.0 & 0.0 & 0.0 \\
\hline 49 & 50 & 1 & 11 & $0 \quad 0.0801$ & 0.1280 & 0.0 & 0 & 0 & 0 & 0 & 0.0 & 0.00 .0 & 0.0 & 0.0 & 0.0 & 0.0 \\
\hline 50 & 51 & 1 & 11 & $0 \quad 0.1386$ & 0.2200 & 0.0 & 0 & 0 & 0 & 00 & 0.0 & 0.00 .0 & 0.0 & 0.0 & 0.0 & 0.0 \\
\hline 10 & 51 & 1 & 11 & 10.0 & 0.0712 & 0.0 & 0 & 0 & 0 & 0 & 0.930 & 0.00 .0 & 0.0 & 0.0 & 0.0 & 0.0 \\
\hline 13 & 49 & 1 & 11 & $1 \quad 0.0$ & 0.1910 & 0.0 & 0 & 0 & 0 & 0 & 0.895 & 0.00 .0 & 0.0 & 0.0 & 0.0 & 0.0 \\
\hline 29 & 52 & 1 & 11 & $\begin{array}{ll}0 & 0.1442\end{array}$ & 0.1870 & 0.0 & 0 & 0 & 0 & 0 & 0.0 & 0.00 .0 & 0.0 & 0.0 & 0.0 & 0.0 \\
\hline 52 & 53 & 1 & 11 & $0 \quad 0.0762$ & 0.0984 & 0.0 & 0 & 0 & 0 & 00 & 0.0 & 0.00 .0 & 0.0 & 0.0 & 0.0 & 0.0 \\
\hline 53 & 54 & 1 & 11 & $0 \quad 0.1878$ & 0.2320 & 0.0 & 0 & 0 & 0 & 0 & 0.0 & 0.00 .0 & 0.0 & 0.0 & 0.0 & 0.0 \\
\hline 54 & 55 & 1 & 11 & $0 \quad 0.1732$ & 0.2265 & 0.0 & 0 & 0 & 0 & 0 & 0.0 & 0.00 .0 & 0.0 & 0.0 & 0.0 & 0.0 \\
\hline 11 & 43 & 1 & 11 & 10.0 & 0.1530 & 0.0 & 0 & 0 & 0 & 0 & 0.958 & 0.00 .0 & 0.0 & 0.0 & 0.0 & 0.0 \\
\hline 44 & 45 & 1 & 11 & $0 \quad 0.0624$ & 0.1242 & 0.0040 & 0 & 0 & 0 & $0 \mathrm{c}$ & 0.0 & 0.00 .0 & 0.0 & 0.0 & 0.0 & 0.0 \\
\hline 40 & 56 & 1 & 11 & 10.0 & 1.1950 & 0.0 & 0 & 0 & 0 & 00 & 0.958 & 0.00 .0 & 0.0 & 0.0 & 0.0 & 0.0 \\
\hline 56 & 41 & 1 & 11 & $0 \quad 0.5530$ & 0.5490 & 0.0 & 0 & 0 & 0 & 00 & 0.0 & 0.00 .0 & 0.0 & 0.0 & 0.0 & 0.0 \\
\hline 56 & 42 & 1 & 11 & $0 \quad 0.2125$ & 0.3540 & 0.0 & 0 & 0 & 0 & 00 & 0.0 & 0.00 .0 & 0.0 & 0.0 & 0.0 & 0.0 \\
\hline 39 & 57 & 1 & 11 & 10.0 & 1.3550 & 0.0 & 0 & 0 & 0 & 00 & 0.980 & 0.00 .0 & 0.0 & 0.0 & 0.0 & 0.0 \\
\hline 57 & 56 & 1 & 11 & $0 \quad 0.1740$ & 0.2600 & 0.0 & 0 & 0 & 0 & 00 & 0.0 & 0.00 .0 & 0.0 & 0.0 & 0.0 & 0.0 \\
\hline 38 & 49 & 1 & 11 & $\begin{array}{ll}0 & 0.1150\end{array}$ & 0.1770 & 0.0060 & 0 & 0 & 0 & 00 & 0.0 & 0.00 .0 & 0.0 & 0.0 & 0.0 & 0.0 \\
\hline 38 & 48 & 1 & 11 & $0 \quad 0.0312$ & 0.0482 & 0.0 & 0 & 0 & 0 & 00 & 0.0 & 0.00 .0 & 0.0 & 0.0 & 0.0 & 0.0 \\
\hline 9 & 55 & 1 & 11 & 10.0 & 0.1205 & 0.0 & 0 & 0 & 0 & $0 \mathrm{c}$ & 0.940 & 0.00 .0 & 0.0 & 0.0 & 0.0 & 0.0 \\
\hline
\end{tabular}


- Banco de dados do sistema IEEE - 118 barras

BUS DATA FOLLOTIS

1. Fiversale V2 $11020.055 \quad 10.07$ 2 Pokagon V2 1 1 $100.971 \quad 11.22$

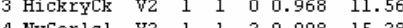
$\begin{array}{lllllll}4 \text { NoCarlsl } & \text { V2 } & 1 & 1 & 2 & 0.998 & 15.28 \\ 5.01 i v e & \text { V2 } & 1 & 1 & 0 & 1.002 & 15.73\end{array}$ $\begin{array}{lllllll}5 \text { Olive } & \text { V2 } & 1 & 1 & 0 & 1.002 & 15.73 \\ 6 \text { Kankakee } & \text { V2 } & 1 & 1 & 2 & 0.990 & 13.00\end{array}$

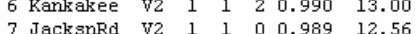

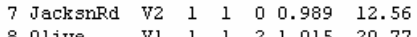
8 Olive V1 $110101.015 \quad 20.77$

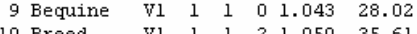
10 Ereed V1 $1101201.050 \quad 35.61$

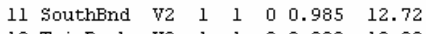

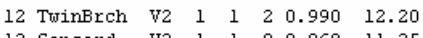
13 Concord V2 $110100.960 \quad 11.35$ 14 Goshendt V2 1 l 00.984 11.50

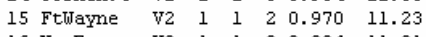

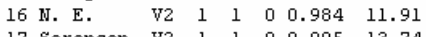
18 McKinley V2 11 l $20.973 \quad 11.53$ 19 Lincoln v2 1 l $230.963 \quad 11.05$

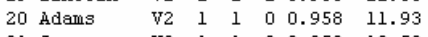

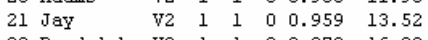
22 Randolph V2 1 l 00.97016 .08

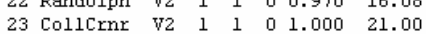

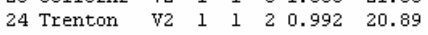

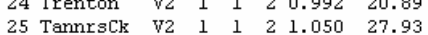

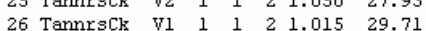

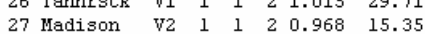
$\begin{array}{lllllll}27 \text { Madison } & \text { V2 } & 1 & 1 & 2 & 0.968 & 15.35 \\ 28 \text { Mullin } & \text { V2 } & 1 & 1 & 0 & 0.962 & 13.62\end{array}$ $\begin{array}{lllllll}28 \text { Mullin } & \text { V2 } & 1 & 1 & 0 & 0.962 & 13.62 \\ 29 \text { Grant } & \text { V2 } & 1 & 1 & 0 & 0.963 & 12.63\end{array}$ 30 Sorenson V1 1 l 1100.968 (8.79

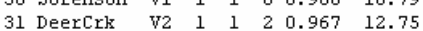

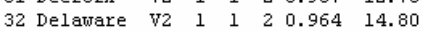

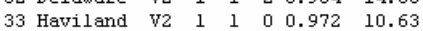

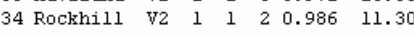
35 Westima V2 1 l $00.961 \quad 10.87$ $\begin{array}{lllllll}36 & \text { sterling V2 } & 1 & 1 & 2 & 0.980 & 10.87\end{array}$ 37 Eastilina V2 1 l $100.992 \quad 11.77$ $\begin{array}{lllllll}36 \text { Eastifina V1 } & 1 & 1 & 0 & 0.962 & 16.91\end{array}$ $\begin{array}{lllllll}39 \text { NuLibrty } & \text { V2 } & 1 & 1 & 0 & 0.970 & 8.41 \\ \end{array}$

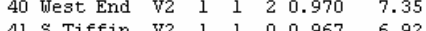

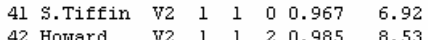

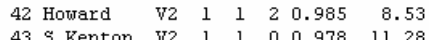
43 s. Kentan V2 11 1 $00.978 \quad 11.28$ 44 WWVernon V2 110100.96513 .82

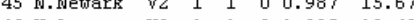

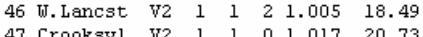
$\begin{array}{llllllll}47 \text { Crooksvl V2 } & 1 & 1 & 0 & 1.017 & 20.73\end{array}$ $\begin{array}{lllllllll}48 & \text { Zanesvll } & \text { V2 } & 1 & 1 & 0 & 1.021 & 19.93\end{array}$

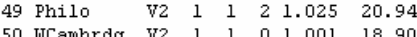
$\begin{array}{lllllll}51 \text { Newcmrst V2 } & 1 & 1 & 0 & 0.967 & 16.28\end{array}$ 52 scoshoet v2 1 1 $100.957 \quad 15.32$

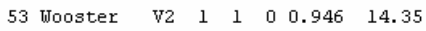
$\begin{array}{lllllll}54 & \text { Torrey V2 } & 1 & 1 & 2 & 0.955 & 15.26\end{array}$

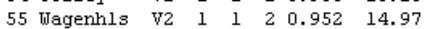

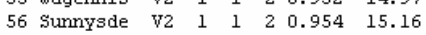
57 WwPhill V2 1 l $100.971 \quad 16.36$ 58 WNuPhil2 V2 1 l 0.0 .959 15.51 $\begin{array}{lllllll}58 \text { WWTh112 } & \text { V2 } & 1 & 1 & 0 & 0.959 & 15.51 \\ 59 \text { Tidd } & \text { V2 } & 1 & 1 & 2 & 0.985 & 19.37\end{array}$

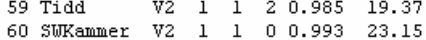

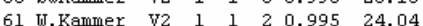
62 Natrium V2 $1111020.996 \quad 23.43$ 63 Tidd V1 1 l 00.969 22.75 64 Kamier V1 $110100.984 \quad 24.52$

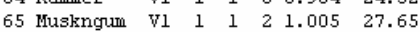

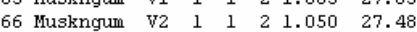
67 suminerf

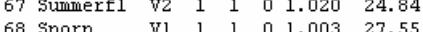

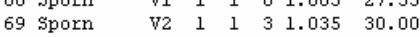

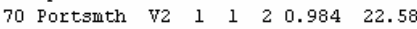
71 WPartsint V2 110100.98722 .15 72 Hillsbro V2 11 l $220.980 \quad 20.98$ 73 Sargents V2 11 1 $220.991 \quad 21.94$ 74 Bellefnt v2 $110120.958 \quad 21.64$ $\begin{array}{llllllll}75 & \text { sthPoint v2 } & 1 & 1 & 0 & 0.967 & 22.91\end{array}$

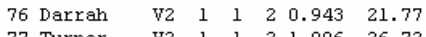

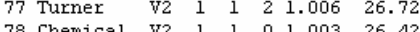
78 Chemical V2 110100030.42

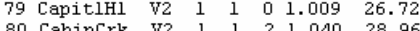
$\begin{array}{lllllll}80 \text { CabinCrk } & \text { V2 } & 1 & 1 & 2 & 1.040 & 28.96 \\ 81 \text { Kanatiha } & \text { V1 } & 1 & 1 & 0 & 0.997 & 28.10\end{array}$ 62 Logan VZ 11 l 100.969 27.24 83 sprigg V2 $11100.985 \quad 28.42$

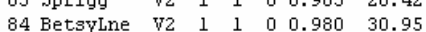

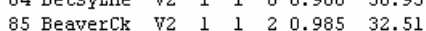

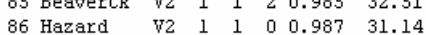
86 Hazard V2 $110100.987 \quad 31.14$

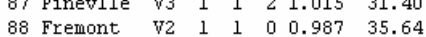
$\begin{array}{llllllll}88 & \text { Fremont } & \text { WZ } & 1 & 1 & 0 & 0.987 & 35.64 \\ 89 & \text { ClinchRo } & \text { V2 } & 1 & 1 & 2 & 1.005 & 39.69\end{array}$ $\begin{array}{lllllll}90 \text { Holston } & \text { W2 } & 1 & 1 & 2 & 0.985 & 33.29\end{array}$
57 ITEMS

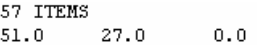

$39.0 \quad 0.0$

$30.0 \quad 12.0 \quad-9.0$

$0.0 \quad 0.0$

$19.0 \quad 2.0$

$0.0 \quad 0.0$

$0.0 \quad 0.0$

$\begin{array}{rr}0.0 \\ 70.0 & 23.0 \\ 7.0 & 10.0\end{array}$

$47.0 \quad 10.0$

$\begin{array}{rr}34.0 & 16.0 \\ 14.0 & 1.0\end{array}$

90.030

25.010 .0

11.0
60.0

18.0

14.0

10.

0.

0.0

62.0

17.0
24.0

0.0

43.0

23.1

33.0

31

$\begin{array}{rr}31.0 & 17 \\ 0.0 & 0\end{array}$

27.0

27.0
20.0
37.0

37.0

18.0

53.0

28.0
34.0

67.0

17.0

17.0

16.0

23.0
113.0
63.0

63.0
84.0
12.0

12.0
277.0

277.0
78.0

0.0

77.0

0.0
0.0
0.0

0.0

0.0
39.0
28.0
0.0

0.0
0.0
66.0
0.0

0.0

0.0
0.0
68.0

47.0

61.0
71.0

39.0
130.0
0.0

0.0
54.0

20.0

11.0

24.0

21.0
0.0

48.0
0.0

0.0
78.0
25.0

3.

0.0
5.0

3.0

$0.0 \quad-13.0$

$0.0 \quad 220.0$

13.0

0.0

23.0

9.0

26.0

17.0

0.0

11.0
23.0

10.0

23.0

22.0
10.0

0.0

30.0

8.0

8.0
5.0
11.0

32.0

22.0

18.0

3.0
3.0

113.0
3.0

0.0

14.0
0.0

0.0

0.0
18.0

7.0

0.0

20.0

0.0

0.0
27.0

11.0

36.0

32.0

0.0

27.0

10.0

15.0

10.0
0.0

10.0

42.0
0.0

0.0
0.0

0.0

0.0

0.0

0.0
0.0

0.0

0.0

0.0
0.0

0.0

0.0

0. 0

0.0

0.0

0.0

0.0

0.0
0.0
0.0

0.0

0.0
0.0

0. 0

0.0

0.0

0.0
0.0

0.0

0.0

0.0
0.0
0.0

0.0

0.0

0.0
0.0
0.0

0.0

0.0

0.0
0.0
0.0

0.0
0.0
0.0

0.0

0.0
0.0
0.0

0.0

0.0
0.0

0.0
0.0
0.0

0.0
0.0
0.0

0.0

0.0

0.0

0.0

0.0
0.0
0.0
0.0

0.0

0.0

0.0
0.0
0.0

0.0
0.0
0.0

0.0

0.0

0.0
0.0
0.0

0.0

0.0

0.0

0. 0

0.0

0. 0

0.0

0.0

0.0

0.0

0.0
0.0

$\begin{array}{rrrrrrr}0.0 & 0.955 & 15.0 & -5.0 & 0.0 & 0.0 & 0 \\ 0.0 & 0.0 & 0.0 & 0.0 & 0.0 & 0.0\end{array}$

$\begin{array}{lllllll}0.0 & 0.0 & 0.0 & 0.0 & 0.0 & 0.0 & 0 \\ 0.0 & 0.0 & 0.0 & 0.0 & 0.0 & 0.0\end{array}$

$\begin{array}{llllll}0.0 & 0.998 & 300.0 & -300.0 & 0.0 & 0.0\end{array}$

$\begin{array}{rlrrrr}0.0 & 0.0 & 0.0 & 0.0 & 0.0 & -0.40 \\ 0.0 & 0.990 & 50.0 & -13.0 & 0.0 & 0.0\end{array}$

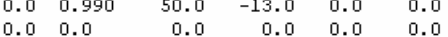

$\begin{array}{llllll}1.015 & 300.0 & -300.0 & 0.0 & 0.0\end{array}$

$\begin{array}{lllllll}0.0 & 0.0 & 0.0 & 0.0 & 0.0 & 0.0\end{array}$

$\begin{array}{llllll}0.0 & 1.050 & 200.0 & -147.0 & 0.0 & 0.0\end{array}$

$\begin{array}{rrrrrr}0.0 & 0.990 & 120.0 & -35.0 & 0.0 & 0.0\end{array}$

$\begin{array}{lllll}0.0 & 0.0 & 0.0 & 0.0 & 0.0\end{array}$

$\begin{array}{llllll}0.0 & 0.0 & 0.0 & 0.0 & 0.0 & 0.0\end{array}$

$\begin{array}{llllll}0.0 & 0.970 & 30.0 & -10.0 & 0.0 & 0.0\end{array}$

$\begin{array}{llllll}0.0 & 0.0 & 0.0 & 0.0 & 0.0 & 0.0\end{array}$

$\begin{array}{rlrrrr}0.0 & 0.0 & 0.0 & 0.0 & 0.0 & 0.0\end{array}$

$\begin{array}{llllll}0.0 & 0.962 & 24.0 & -8.0 & 0.0 & 0.0\end{array}$ 


\begin{tabular}{|c|c|c|c|c|c|c|c|}
\hline 91 & Holsto & & $\sqrt{2}$ & 1 & 1 & 20.980 & 33.31 \\
\hline 92 & Saltwl & & $v_{2}$ & 1 & 1 & 20.993 & $3 \quad 33.80$ \\
\hline 93 & Taz etre & & $v_{2}$ & 1 & 1 & 00.987 & 30.79 \\
\hline 94 & Stritch & & $v_{2}$ & 1 & 1 & 00.991 & $1 \quad 28.64$ \\
\hline 95 & Cal dwe & & $\sqrt{2}$ & 1 & 1 & 00.981 & 27.67 \\
\hline 96 & Bailey & & $\sqrt{2}$ & 1 & 1 & 00.993 & $3 \quad 27.51$ \\
\hline 97 & Sundia & & $\sqrt{2}$ & 1 & 1 & 01.011 & $1 \quad 27.88$ \\
\hline 98 & Bradle & & $\sqrt{2}$ & 1 & 1 & 01.024 & $4 \quad 27.40$ \\
\hline 99 & Hintan & & $v_{2}$ & 1 & 1 & 21.010 & $\quad 27.04$ \\
\hline 100 & Glen L & & $v_{2}$ & 1 & 1 & 21.017 & 28.03 \\
\hline 101 & Ththe & & $v_{2}$ & 1 & 1 & 00.993 & 329.61 \\
\hline 102 & Silythe & & $v_{2}$ & 1 & 1 & 00.991 & $1 \quad 32.30$ \\
\hline 103 & Clayto & & $\sqrt{2}$ & 1 & 1 & 21.001 & $1 \quad 24.44$ \\
\hline 104 & Hancod & & $\sqrt{2}$ & 1 & 1 & 20.971 & $1 \quad 21.69$ \\
\hline 10.5 & Roanok & & $\mathrm{V}_{2}$ & 1 & 1 & 20.965 & $5 \quad 20.57$ \\
\hline 106 & Clover & & $v_{2}$ & 1 & 1 & 00.962 & $2 \quad 20.32$ \\
\hline 107 & Reusen & & $v_{2}$ & 1 & 1 & 20.952 & $2 \quad 17.53$ \\
\hline 108 & Glaine & & $v_{2}$ & 1 & 1 & 00.967 & 19.30 \\
\hline 109 & Frandz 1 & & $v_{2}$ & 1 & 1 & 00.967 & 18.93 \\
\hline 110 & Fielda & & $\sqrt{2}$ & 1 & 1 & 20.973 & $3 \quad 18.09$ \\
\hline 111 & DanRiv & & $v_{2}$ & 1 & 1 & 20.980 & $\quad 19.74$ \\
\hline 112 & Danvil & & $v_{2}$ & 1 & 1 & 20.975 & $5 \quad 14.99$ \\
\hline 113 & Deer : & & $v_{2}$ & 1 & 1 & 20.993 & $3 \quad 13.74$ \\
\hline 114 & the dfo & & $\sqrt{2}$ & 1 & 1 & $0 \quad 0.960$ & 14.46 \\
\hline 115 & Medfor & & $v_{2}$ & 1 & 1 & 00.960 & 14.46 \\
\hline 116 & Kyger D & & $\mathrm{v} 2$ & 1 & 1 & 21.00 .5 & $5 \quad 27.12$ \\
\hline 117 & Corey & & $\sqrt{2}$ & 1 & 1 & $0 \quad 0.974$ & $4 \quad 10.67$ \\
\hline 118 & THuntn & & $\mathrm{V} 2$ & 1 & 1 & $0 \quad 0.949$ & 21.92 \\
\hline Find & $\mathrm{H}$ DAT & $\mathrm{FO}$ & OLLO & & & & \\
\hline 1 & 2 & 1 & 11 & 0 & 0.0 & 03030 & 0.09990 \\
\hline 1 & 3 & 1 & 11 & 0 & 0.0 & 01290 & 0.04240 \\
\hline 4 & 5 & 1 & 11 & 0 & 0.0 & 00176 & 0.00798 \\
\hline 3 & 5 & 1 & 11 & 0 & 0.0 & 02410 & 0.10000 \\
\hline 5 & 6 & 1 & 11 & 0 & 0.0 & 01190 & 0.05400 \\
\hline 6 & 7 & 1 & 11 & 0 & 0.0 & 00459 & 0.02080 \\
\hline 8 & 9 & 1 & 11 & 0 & 0.0 & 00244 & 0.03050 \\
\hline 8 & 5 & 1 & 11 & 1 & 0.0 & 00000 & 0.02670 \\
\hline 9 & 10 & 1 & 11 & 0 & 0.0 & 002.58 & 0.03220 \\
\hline 4 & 11 & 1 & 11 & 0 & 0.0 & 02090 & 0.06880 \\
\hline 5 & 11 & 1 & 11 & 0 & 0.0 & 02030 & 0.06820 \\
\hline 11 & 12 & 1 & 11 & 0 & 0.0 & 00595 & 0.01960 \\
\hline 2 & 12 & 1 & 11 & 0 & 0.0 & 01870 & 0.06160 \\
\hline 3 & 12 & 1 & 11 & 0 & 0.0 & 04840 & 0.16000 \\
\hline 7 & 12 & 1 & 11 & 0 & 0.0 & 00862 & 0.03400 \\
\hline 11 & 13 & 1 & 11 & 0 & 0.0 & 02225 & 0.07310 \\
\hline 12 & 14 & 1 & 11 & 0 & 0.0 & 021.50 & 0.07070 \\
\hline 13 & 1.5 & 1 & 11 & 0 & 0.0 & 97440 & 0.24440 \\
\hline 14 & 1.5 & 1 & 11 & 0 & 0.0 & 0.59 .50 & 0.19500 \\
\hline 12 & 16 & 1 & 11 & 0 & 0.0 & 02120 & 0.08340 \\
\hline 1.5 & 17 & 1 & 11 & 0 & 0.0 & 01320 & 0.04370 \\
\hline 16 & 17 & 1 & 11 & 0 & 0.0 & 94540 & 0.18010 \\
\hline 17 & 18 & 1 & 11 & 0 & 0.0 & 01230 & 0.05050 \\
\hline 18 & 19 & 1 & 11 & 0 & 0.0 & 91119 & 0.04930 \\
\hline 19 & 20 & 1 & 11 & 0 & 0.0 & 02520 & 0.11700 \\
\hline 15 & 19 & 1 & 11 & 0 & 0.0 & 01200 & 0.03940 \\
\hline 20 & 21 & 1 & 11 & 0 & 0.0 & 1830 & 0.08490 \\
\hline 21 & 22 & 1 & 11 & 0 & 0.0 & 02090 & 0.09700 \\
\hline 22 & 23 & 1 & 11 & 0 & 0.0 & 13420 & 0.15900 \\
\hline 23 & 24 & 1 & 11 & 0 & 0.0 & 01350 & 0.04920 \\
\hline 23 & 25 & 1 & 11 & 0 & 0.0 & 01560 & 0.08000 \\
\hline 26 & 25 & 1 & 11 & 1 & 0.0 & 00000 & 0.03820 \\
\hline 25 & 27 & 1 & 11 & 0 & 0.0 & 03180 & 0.16300 \\
\hline 27 & 28 & 1 & 11 & 0 & 0.0 & 01913 & 0.08550 \\
\hline 28 & 29 & 1 & 11 & 0 & 0.0 & 02370 & 0.09430 \\
\hline 30 & 17 & 1 & 11 & 1 & 0.0 & 00000 & 0.03660 \\
\hline 8 & 30 & 1 & 11 & 0 & 0.0 & 10431 & 0.05040 \\
\hline 26 & 30 & 1 & 11 & 0 & 0.0 & 00799 & 0.08600 \\
\hline 17 & 31 & 1 & 11 & & & 94740 & 0.15630 \\
\hline 29 & 31 & 1 & 11 & 0 & 0.0 & 01080 & 0.03310 \\
\hline 23 & 32 & 1 & 11 & 0 & & 03170 & 0.11530 \\
\hline 31 & 32 & 1 & 11 & 0 & 0.0 & 02980 & 0.09850 \\
\hline 27 & 32 & 1 & 11 & 0 & 0.0 & 02290 & 0.07550 \\
\hline 1.5 & 33 & 1 & 11 & 0 & 0.0 & 03800 & 0.12440 \\
\hline 19 & 34 & 1 & 11 & 0 & 0.0 & 07520 & 0.24700 \\
\hline 35 & 36 & 1 & 11 & 0 & 0.0 & 00224 & 0.01020 \\
\hline 3.5 & 37 & 1 & 11 & a & 0.0 & 01100 & 0.04970 \\
\hline 33 & 37 & 1 & 11 & 0 & 0.0 & 04150 & 0.14200 \\
\hline 34 & 36 & 1 & 11 & 0 & 0.0 & 00871 & 0.02680 \\
\hline 34 & 37 & 1 & 11 & 0 & 0.0 & 00256 & 0.00940 \\
\hline 36 & 37 & 1 & 11 & 1 & 0.0 & 00000 & 0.03750 \\
\hline 37 & 39 & 1 & 11 & 0 & 0.0 & 03210 & 0.10600 \\
\hline 37 & 40 & 1 & 11 & 0 & 0.0 & 05930 & 0.16800 \\
\hline 30 & 38 & 1 & 11 & 0 & 0.0 & 00464 & 0.05400 \\
\hline 39 & 40 & 1 & 11 & 0 & 0.0 & 01640 & 0.06050 \\
\hline 40 & 41 & 1 & 11 & 0 & 0.0 & 01450 & 0.04870 \\
\hline 40 & 42 & 1 & 11 & 0 & 0.0 & 05.5 .50 & 0.18300 \\
\hline 41 & 42 & 1 & 11 & 0 & 0.0 & 04100 & 0.13500 \\
\hline 43 & 44 & 1 & 11 & 0 & 0.0 & 06080 & 0.24540 \\
\hline 34 & 43 & 1 & 11 & 0 & 0.0 & 04130 & 0.16810 \\
\hline 44 & 45 & 1 & 11 & 0 & & 2240 & 0.09010 \\
\hline 45 & 46 & 1 & 11 & 0 & 0.0 & 04000 & 0.13560 \\
\hline
\end{tabular}




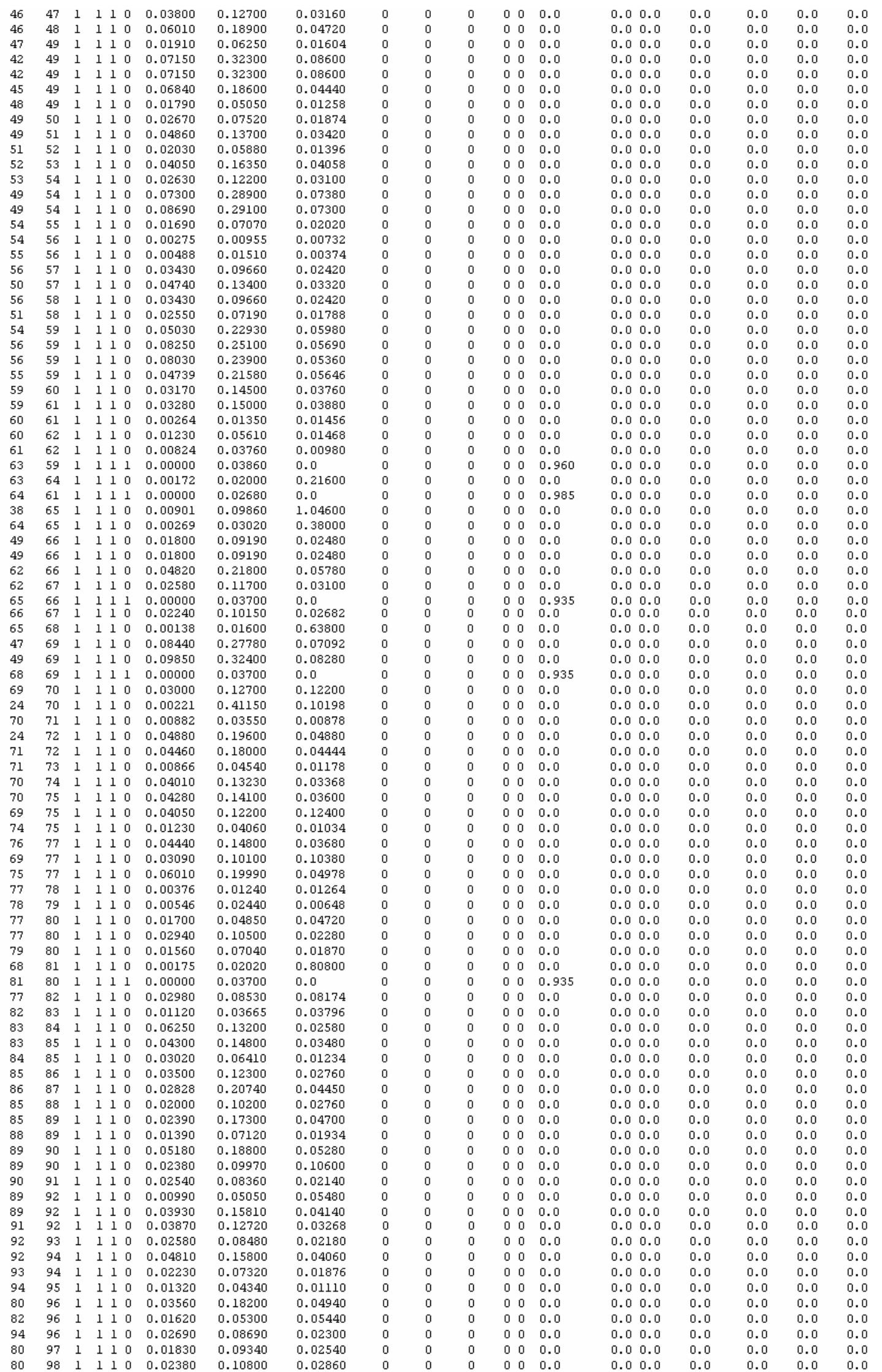




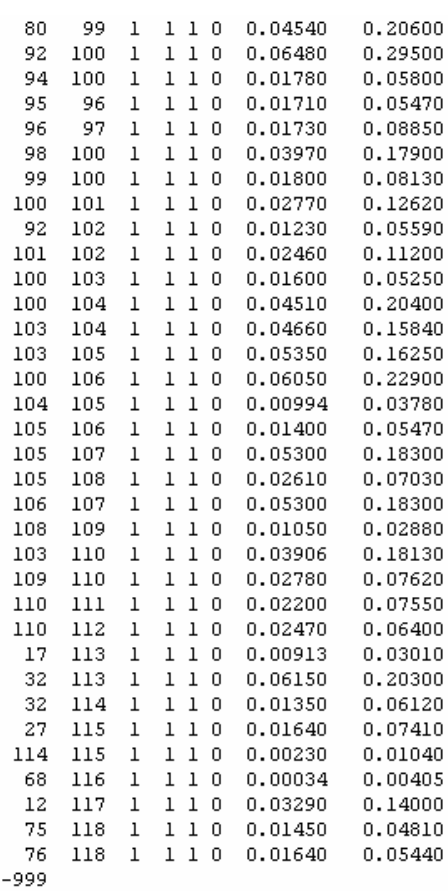

$\begin{array}{lllllll}0.05460 & 0 & 0 & 0 & 0 & 0 & 0.0 \\ 0.04720 & 0 & 0 & 0 & 0 & 0 & 0.0 \\ 0.06040 & 0 & 0 & 0 & 0 & 0 & 0.0 \\ 0.01474 & 0 & 0 & 0 & 0 & 0 & 0.0 \\ 0.02400 & 0 & 0 & 0 & 0 & 0 & 0.0 \\ 0.04760 & 0 & 0 & 0 & 0 & 0 & 0.0 \\ 0.02160 & 0 & 0 & 0 & 0 & 0 & 0.0 \\ 0.03280 & 0 & 0 & 0 & 0 & 0 & 0.0 \\ 0.01464 & 0 & 0 & 0 & 0 & 0 & 0.0 \\ 0.02940 & 0 & 0 & 0 & 0 & 0 & 0.0 \\ 0.05360 & 0 & 0 & 0 & 0 & 0 & 0.0 \\ 0.05410 & 0 & 0 & 0 & 0 & 0 & 0.0 \\ 0.04070 & 0 & 0 & 0 & 0 & 0 & 0.0 \\ 0.04080 & 0 & 0 & 0 & 0 & 0 & 0.0 \\ 0.06200 & 0 & 0 & 0 & 0 & 0 & 0.0 \\ 0.00986 & 0 & 0 & 0 & 0 & 0 & 0.0 \\ 0.01434 & 0 & 0 & 0 & 0 & 0 & 0.0 \\ 0.04720 & 0 & 0 & 0 & 0 & 0 & 0.0 \\ 0.01844 & 0 & 0 & 0 & 0 & 0 & 0.0 \\ 0.04720 & 0 & 0 & 0 & 0 & 0 & 0.0 \\ 0.00760 & 0 & 0 & 0 & 0 & 0 & 0.0 \\ 0.04610 & 0 & 0 & 0 & 0 & 0 & 0.0 \\ 0.02020 & 0 & 0 & 0 & 0 & 0 & 0.0 \\ 0.02000 & 0 & 0 & 0 & 0 & 0 & 0.0 \\ 0.06200 & 0 & 0 & 0 & 0 & 0 & 0.0 \\ 0.00768 & 0 & 0 & 0 & 0 & 0 & 0.0 \\ 0.05180 & 0 & 0 & 0 & 0 & 0 & 0.0 \\ 0.01628 & 0 & 0 & 0 & 0 & 0 & 0.0 \\ 0.01972 & 0 & 0 & 0 & 0 & 0 & 0.0 \\ 0.00276 & 0 & 0 & 0 & 0 & 0 & 0.0 \\ 0.16400 & 0 & 0 & 0 & 0 & 0 & 0.0 \\ 0.03580 & 0 & 0 & 0 & 0 & 0 & 0.0 \\ 0.01198 & 0 & 0 & 0 & 0 & 0 & 0.0 \\ 0.01356 & 0 & 0 & 0 & 0 & 0 & 0.0 \\ 0 & & & & & & \end{array}$

$\begin{array}{llllll}0.0 & 0.0 & 0.0 & 0.0 & 0.0 & 0.0 \\ 0.0 & 0.0 & 0.0 & 0.0 & 0.0 & 0.0 \\ 0.0 & 0.0 & 0.0 & 0.0 & 0.0 & 0.0 \\ 0.00 .0 & 0.0 & 0.0 & 0.0 & 0.0 \\ 0.0 & 0.0 & 0.0 & 0.0 & 0.0 & 0.0 \\ 0.0 & 0.0 & 0.0 & 0.0 & 0.0 & 0.0 \\ 0.0 & 0.0 & 0.0 & 0.0 & 0.0 & 0.0 \\ 0.0 & 0.0 & 0.0 & 0.0 & 0.0 & 0.0 \\ 0.0 & 0.0 & 0.0 & 0.0 & 0.0 & 0.0 \\ 0.0 & 0.0 & 0.0 & 0.0 & 0.0 & 0.0 \\ 0.0 & 0.0 & 0.0 & 0.0 & 0.0 & 0.0 \\ 0.0 & 0.0 & 0.0 & 0.0 & 0.0 & 0.0 \\ 0.0 & 0.0 & 0.0 & 0.0 & 0.0 & 0.0 \\ 0.0 & 0.0 & 0.0 & 0.0 & 0.0 & 0.0 \\ 0.0 & 0.0 & 0.0 & 0.0 & 0.0 & 0.0 \\ 0.0 & 0.0 & 0.0 & 0.0 & 0.0 & 0.0 \\ 0.0 & 0.0 & 0.0 & 0.0 & 0.0 & 0.0 \\ 0.0 & 0.0 & 0.0 & 0.0 & 0.0 & 0.0 \\ 0.0 & 0.0 & 0.0 & 0.0 & 0.0 & 0.0 \\ 0.0 & 0.0 & 0.0 & 0.0 & 0.0 & 0.0 \\ 0.0 & 0.0 & 0.0 & 0.0 & 0.0 & 0.0 \\ 0.0 & 0.0 & 0.0 & 0.0 & 0.0 & 0.0 \\ 0.0 & 0.0 & 0.0 & 0.0 & 0.0 & 0.0 \\ 0.0 & 0.0 & 0.0 & 0.0 & 0.0 & 0.0 \\ 0.0 & 0.0 & 0.0 & 0.0 & 0.0 & 0.0 \\ 0.0 & 0.0 & 0.0 & 0.0 & 0.0 & 0.0 \\ 0.0 & 0.0 & 0.0 & 0.0 & 0.0 & 0.0 \\ 0.0 & 0.0 & 0.0 & 0.0 & 0.0 & 0.0 \\ 0.0 & 0.0 & 0.0 & 0.0 & 0.0 & 0.0 \\ 0.0 & 0.0 & 0.0 & 0.0 & 0.0 & 0.0 \\ 0.0 & 0.0 & 0.0 & 0.0 & 0.0 & 0.0 \\ 0.0 & 0.0 & 0.0 & 0.0 & 0.0 & 0.0 \\ 0.0 & 0.0 & 0.0 & 0.0 & 0.0 & 0.0 \\ 0.0 & 0.0 & 0.0 & 0.0 & 0.0 & 0.0\end{array}$




\section{GLOSSÁRIO}

Variáveis de estado - as variáveis de estado de um sistema dinâmico são as grandezas cujo conjunto de valores determina o estado do sistema. Se forem necessárias, pelo menos, $n$ variáveis $x_{1}, x_{2}, x_{3}, \ldots, x_{n}$ para descrever completamente $\mathrm{o}$ comportamento de um sistema dinâmico (de modo que uma vez conhecidos os valores do sinal de entrada para $t \geq t_{0}$, o estado futuro do sistema esteja inteiramente determinado), tais $n$ variáveis formarão um conjunto de variáveis de estado.

Espaço de estados - Se um sistema possui três variáveis de estado, então o vetor de estados possui três componentes que podem ser visualizadas como um ponto em um espaço tridimensional. Ou seja, o espaço de estados é tridimensional. Este conceito é análogo para mais de três variáveis de estado, por exemplo, $n$ estados. Desta forma, o vetor de estados é representado por um ponto em um espaço de estados $n$ dimensional.

Equilíbrio - Equilíbrio de uma equação diferencial é um ponto, em um espaço de estados, que não se move. Se um estado está inicialmente em equilíbrio, assim deverá permanecer. Um equilíbrio estável para um modelo de sistema de potência corresponde a um ponto de operação deste mesmo sistema.

Sistema ortonormal - um sistema é ortonormal se os vetores que formam sua base são normalizados (têm comprimento igual a um) e mutuamente ortogonais. Um conjunto ortonormal de vetores é sempre linearmente independente. 